\title{
A Convenient and General Iron-Catalyzed Hydrosilylation of Aldehydes
}

\author{
Nadim S. Shaikh, Kathrin Junge, and Matthias Beller \\ Leibniz-Institut für Katalyse e.V. an der Universität Rostock, Albert-Einstein-Str. 29a, D- \\ 18059 Rostock, Germany \\ matthias.beller@catalysis.de
}

\section{Supporting Information-2}

Contents

Page

${ }^{1} \mathrm{H}$ NMR spectra of benzyl alcohol (1b).............................S4

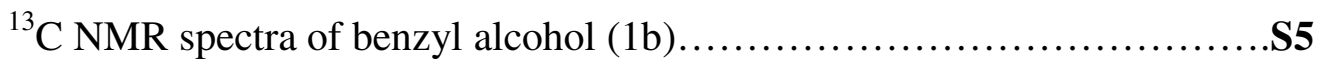

${ }^{1} \mathrm{H}$ NMR spectra of 4-tolyl-methanol (2b)...............................6

${ }^{13} \mathrm{C}$ NMR spectra of 4-tolyl-methanol (2b)..........................S7

${ }^{1} \mathrm{H}$ NMR spectra of 4-tert-butylphenyl-methanol (3b)...................S8

${ }^{13} \mathrm{C}$ NMR spectra of 4-tert-butylphenyl-methanol (3b)....................S9

${ }^{1} \mathrm{H}$ NMR spectra of 4-chlorophenyl-methanol (4b).....................S10

${ }^{13} \mathrm{C}$ NMR spectra of 4-chlorophenyl-methanol (4b)......................S11

${ }^{1} \mathrm{H}$ NMR spectra of 4-bromophenyl-methanol (5b).........................

${ }^{13} \mathrm{C}$ NMR spectra of 4-bromophenyl-methanol (5b)....................S13

${ }^{1} \mathrm{H}$ NMR spectra of 4-fluorophenyl-methanol (6b).......................S14

${ }^{13} \mathrm{C}$ NMR spectra of 4-fluorophenyl-methanol (6b)......................S15

${ }^{1} \mathrm{H}$ NMR spectra of 4-nitrophenyl-methanol (7b).......................S16

${ }^{13} \mathrm{C}$ NMR spectra of 4-nitrophenyl-methanol (7b).......................S17

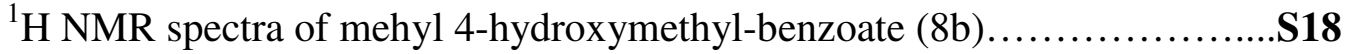

${ }^{13} \mathrm{C}$ NMR spectra of mehyl 4-hydroxymethyl-benzoate (8b).................S19

${ }^{1} \mathrm{H}$ NMR spectra of 4-methoxyphenyl-methanol (9b)....................S20

${ }^{13} \mathrm{C}$ NMR spectra of 4-methoxyphenyl-methanol (9b)....................S21

${ }^{1}$ H NMR spectra of 4-benzyloxyphenyl-methanol (10b)..................S22

${ }^{13} \mathrm{C}$ NMR spectra of 4-benzyloxyphenyl-methanol (10b).................S23

${ }^{1}$ H NMR spectra of (4-dimethylamino)phenyl-methanol (11b).............S24 
${ }^{13} \mathrm{C}$ NMR spectra of (4-dimethylamino)phenyl-methanol (11b).............S25

${ }^{1} \mathrm{H}$ NMR spectra of 4-methylsulphanylphenyl-methanol (12b).............S26

${ }^{13} \mathrm{C}$ NMR spectra of 4-methylsulphanylphenyl-methanol (12b)............S27

${ }^{1} \mathrm{H}$ NMR spectra of 3-hydroxymethyl-benzonitrile (13b).................S28

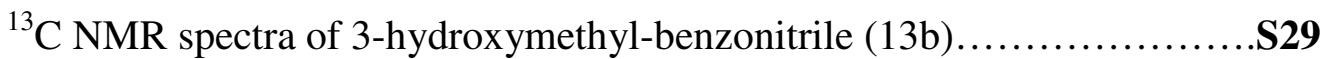

${ }^{1} \mathrm{H}$ NMR spectra of 3-fluorophenyl-methanol (14b).....................S30

${ }^{13} \mathrm{C}$ NMR spectra of 3-fluorophenyl-methanol (14b)....................S31

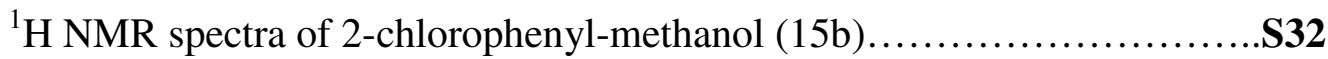

${ }^{13} \mathrm{C}$ NMR spectra of 2-chlorophenyl-methanol (15b)...................S33

${ }^{1} \mathrm{H}$ NMR spectra of biphenyl-2-yl-methanol (16b)......................S34

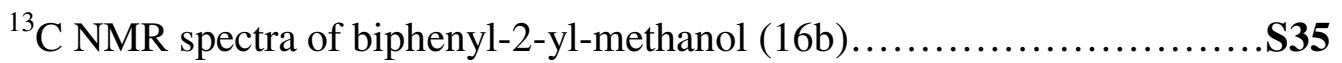

${ }^{1}$ H NMR spectra of 2-fluorophenyl-methanol (17b) .......................S36

${ }^{13} \mathrm{C}$ NMR spectra of 2-fluorophenyl-methanol (17b)....................S37

${ }^{1}$ H NMR spectra of 2,4,6-trimethylphenyl-methanol (18b)................S38

${ }^{13} \mathrm{C}$ NMR spectra of 2,4,6-trimethylphenyl-methanol (18b).................S39

${ }^{1}$ H NMR spectra of naphthalen-2-yl-methanol (19b)....................S40

${ }^{13} \mathrm{C}$ NMR spectra of naphthalen-2-yl-methanol (19b).....................S41

${ }^{1} \mathrm{H}$ NMR spectra of (3a,7a-dihydrobenzo[1,3]dioxol-5-yl)-methanol (20b)...S42

${ }^{13} \mathrm{C}$ NMR spectra of (3a,7a-dihydrobenzo[1,3]dioxol-5-yl)-methanol (20b)..S43

${ }^{1}$ H NMR spectra of phenanthren-9-yl-methanol (21b)....................S44

${ }^{13} \mathrm{C}$ NMR spectra of phenanthren-9-yl-methanol (21b)....................S45

${ }^{1}$ H NMR spectra of 1-phenylethanol (22b)................................646

${ }^{13} \mathrm{C}$ NMR spectra of 1-phenylethanol (22b)..........................S47

${ }^{1} \mathrm{H}$ NMR spectra of pyridin-4-yl-methanol (24b)......................S48

${ }^{13} \mathrm{C}$ NMR spectra of pyridin-4-yl-methanol (24b)........................S49

${ }^{1} \mathrm{H}$ NMR spectra of pyridin-3-yl-methanol (25b).......................S50

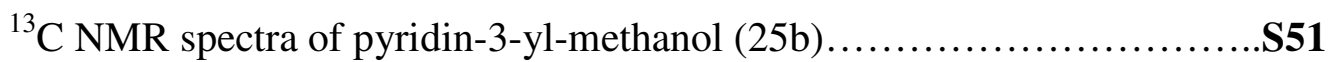

${ }^{1} \mathrm{H}$ NMR spectra of quinolin-3-yl-methanol (26b).........................552

${ }^{13} \mathrm{C}$ NMR spectra of quinolin-3-yl-methanol (26b).....................553

${ }^{1}$ H NMR spectra of furan-3-yl-methanol (27b)..........................554

${ }^{13} \mathrm{C}$ NMR spectra of furan-3-yl-methanol (27b)...........................555 
${ }^{1} \mathrm{H}$ NMR spectra of thiophen-2-yl-methanol (28b).......................S56

${ }^{13} \mathrm{C}$ NMR spectra of thiophen-2-yl-methanol (28b) ......................557

${ }^{1}$ H NMR spectra of cyclohexyl-methanol (29b).......................S58

${ }^{13} \mathrm{C}$ NMR spectra of cyclohexyl-methanol (29b)........................S59

${ }^{1} \mathrm{H}$ NMR spectra of 3-phenyl-propan-1-ol (30b).........................560

${ }^{13} \mathrm{C}$ NMR spectra of 3-phenyl-propan-1-ol (30b).........................S61

${ }^{1} \mathrm{H}$ NMR spectra of 1 -octanol (31b).................................62

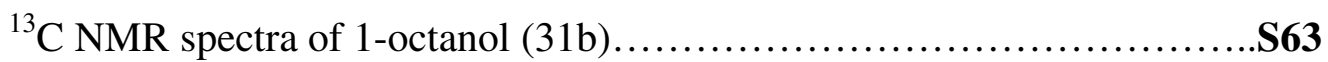

${ }^{1}$ H NMR spectra of 2,2-dimethyl-1-propanol (32b).......................S64

${ }^{13} \mathrm{C}$ NMR spectra of 2,2-dimethyl-1-propanol (32b).....................65

${ }^{1} \mathrm{H}$ NMR spectra of cyclohex-3-enyl-methanol (33b)....................566

${ }^{13} \mathrm{C}$ NMR spectra of cyclohex-3-enyl-methanol (33b)....................667

${ }^{1} \mathrm{H}$ NMR spectra of (2,2-dimethyl-[1,3]dioxolan-4-yl)-methanol (34b)......S68

${ }^{13} \mathrm{C}$ NMR spectra of (2,2-dimethyl-[1,3]dioxolan-4-yl)-methanol (34b)......S69

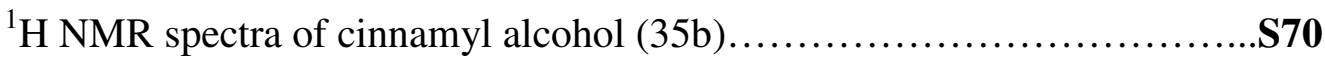

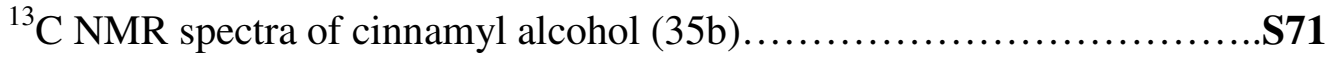


$070727.331 \quad 10 \quad 1 \quad$ D: 0707

Shaik, NS 2185

AulH CDC13/opt/topspin 070731

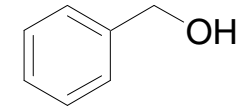

$1 b$

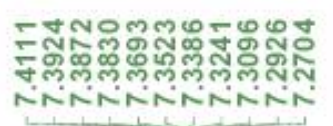

ninntinin
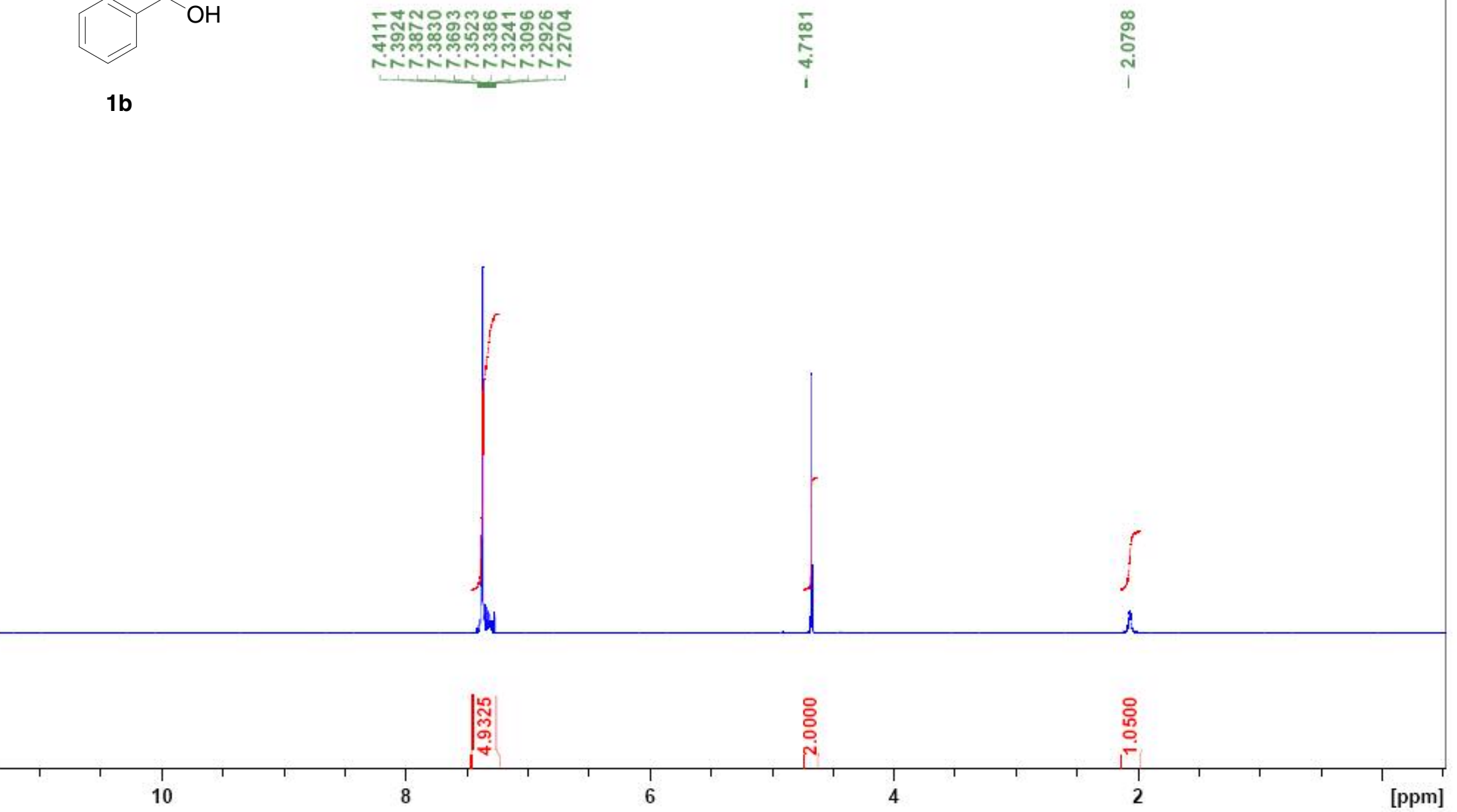


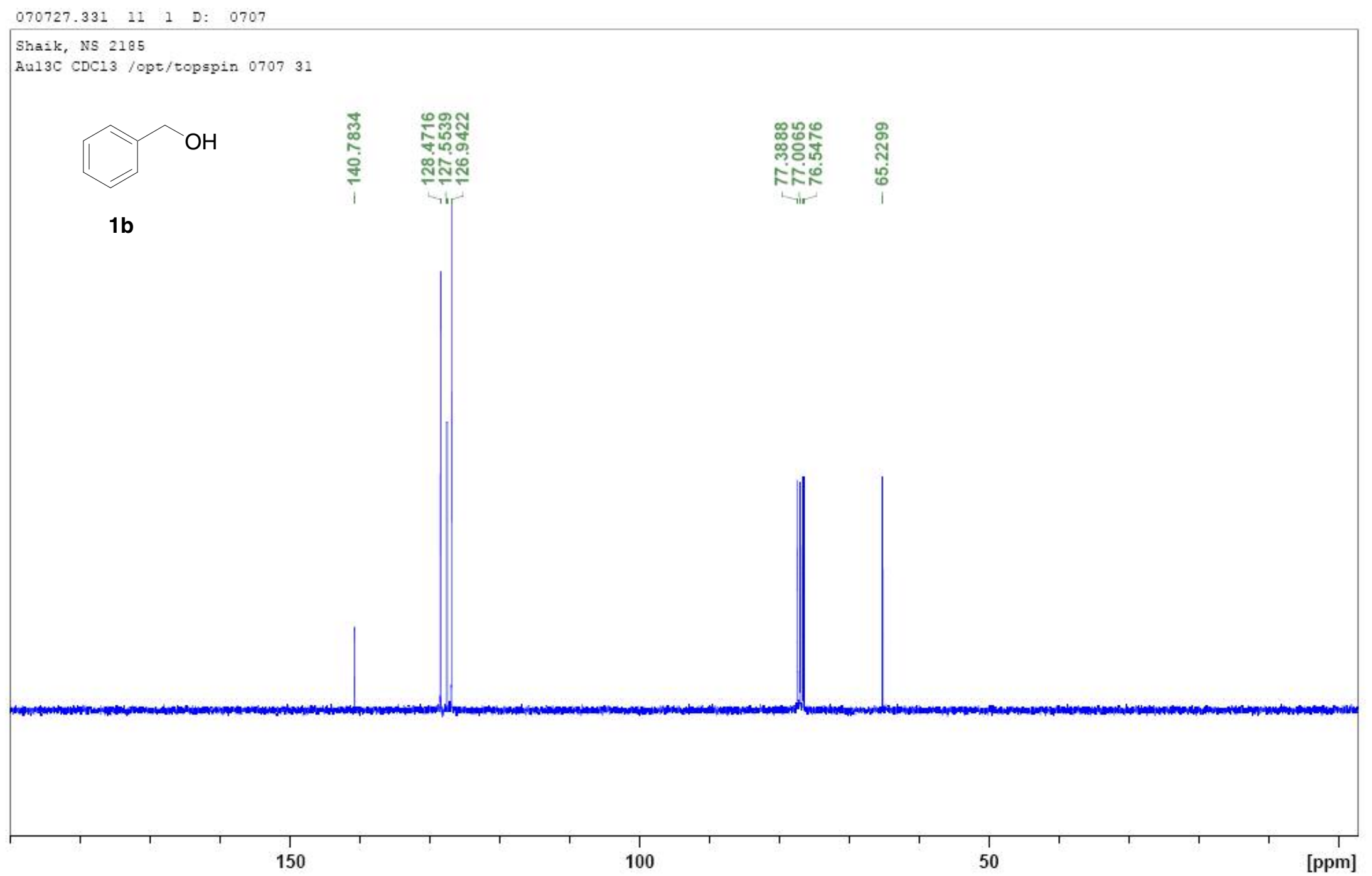




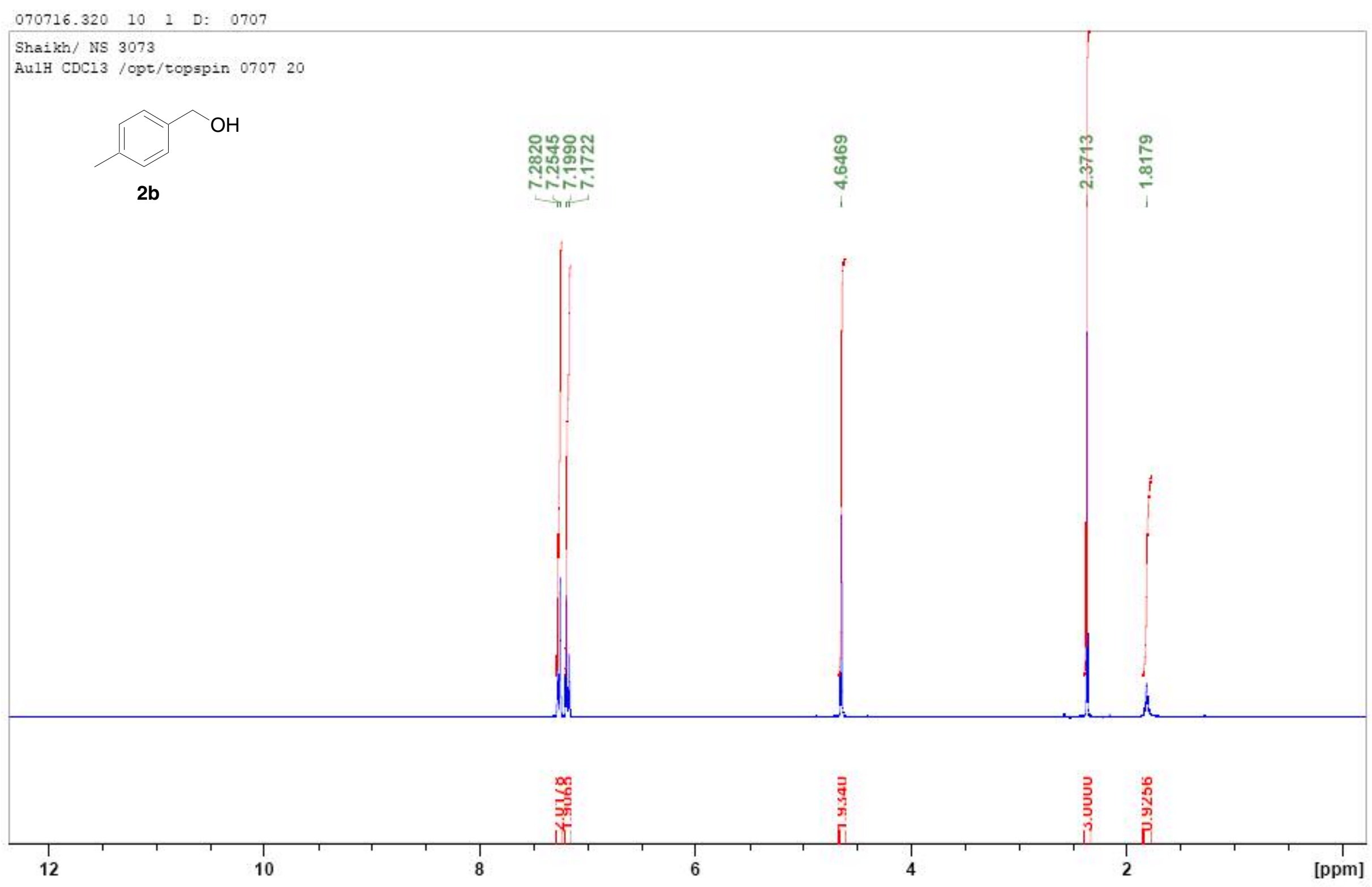


$070716.320 \quad 11 \quad 1 \quad$ D: 0707

Shaikh/ NS 3073

Au13C CDC13 /opt/topspin 070720

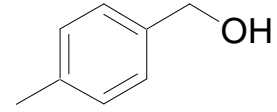

$2 b$

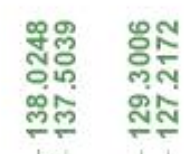

ᄂ

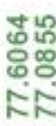

पे
ల్
iे

มฺุ

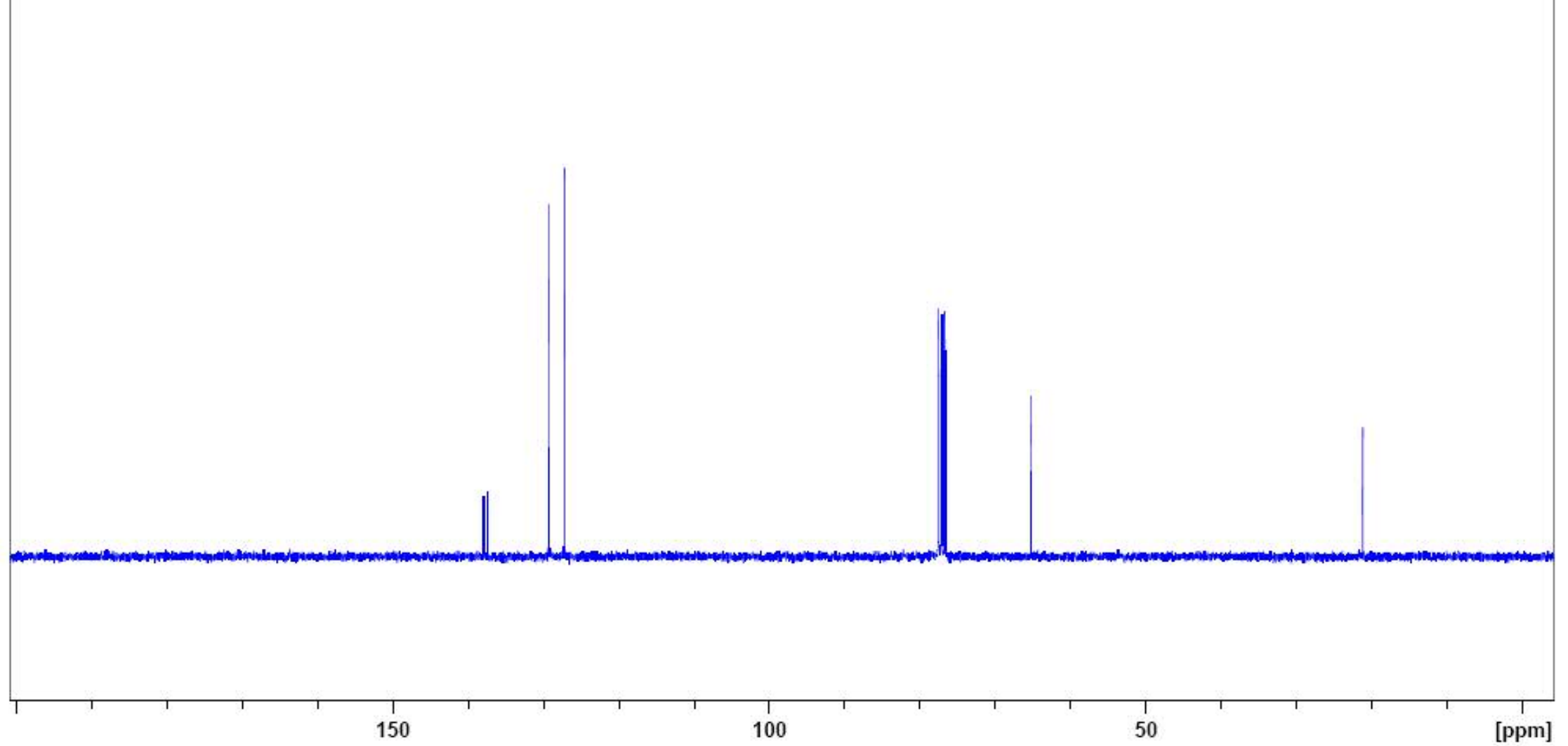


$070718.332 \quad 10 \quad 1 \quad$ D: 0707

Shaikh, NS 3103

AulH $\mathrm{CDCl}_{3}$ /opt/topspin 070732
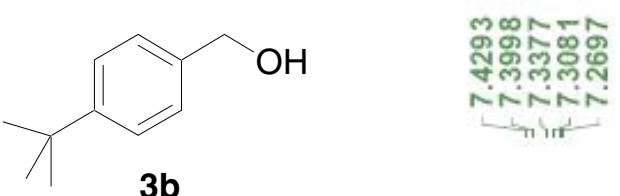

占

$3 b$
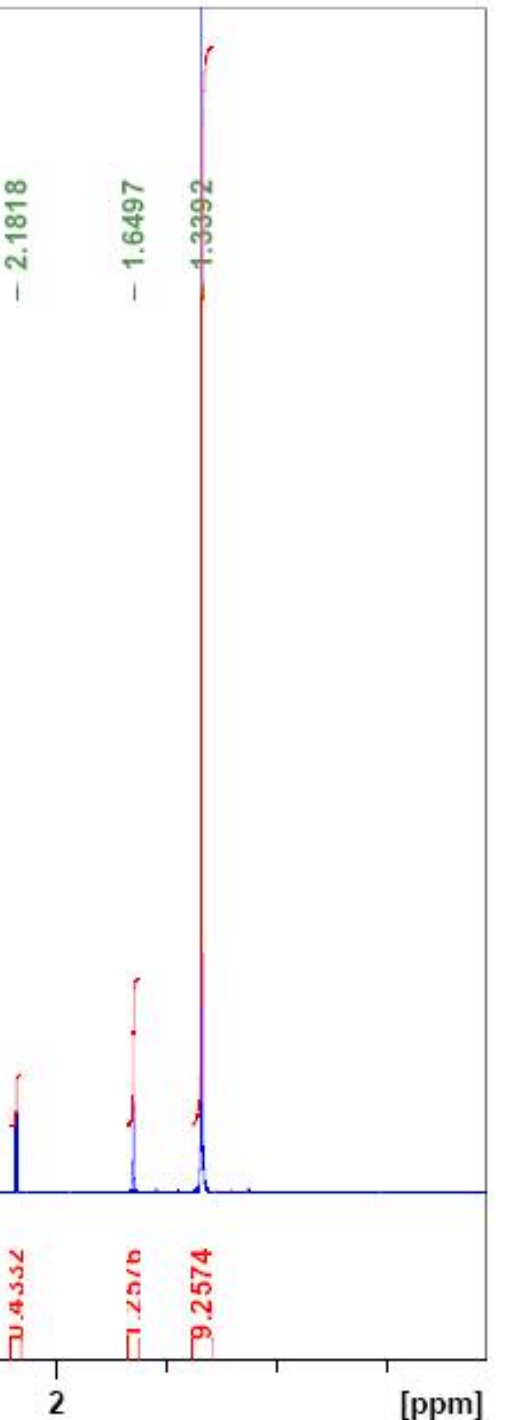


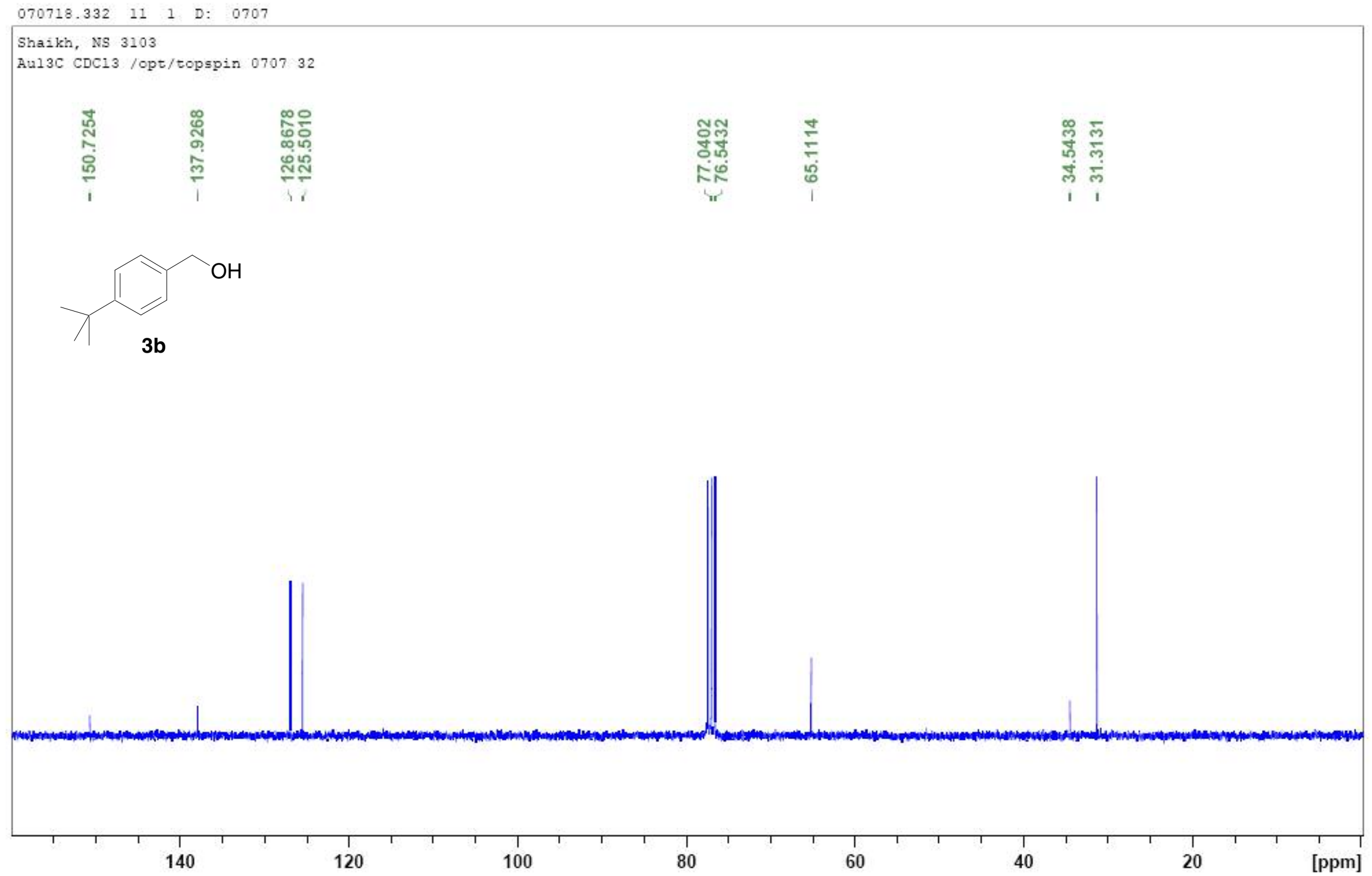




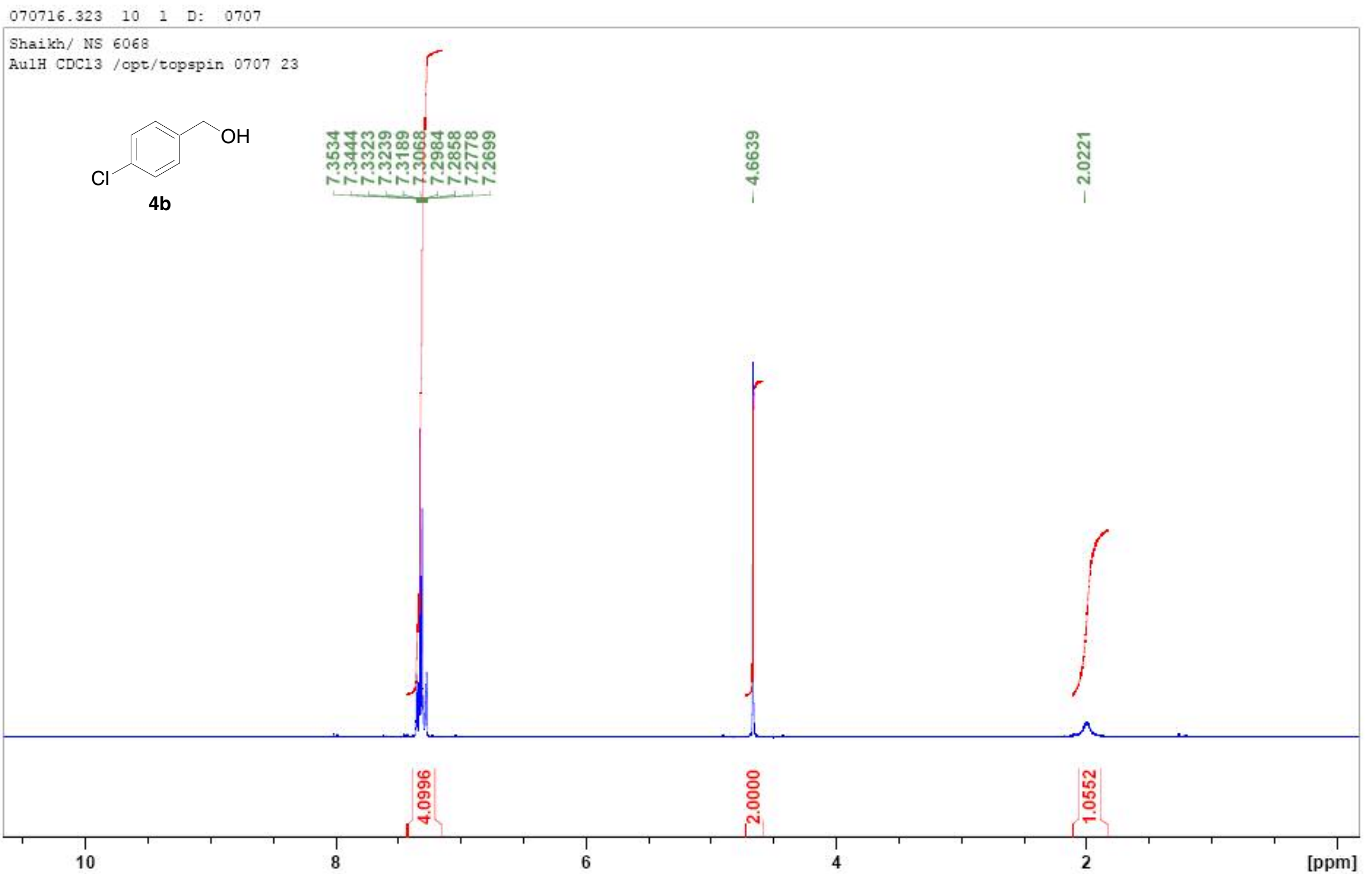




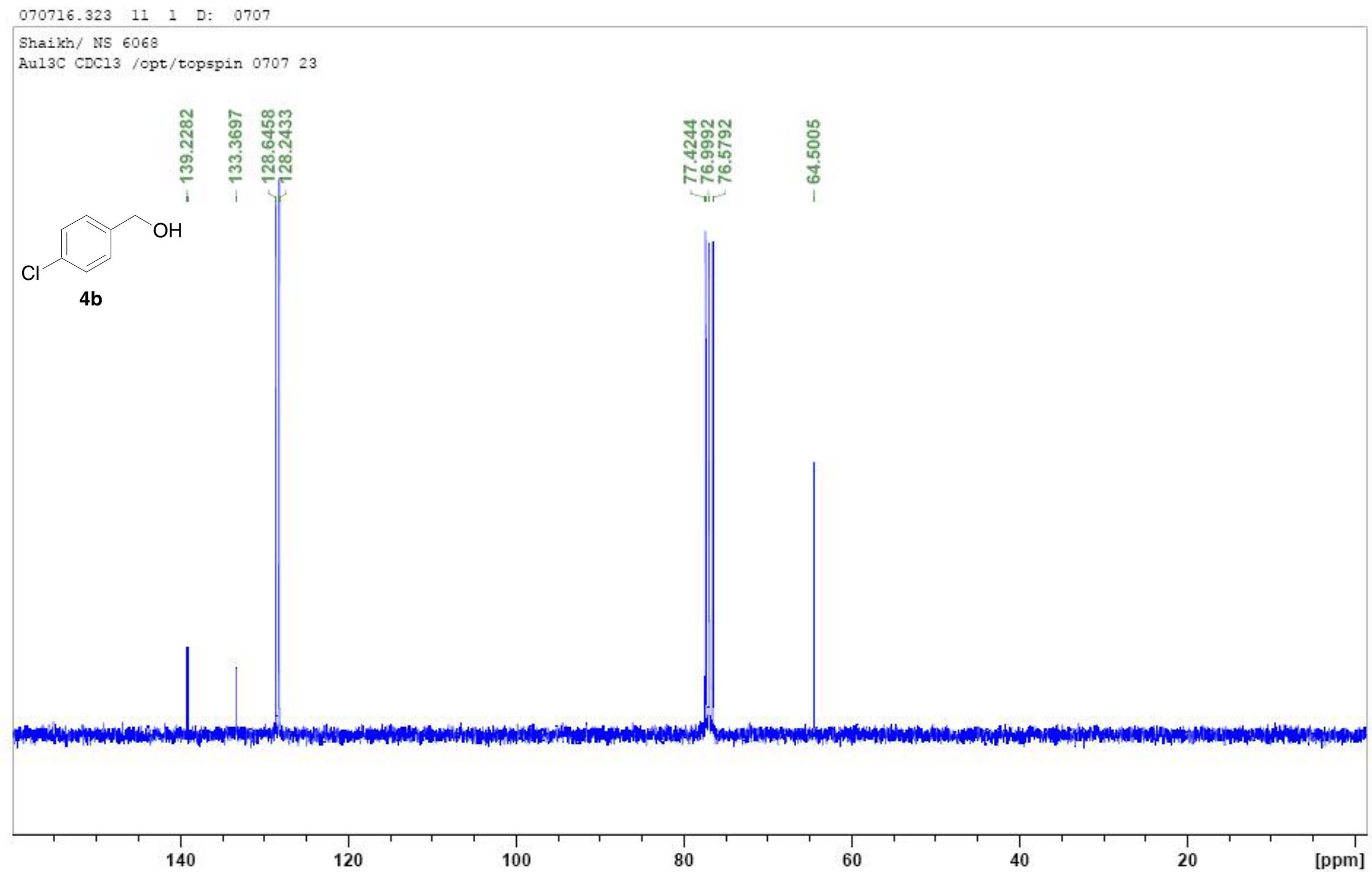




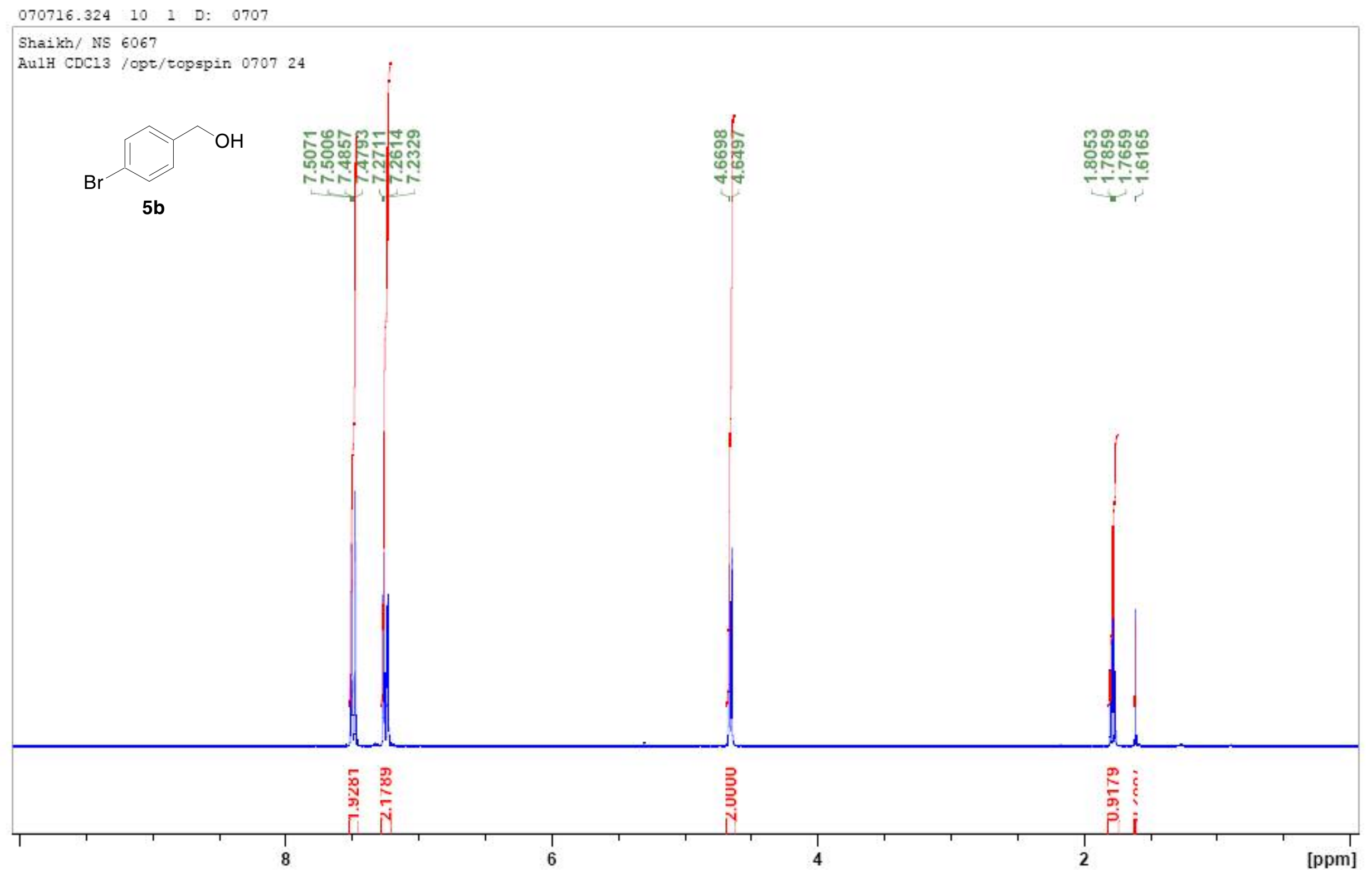


$070716.324 \quad 11 \quad 1 \quad$ D: 0707

Shaikh/ NS 6067

Au13C CDC13/opt/topspin 070724

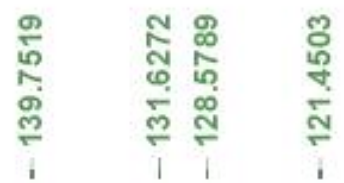

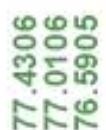

ก

$5 b$

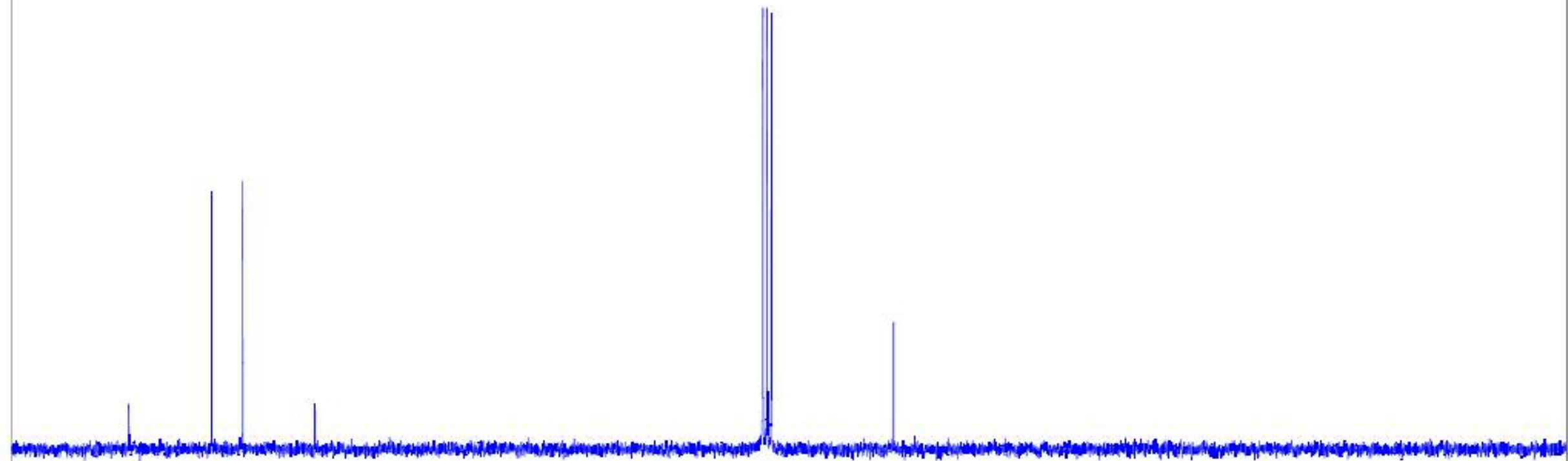

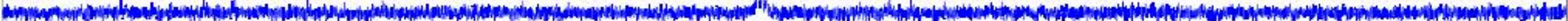

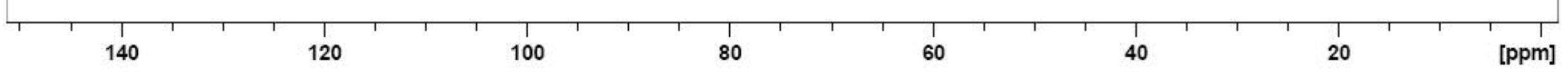




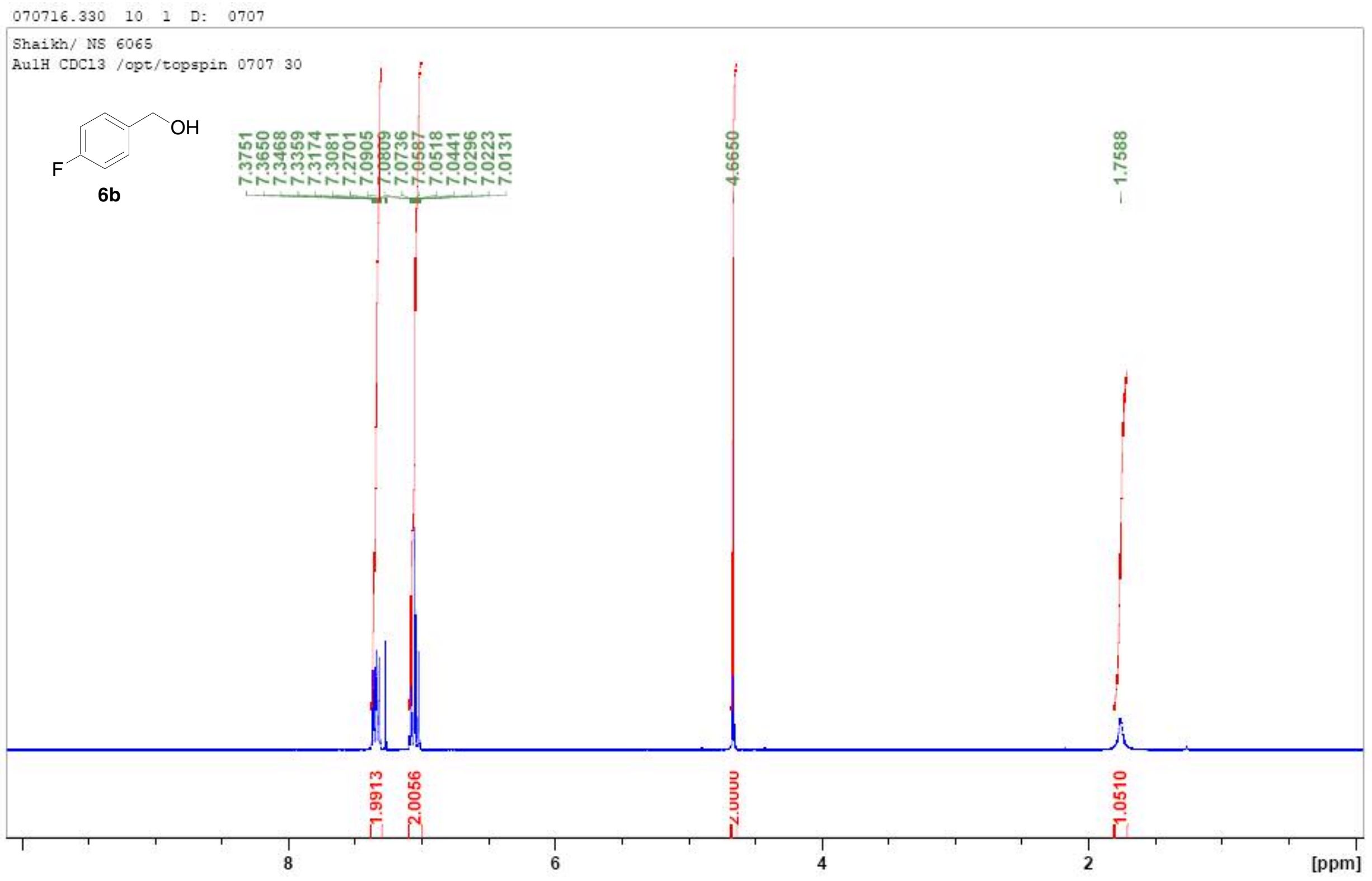




\section{$070716.330 \quad 11$ I D: 0707}

Shaikh/ NS 6065

Au13C CDC13/opt/topspin 070730

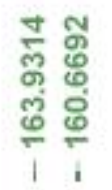

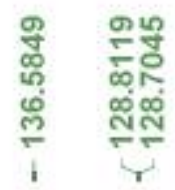

돓
ํํำ
ㄷำ

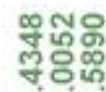

NRE

g
+⿱
广

$\mathrm{OH}_{\mathrm{OH}}$

$6 b$

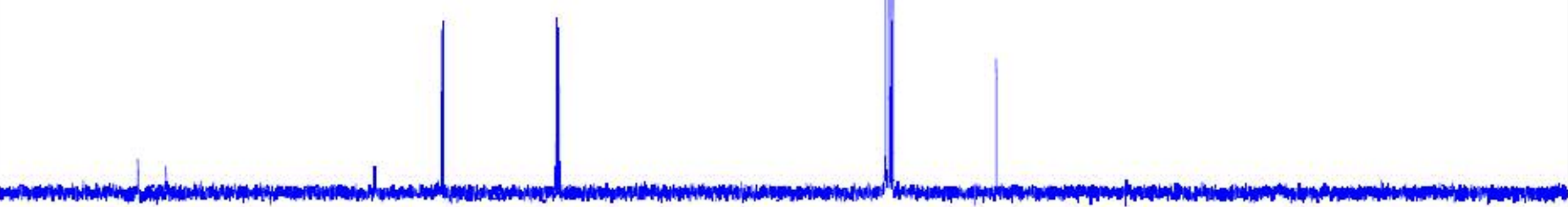


Supporting information-2

$070716.326 \quad 10 \quad 1 \quad$ D: 0707

Shaikh/NS 6066

AulH CDC13 /opt/topspin 070726
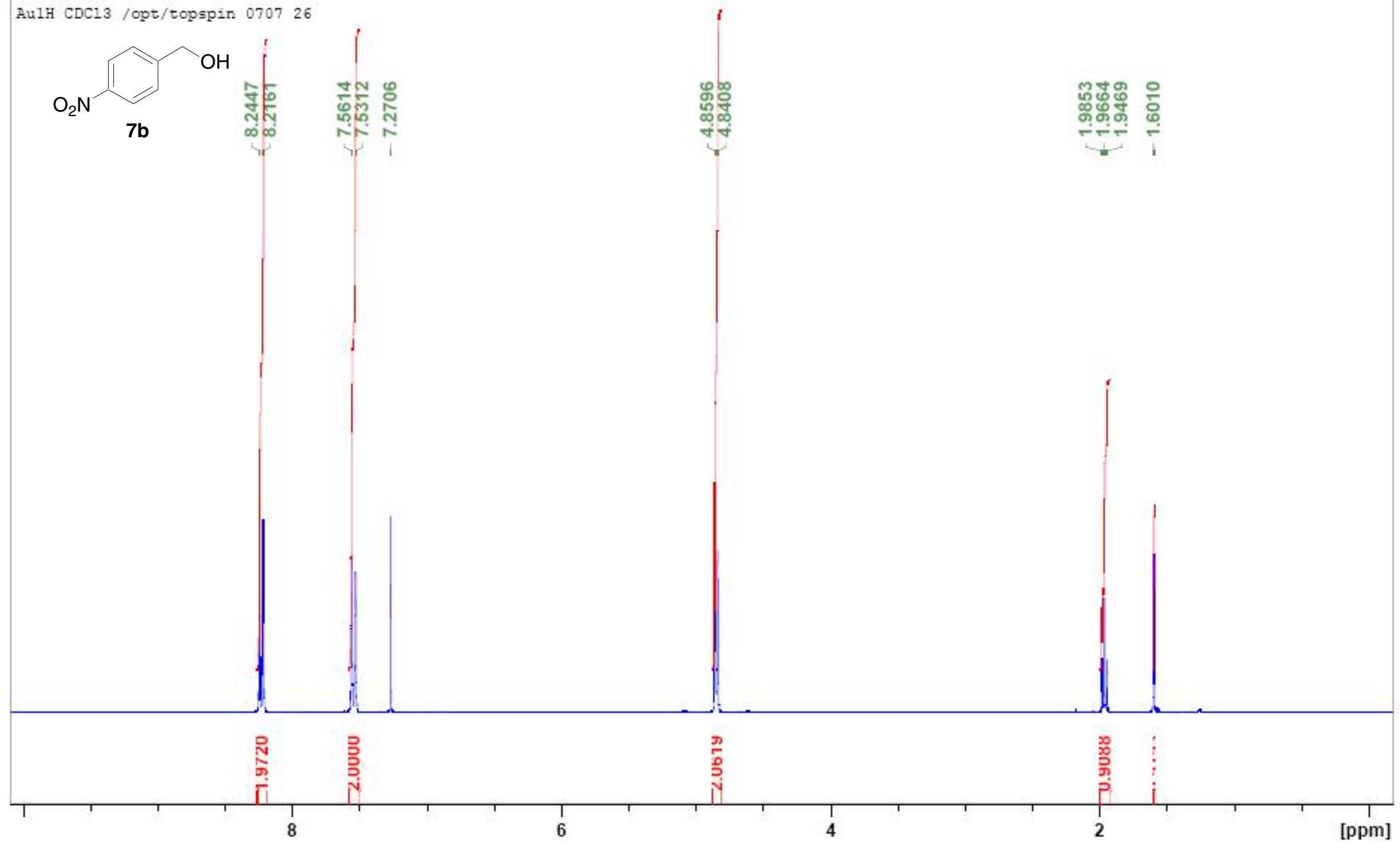
$\begin{array}{lllll}070716.326 & 11 & 1 & \mathrm{D}: & 0707\end{array}$

Shaikh/ NS 6066

Aul3C CDC13/opt/topspin 0707 26
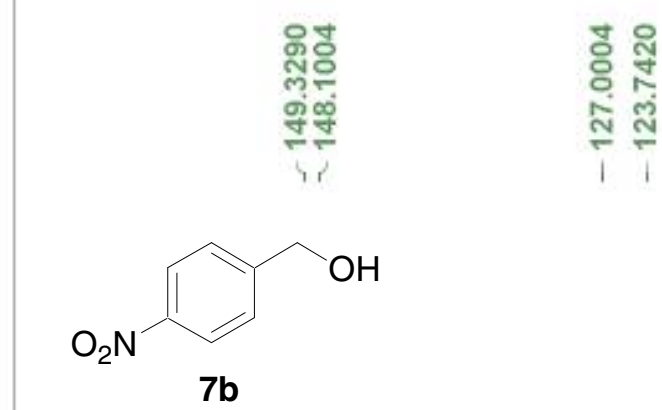

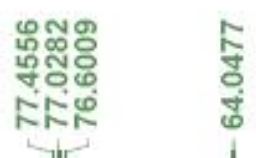

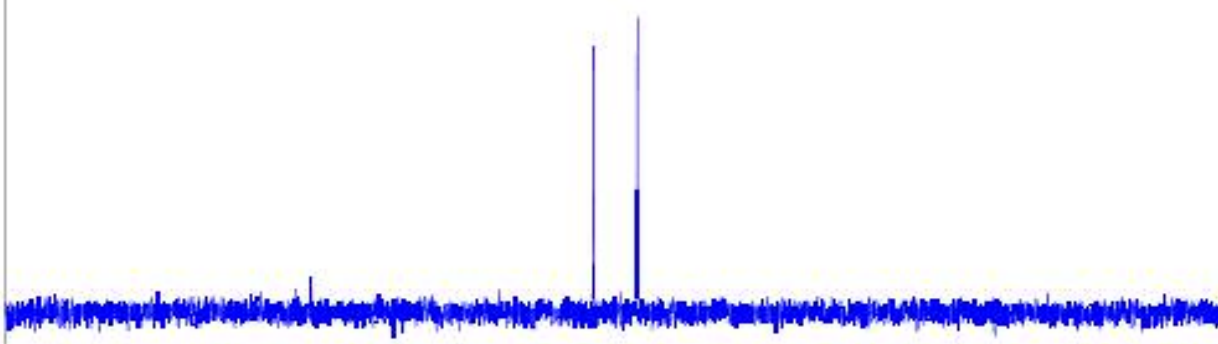

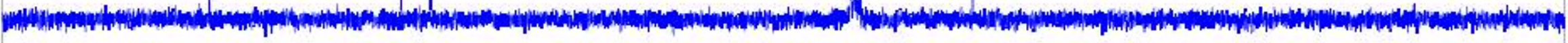

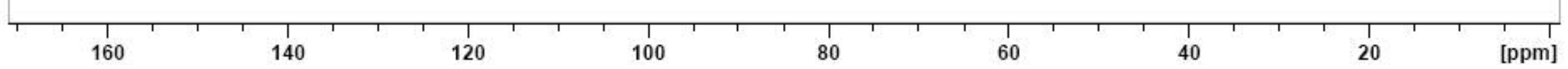


$070716.321 \quad 10 \quad 1 \quad$ D: 0707

Shaikh/NS 3087

AulH CDC13/opt/topspin 070721

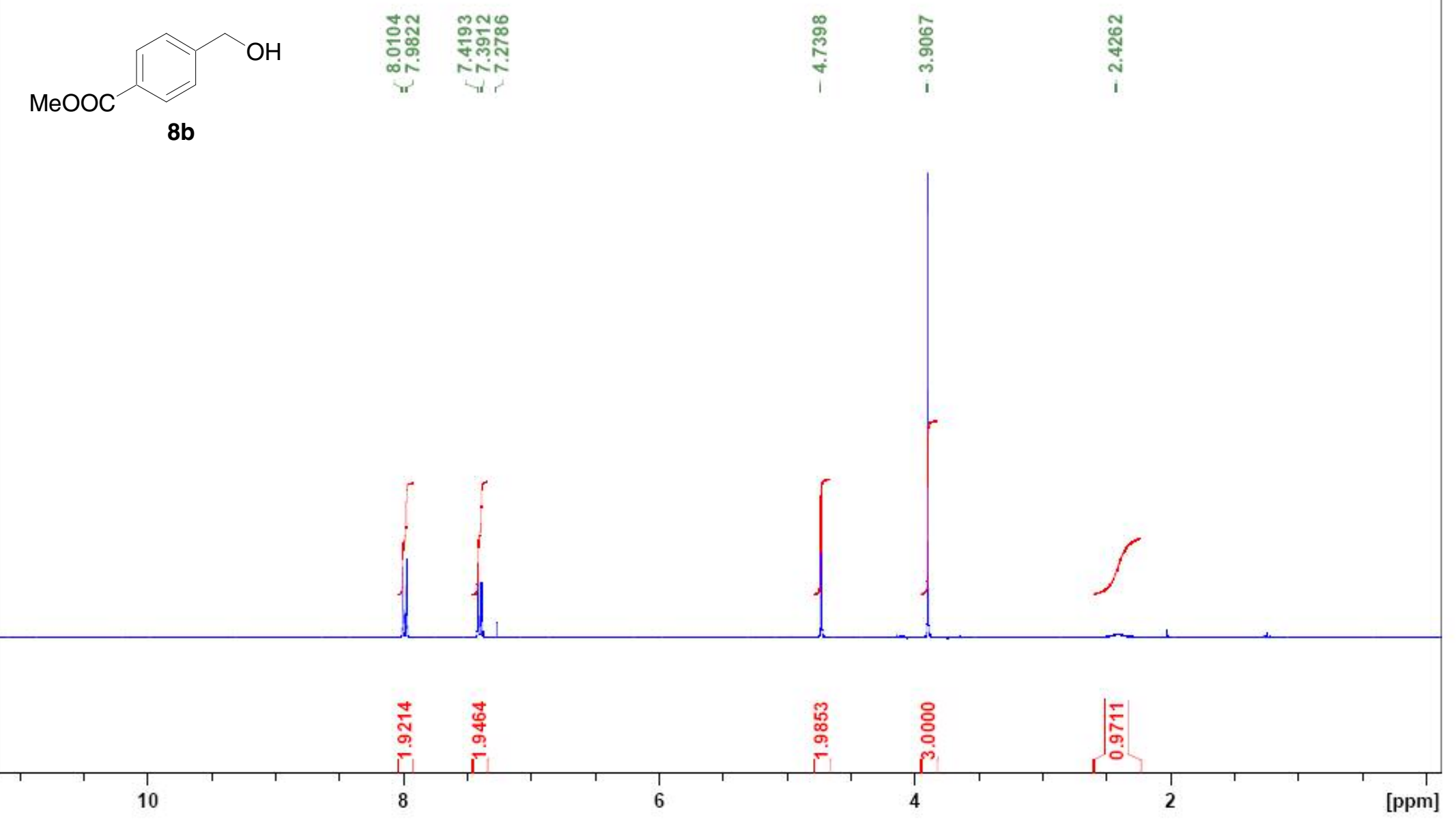


$\begin{array}{lllll}070716.321 & 11 & 1 & \text { D: } & 0707\end{array}$

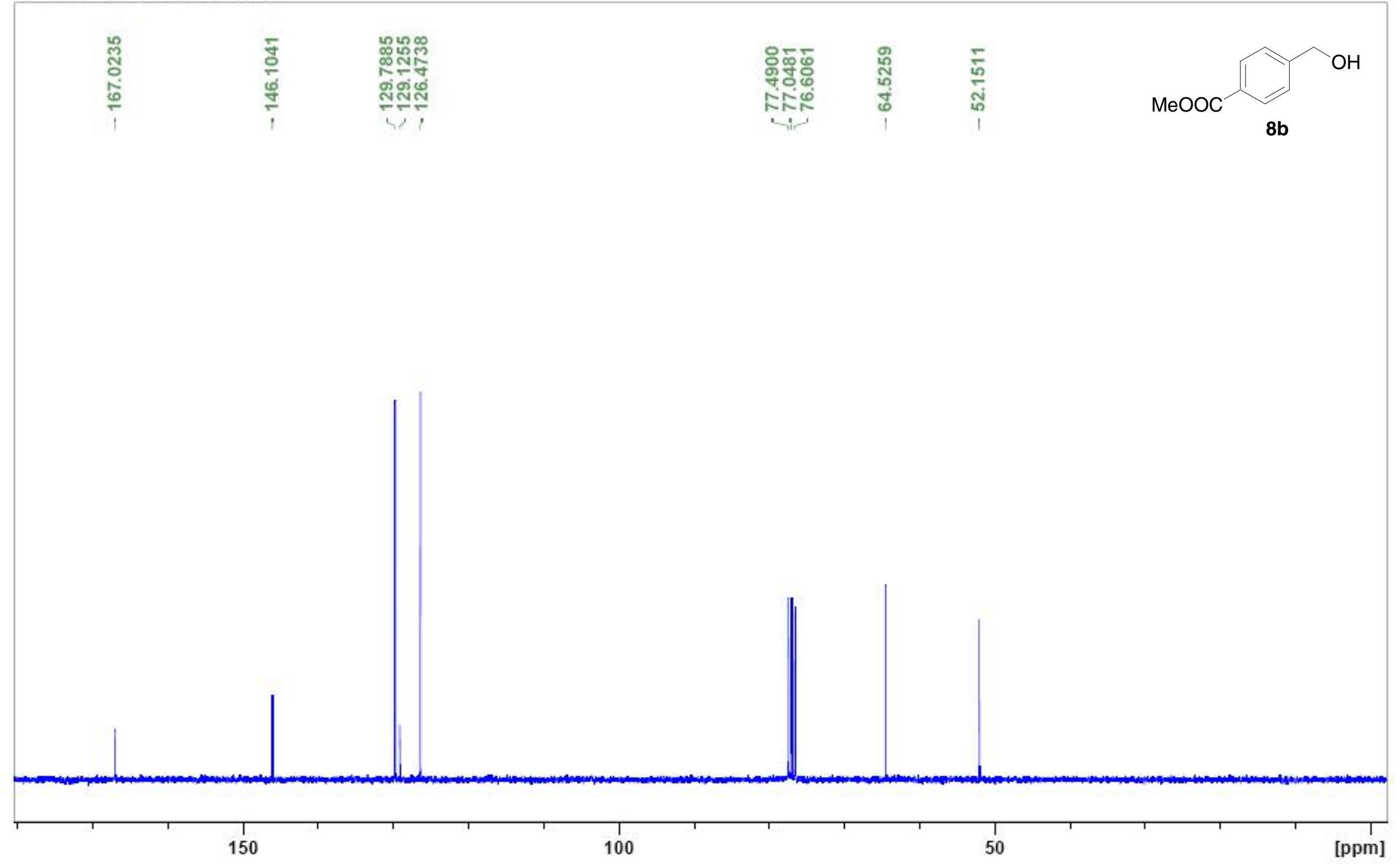


Supporting information-2

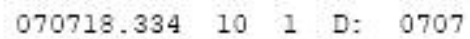

Shaikh, NS 3093

AulH CDC13 /opt/topspin 070734

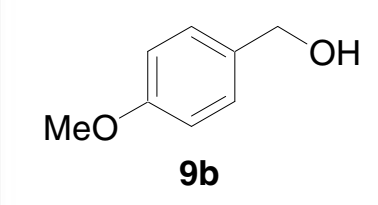

$\mathrm{OH}$

$9 b$

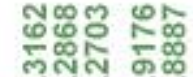

Mก๊

ir

$i$

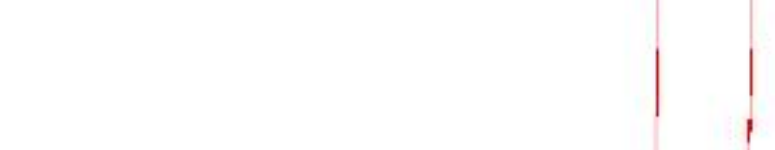
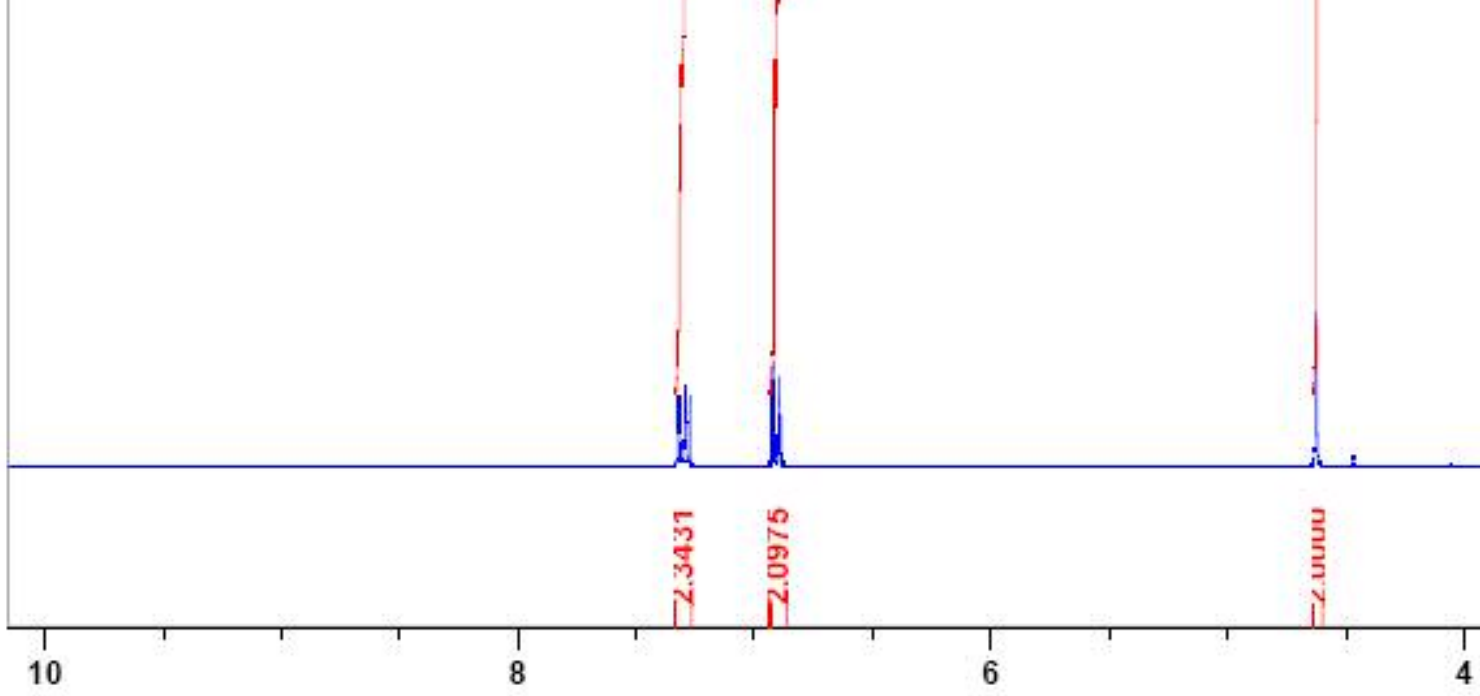

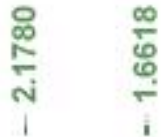

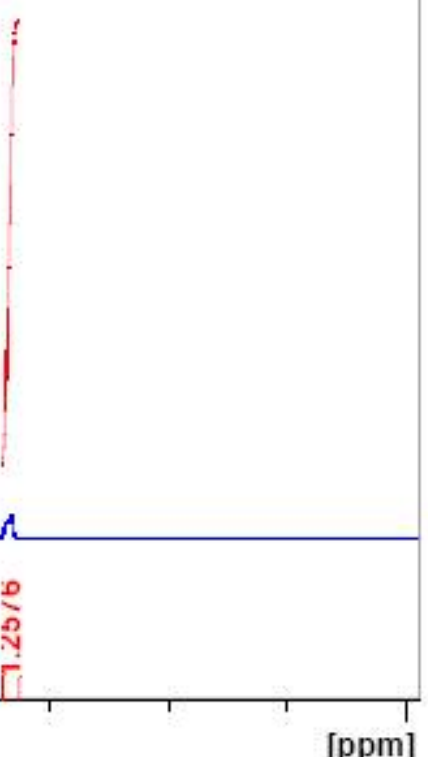


$070718.334 \quad 11 \quad 1 \quad$ D: 0707

Shaikh, NS 3093

Au13C CDC13/opt/topspin 070734

훙

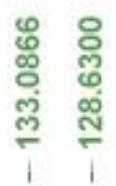

के
ले
ले

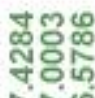 \\ NNE}

$\mathrm{MeO}$

$9 b$

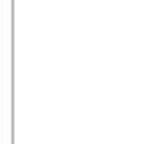

$+$

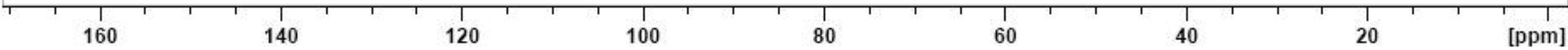


$070727.334 \quad 10 \quad 1 \quad$ D: $\quad 0707$

Shaik, NS 3110

AulH CDCl3/opt/topspin 070734

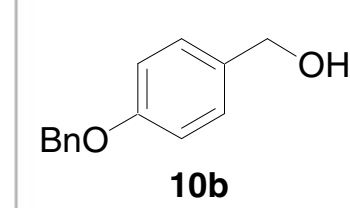

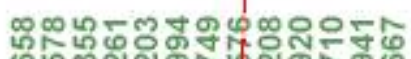

पy?

NNANRNANROE

$10 b$

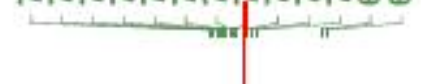

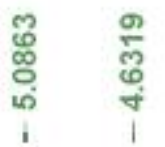

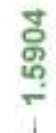
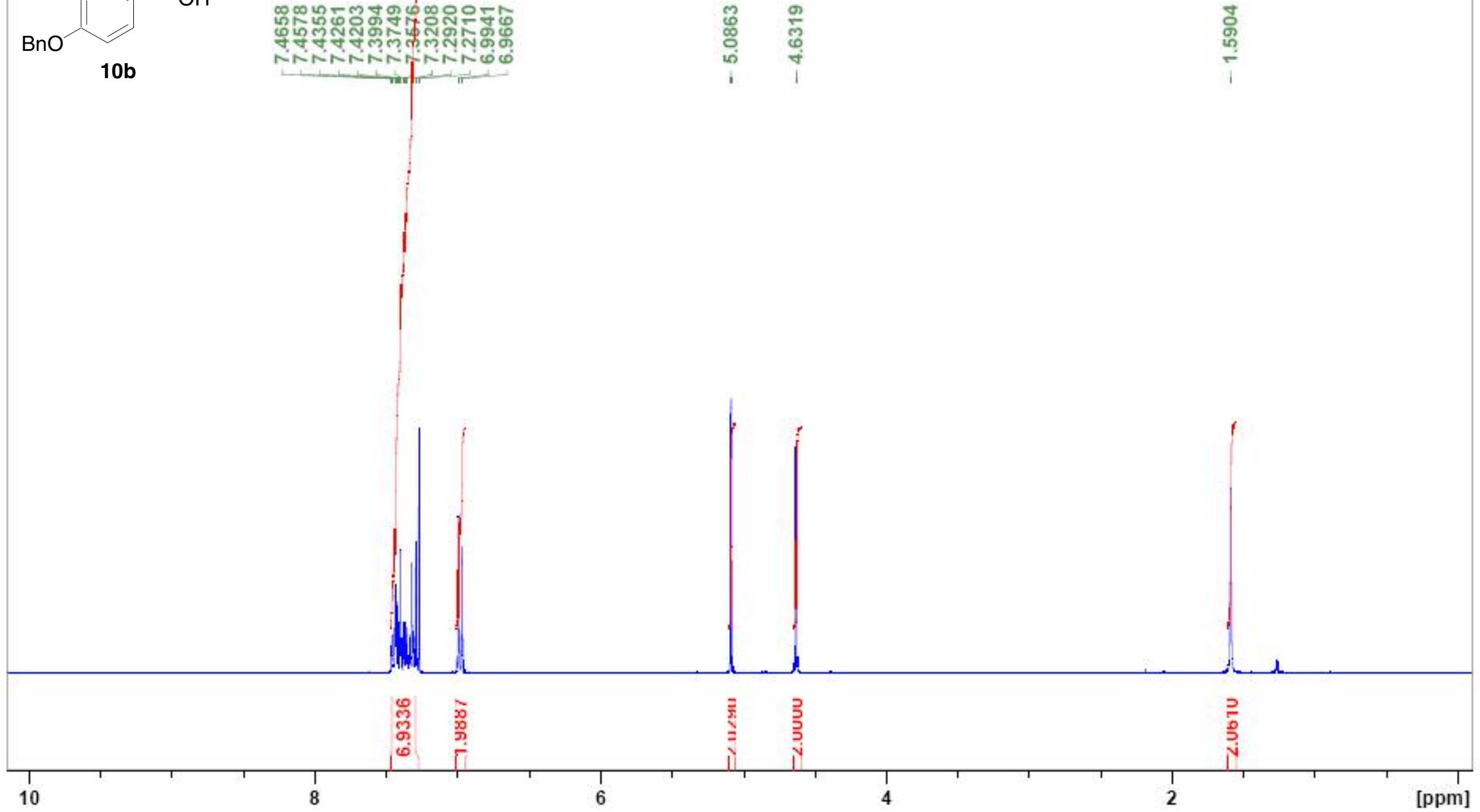


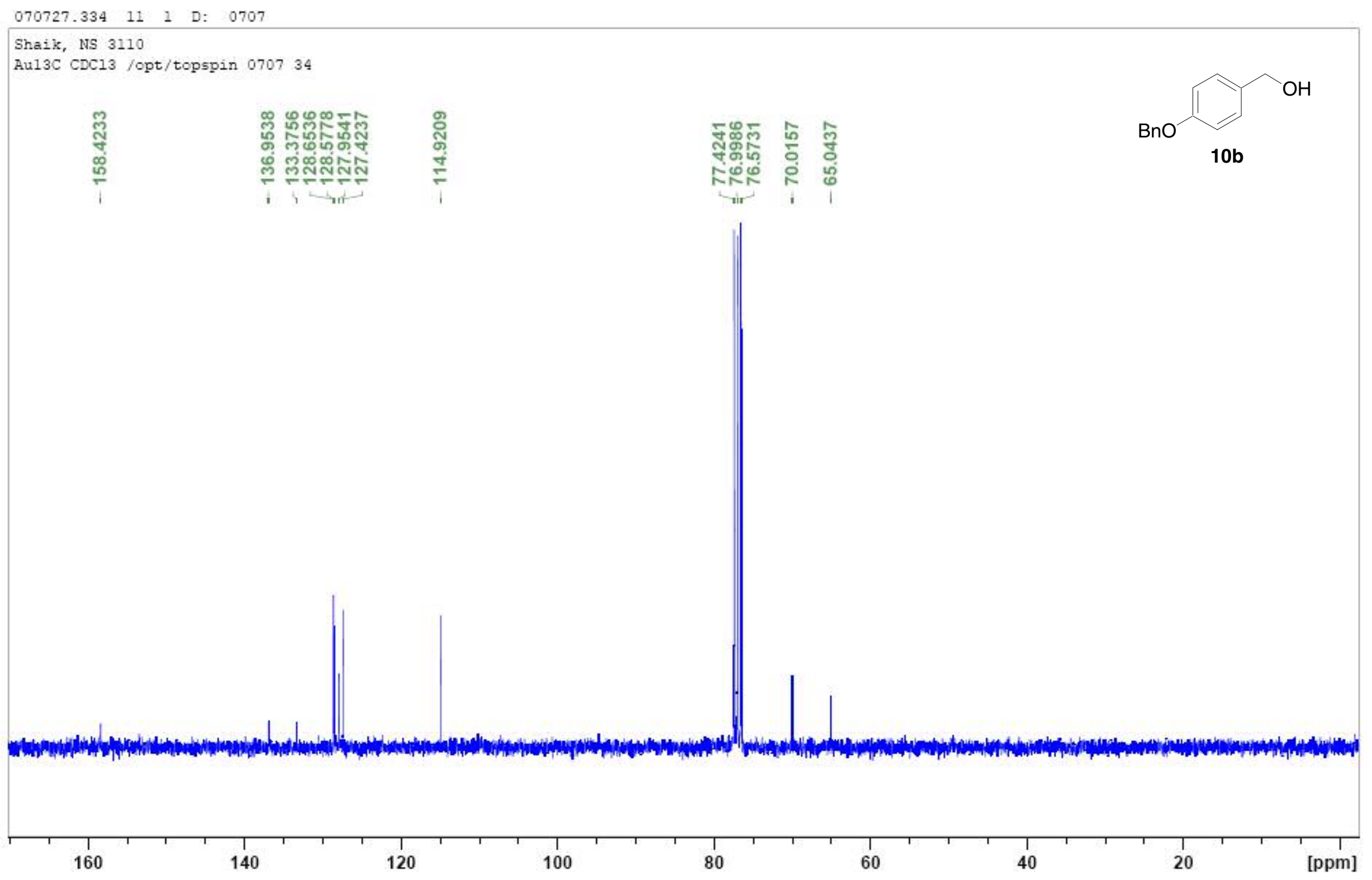


Supporting information-2

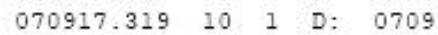

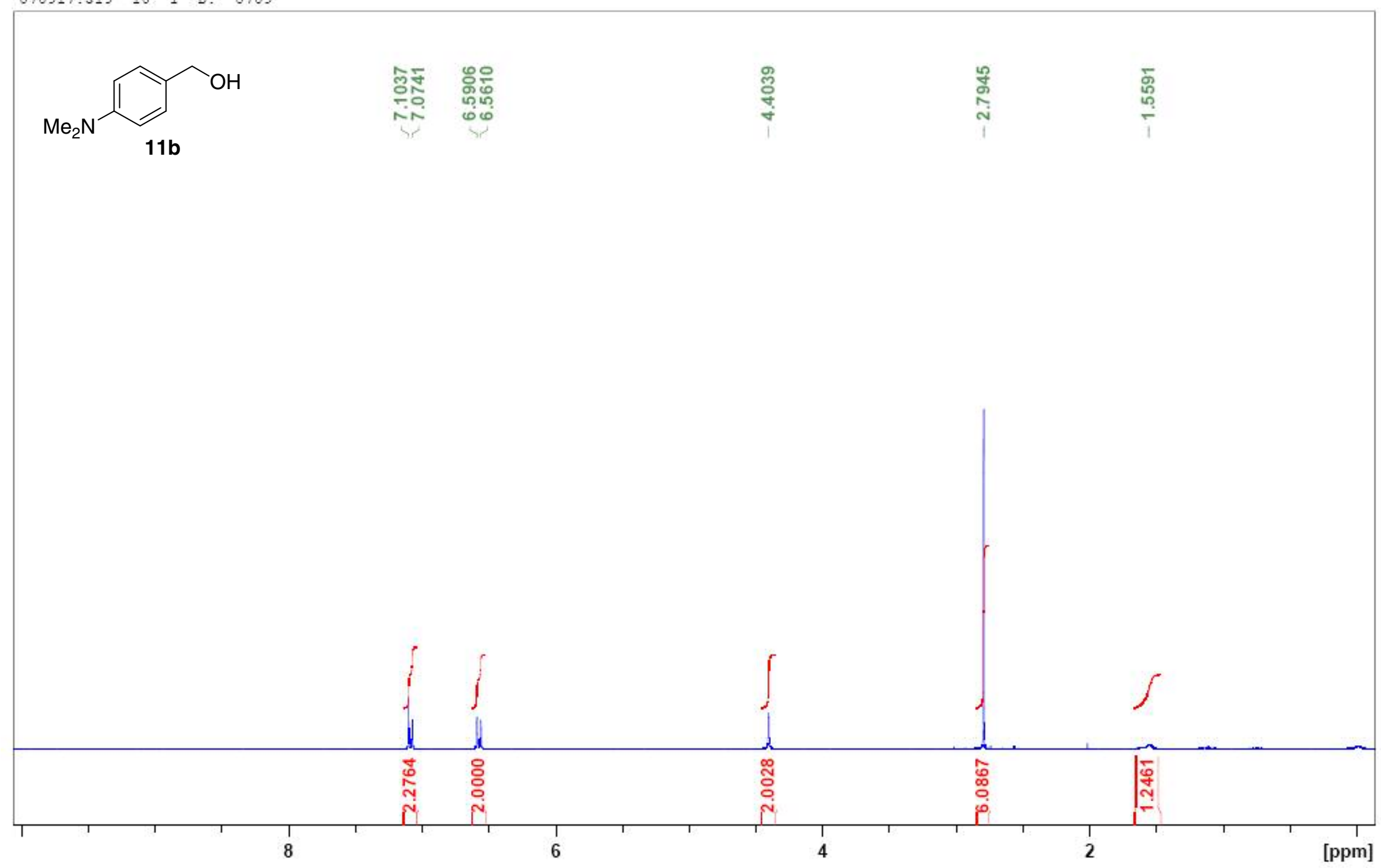




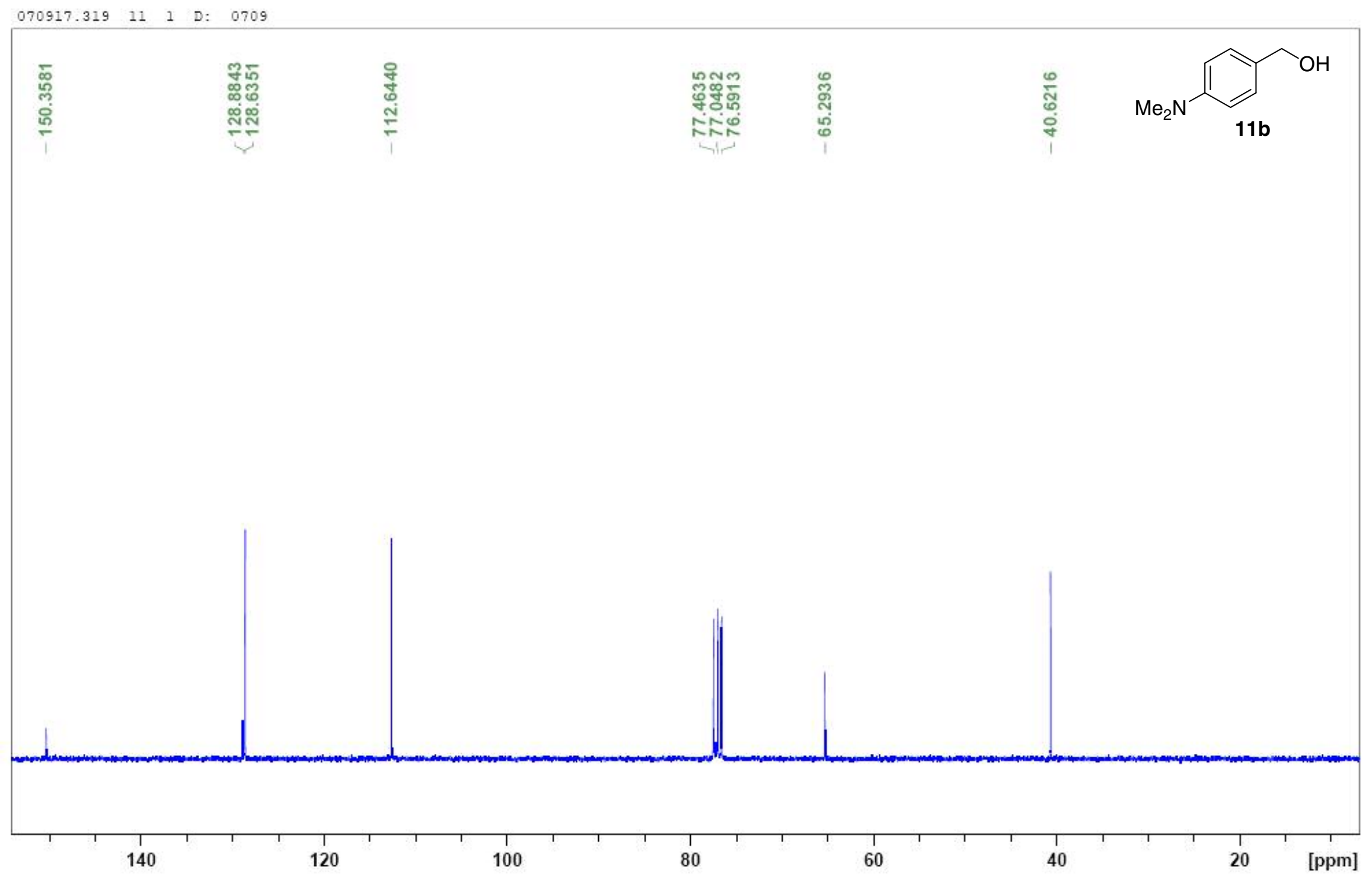


$070727.336 \quad 10 \quad 1$ D: 0707

Shaik, NS 3106

Au1H CDC13/opt/topspin 070736

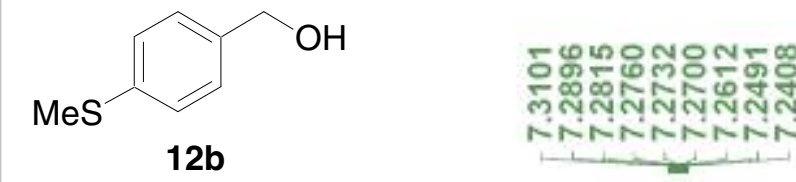

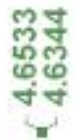

균

$12 b$

WHNNNA
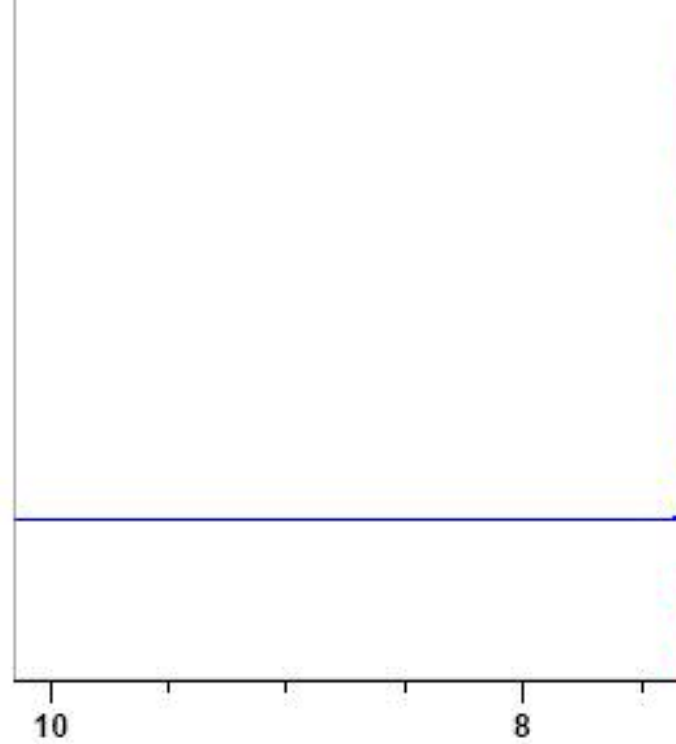

ปั
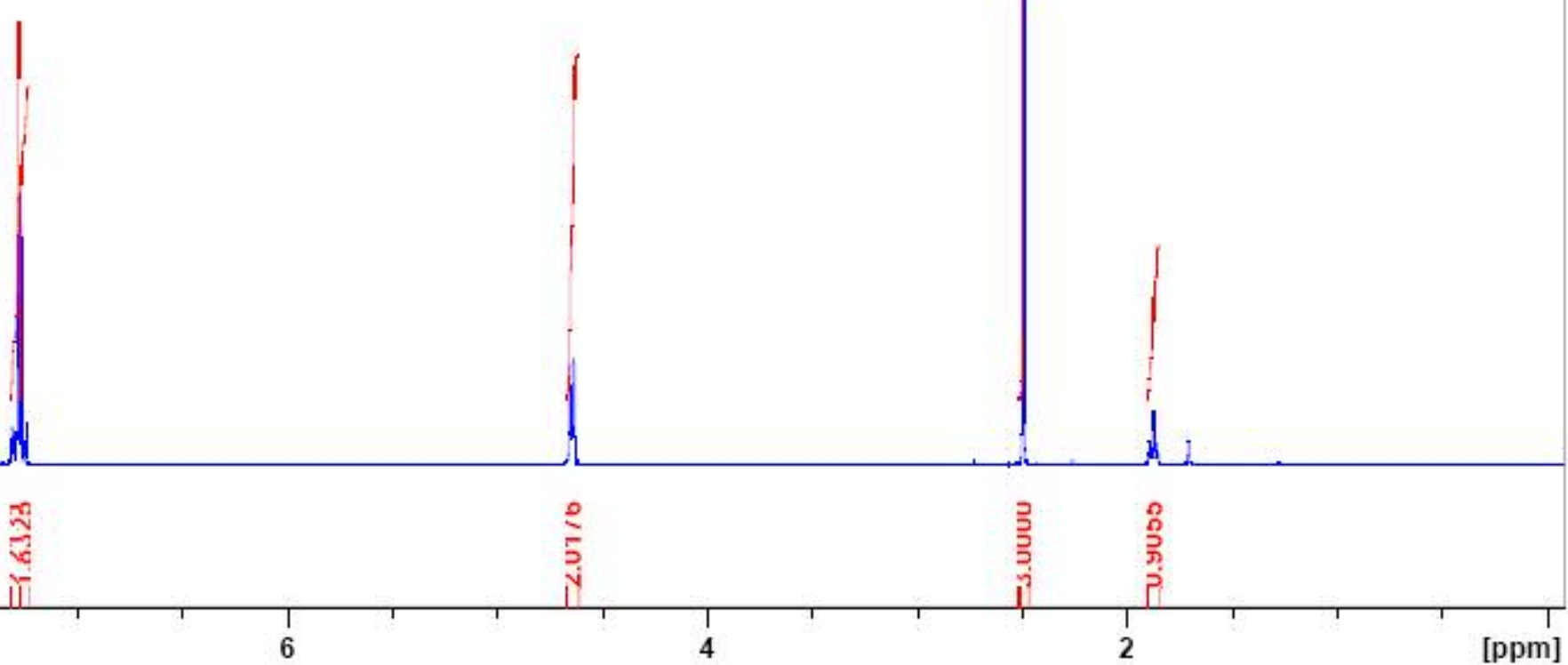
$070727.336 \quad 11 \quad 1 \quad$ D: 0707

Shaik, NS 3106
Aul3C CDC13/opt/topspin 070736
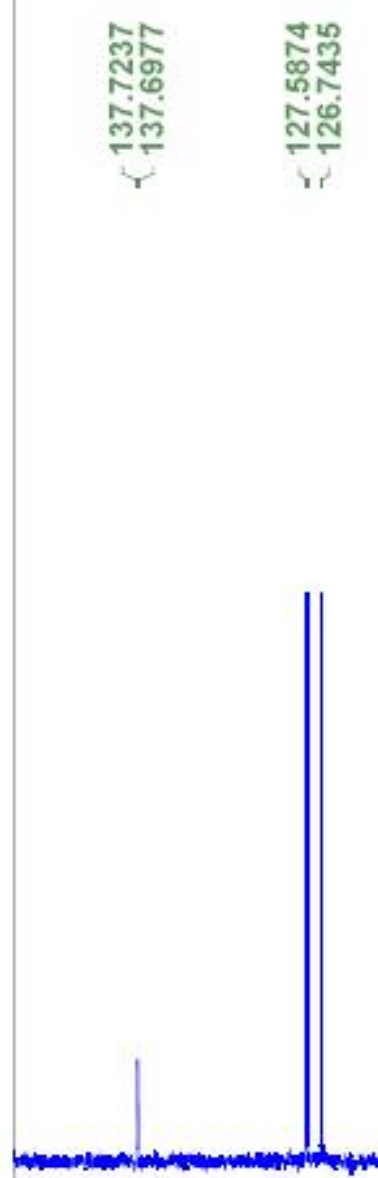


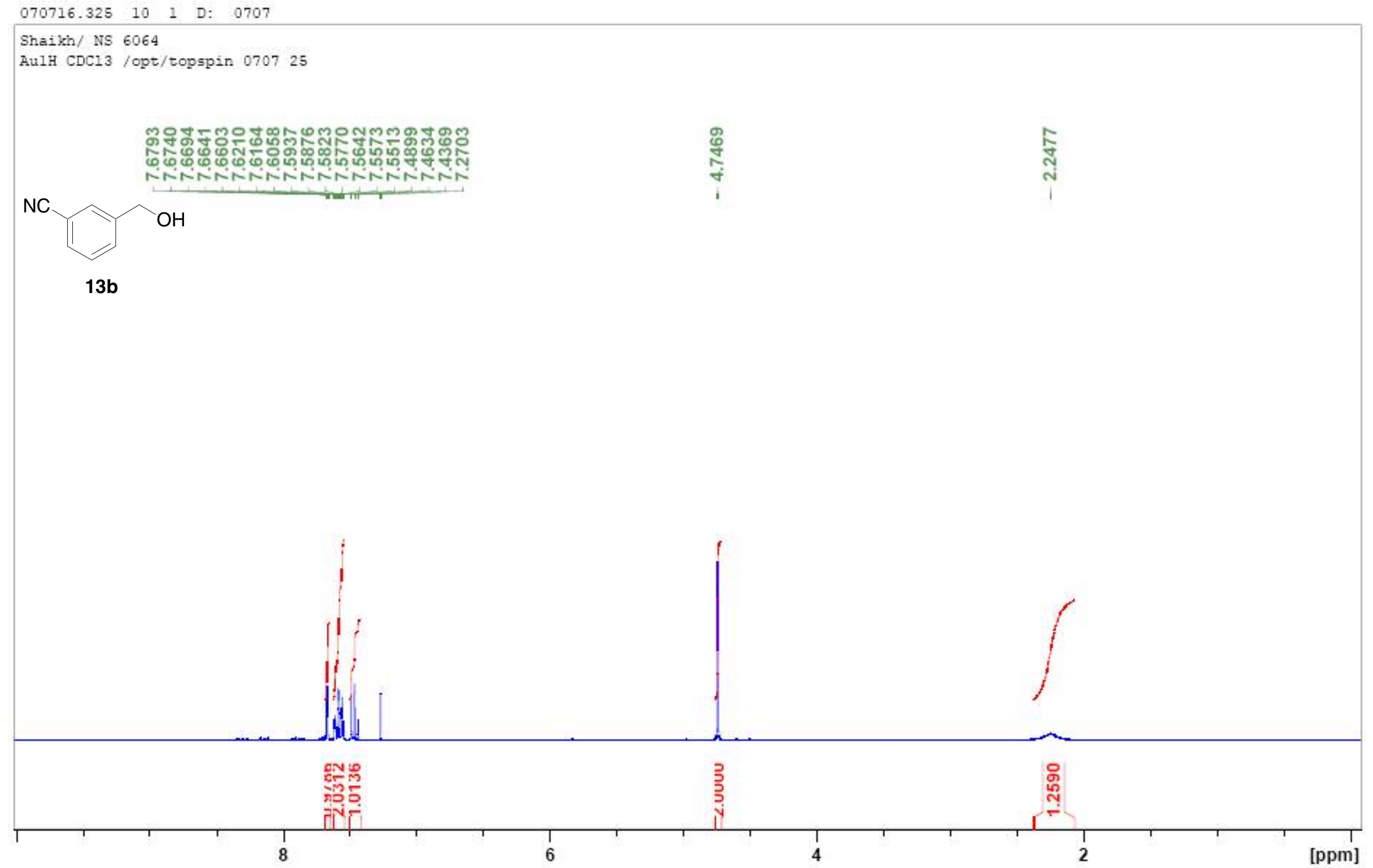


$070716.325 \quad 11 \quad 1 \quad$ D: 0707

Shaikh/NS 6064

Au13C CDC13 /opt/topspin 070725

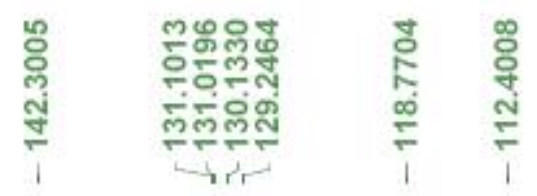

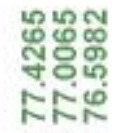

용

NC

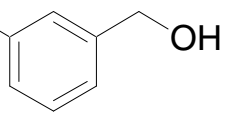

13b

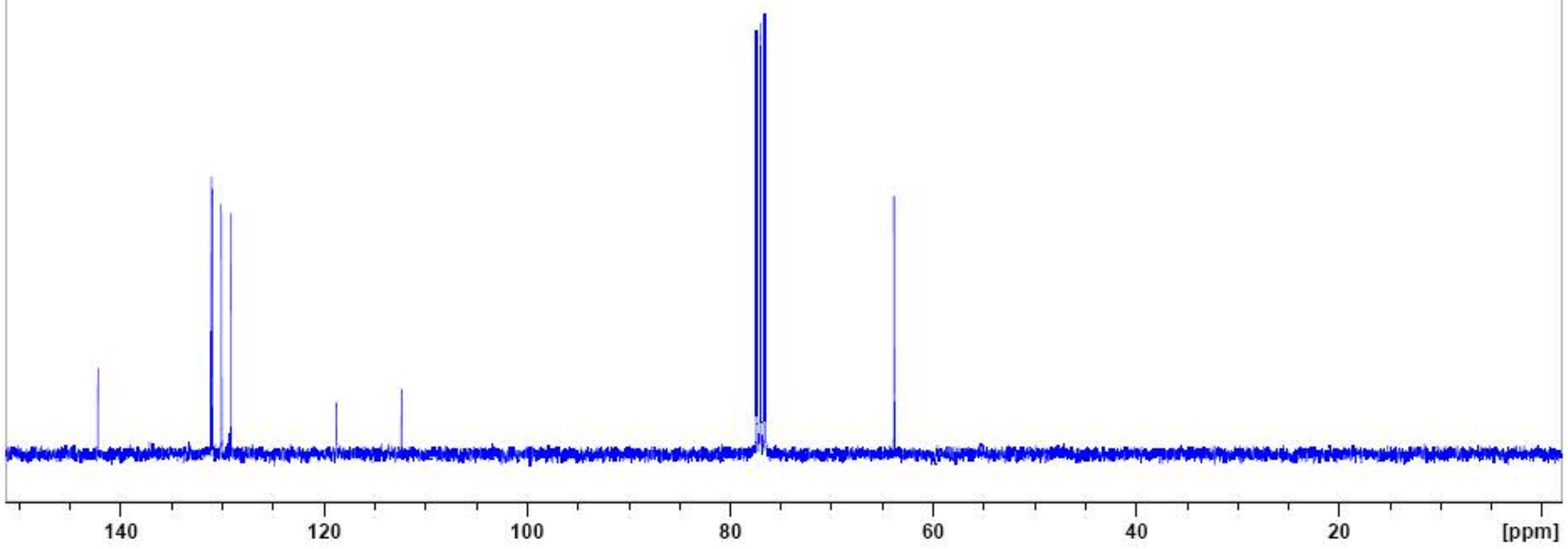


$070914.323 \quad 10 \quad 1 \quad$ D: 0709

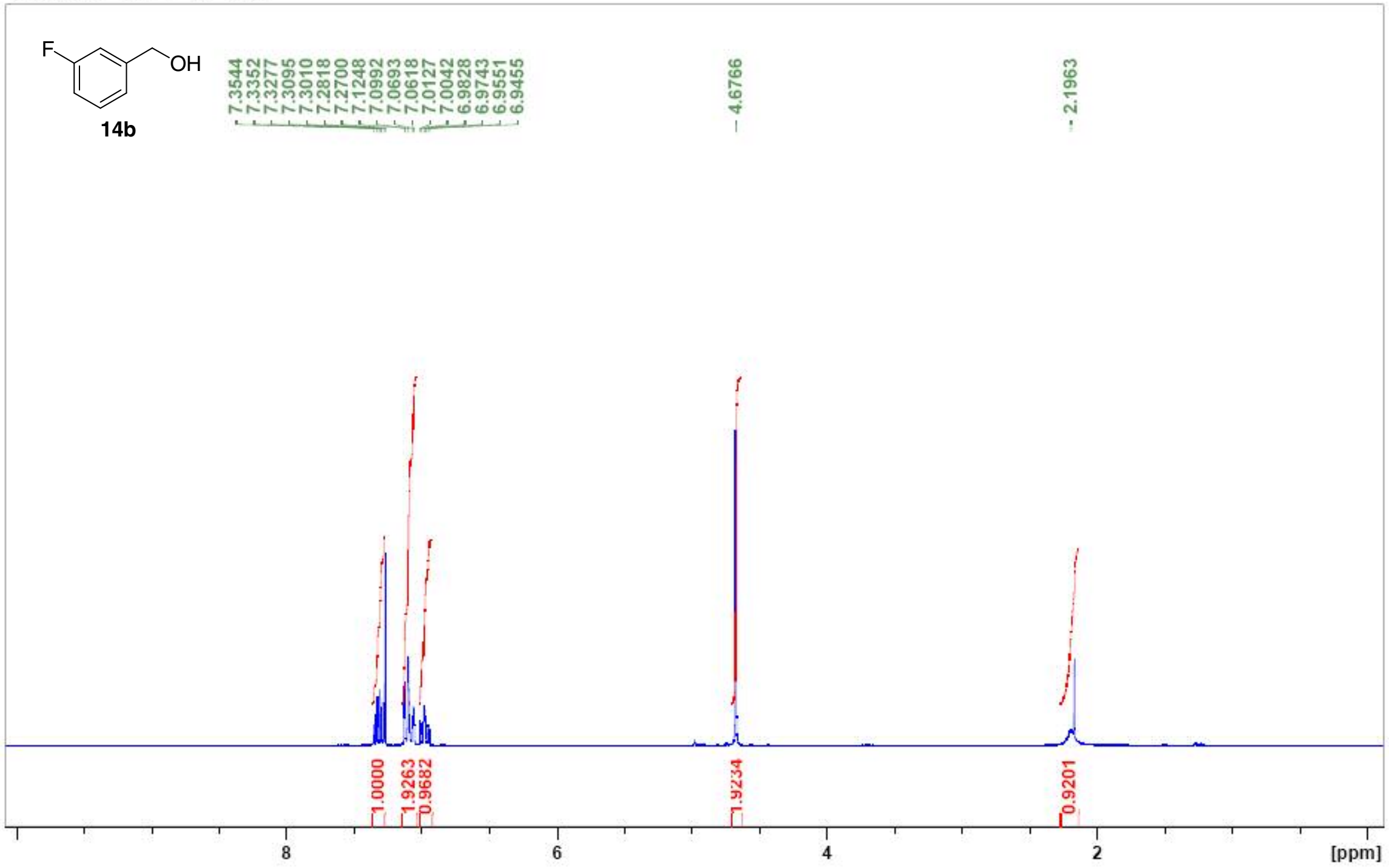


Supporting information-2

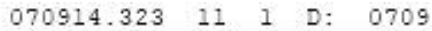

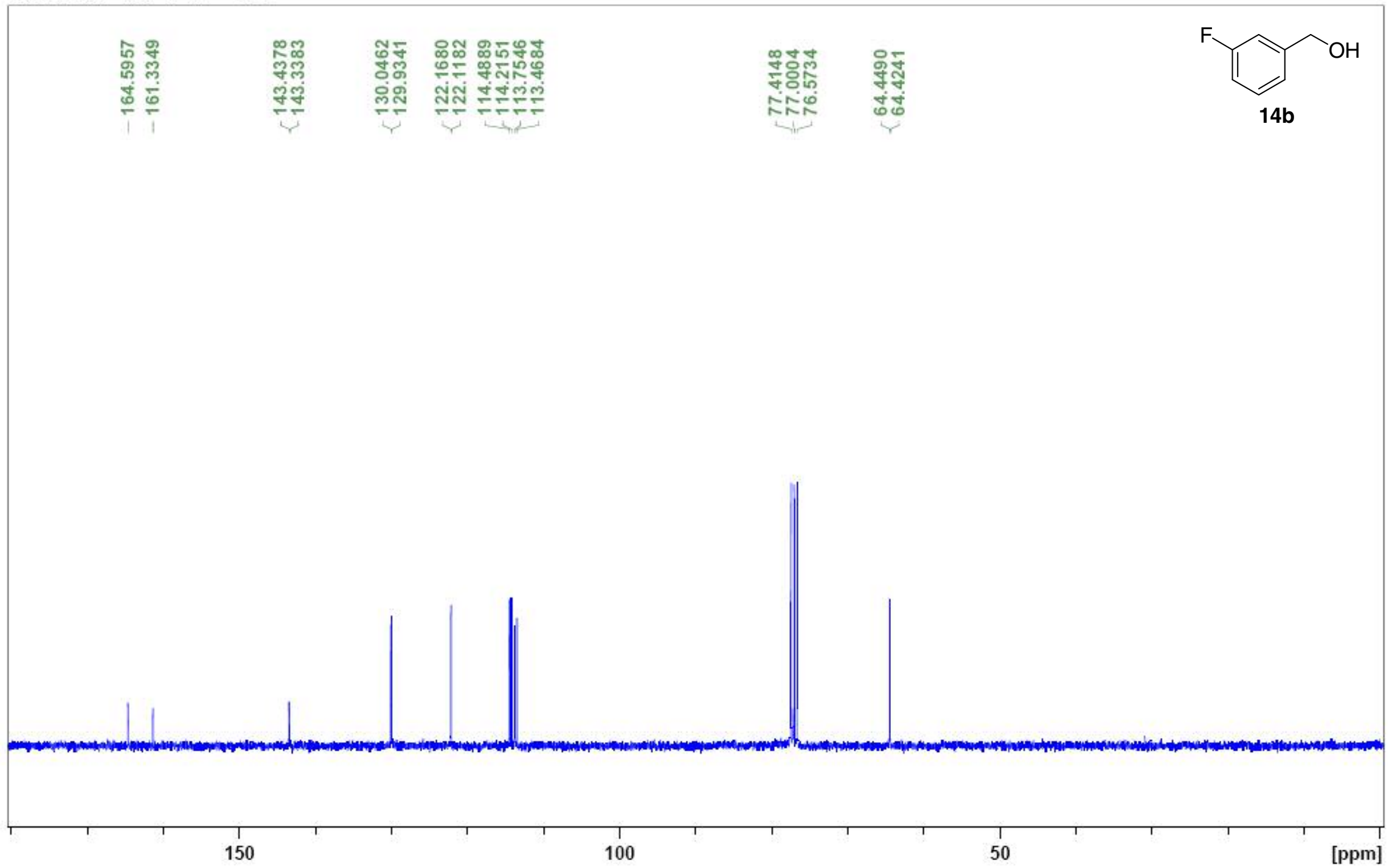


$070718.333 \quad 10 \quad 1 \quad$ D: 0707

Shaikh, NS 6068

AulH CDC13 /opt/topspin 070733

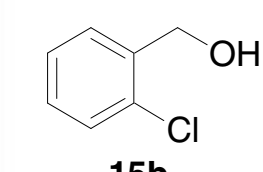

15b

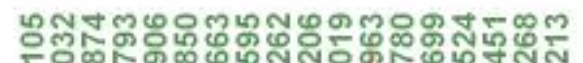

non

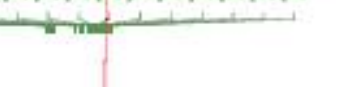

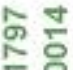

กั่

i 


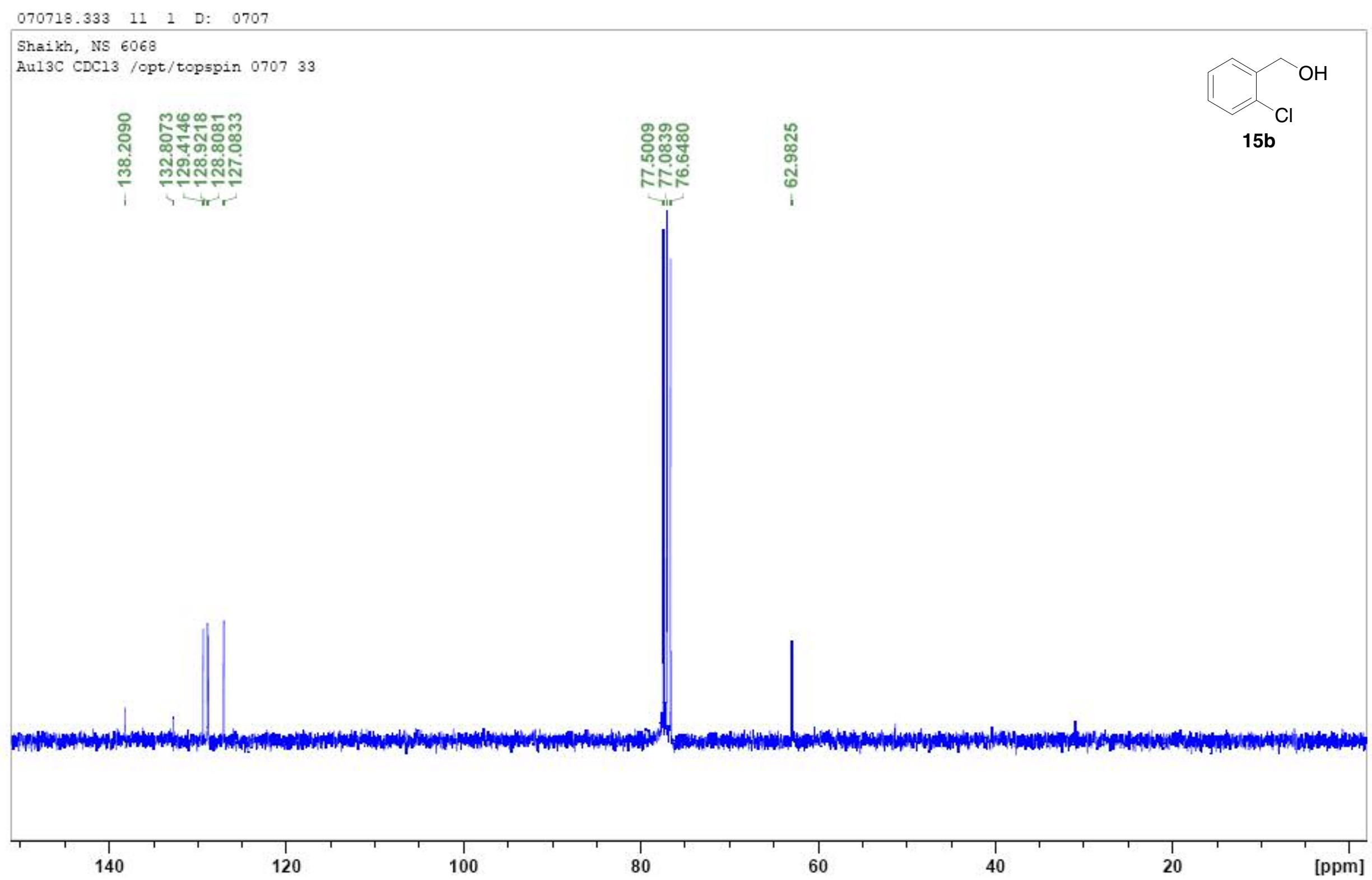




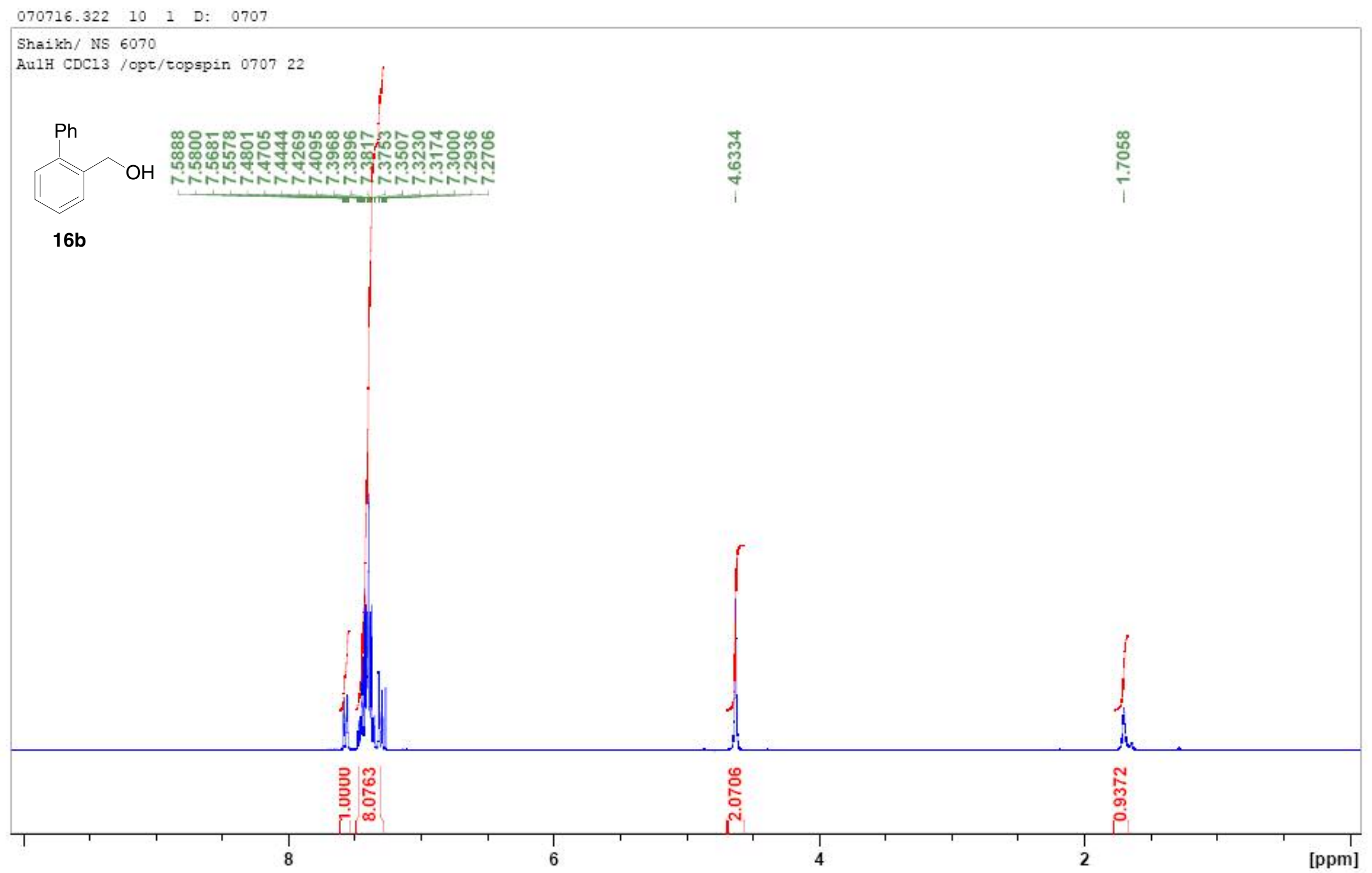




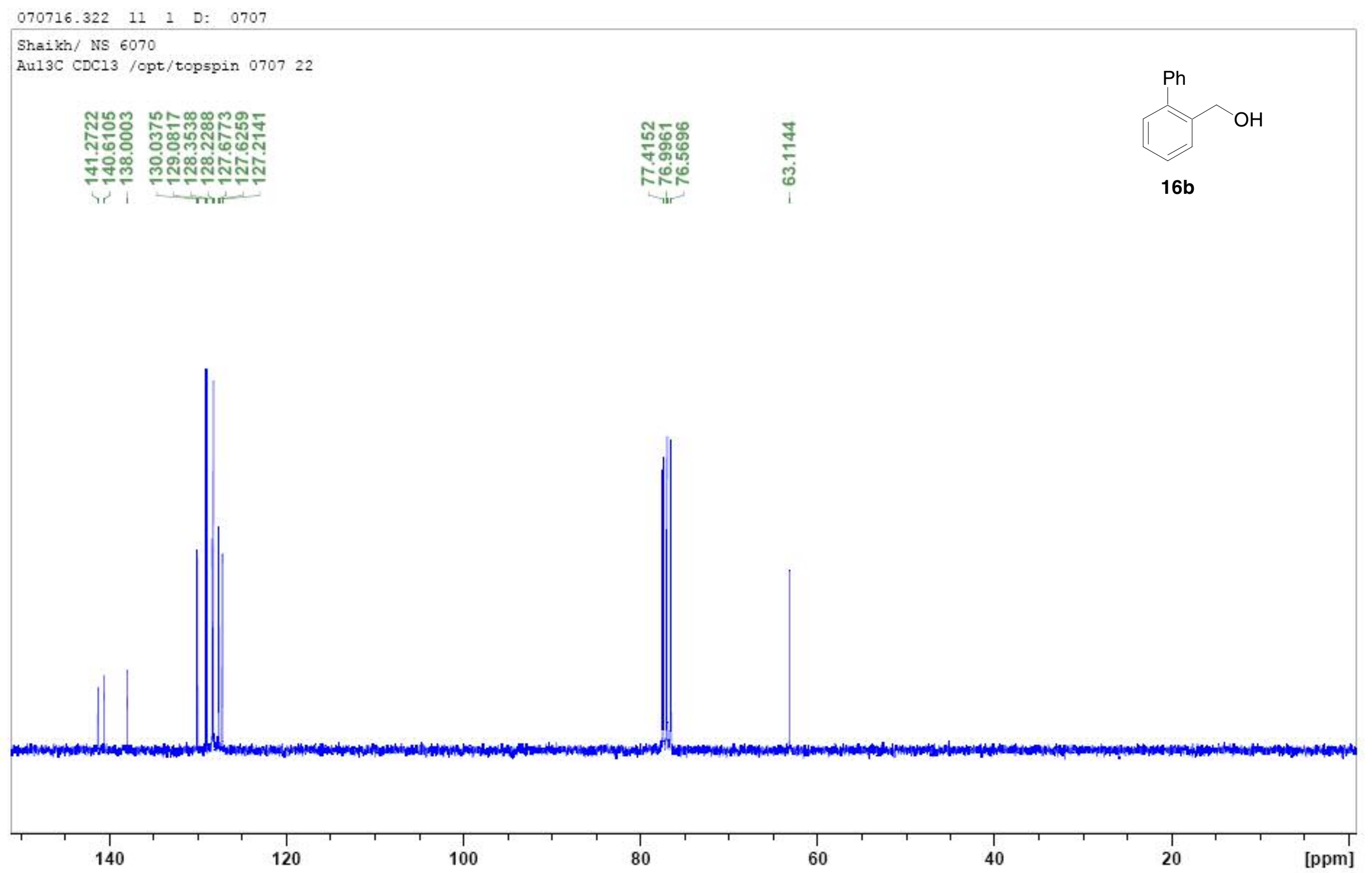




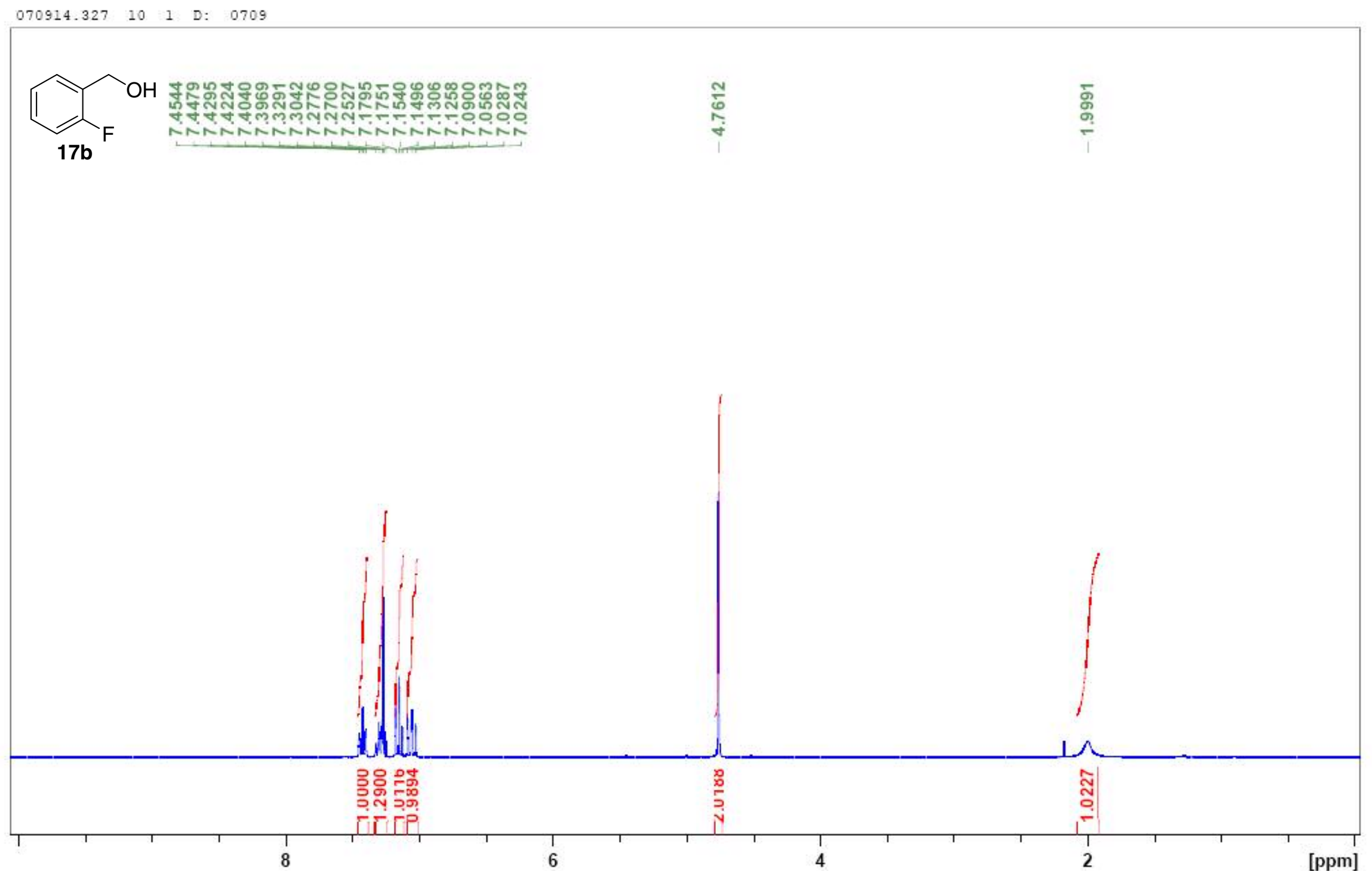




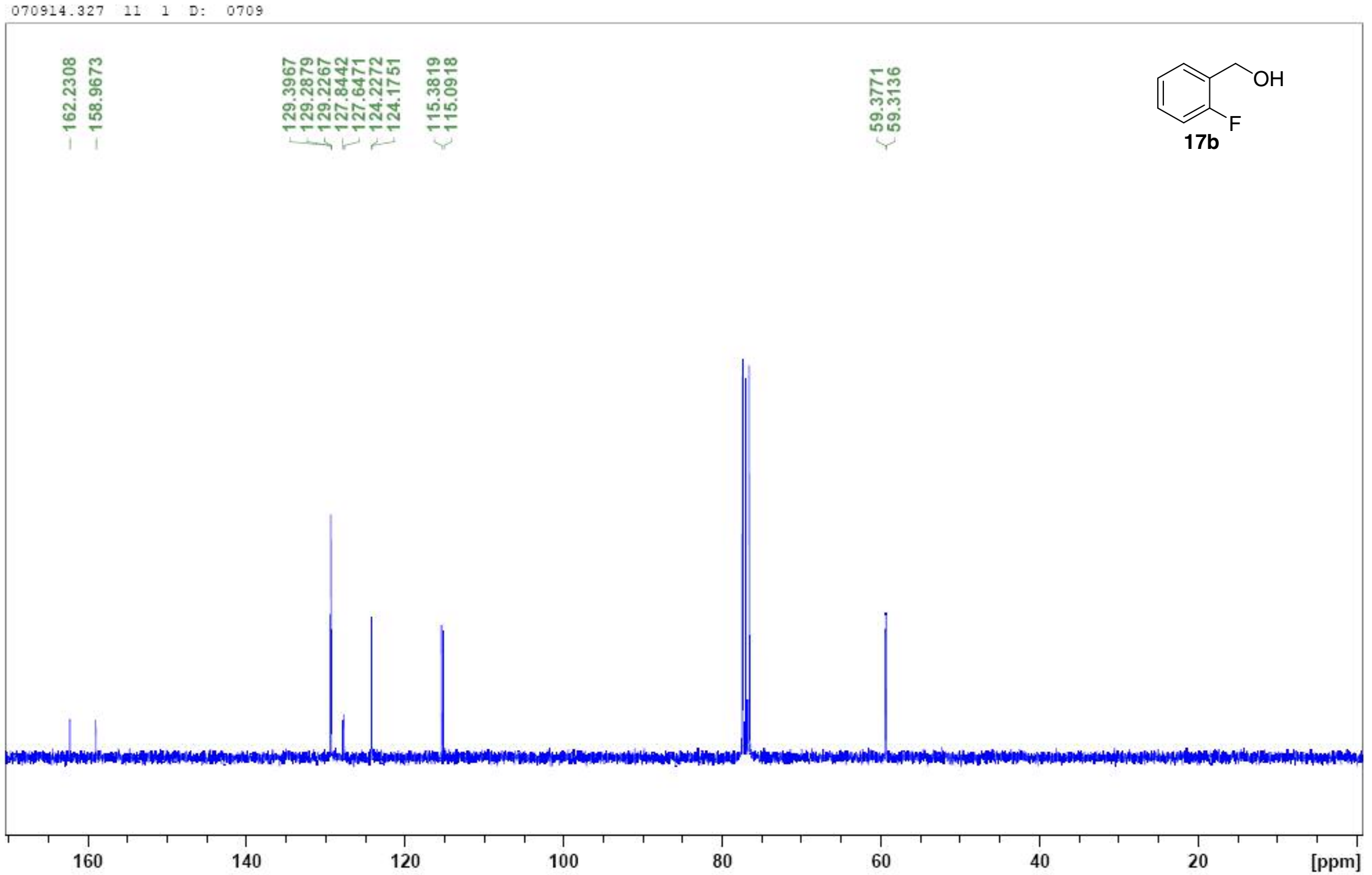




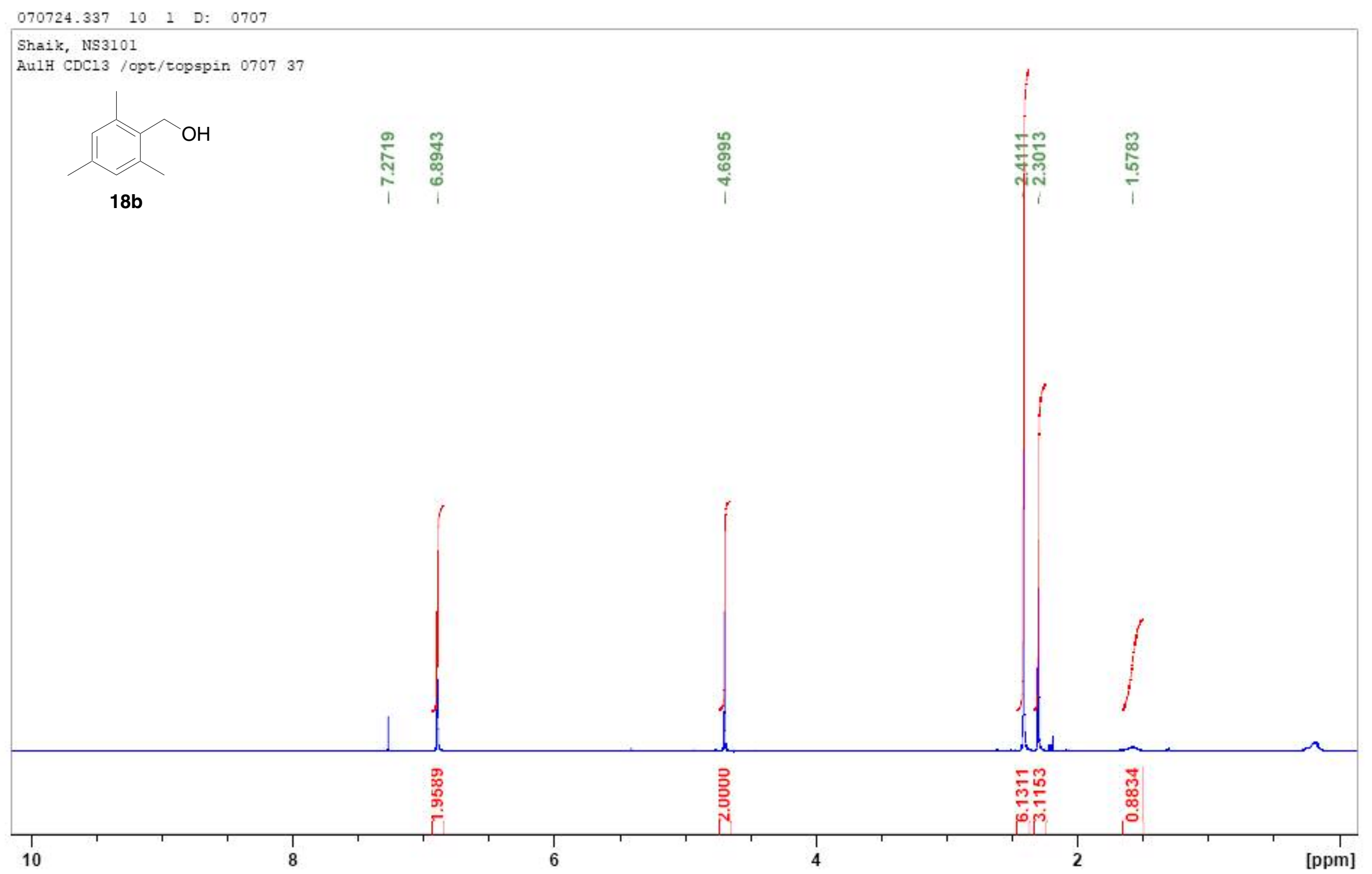




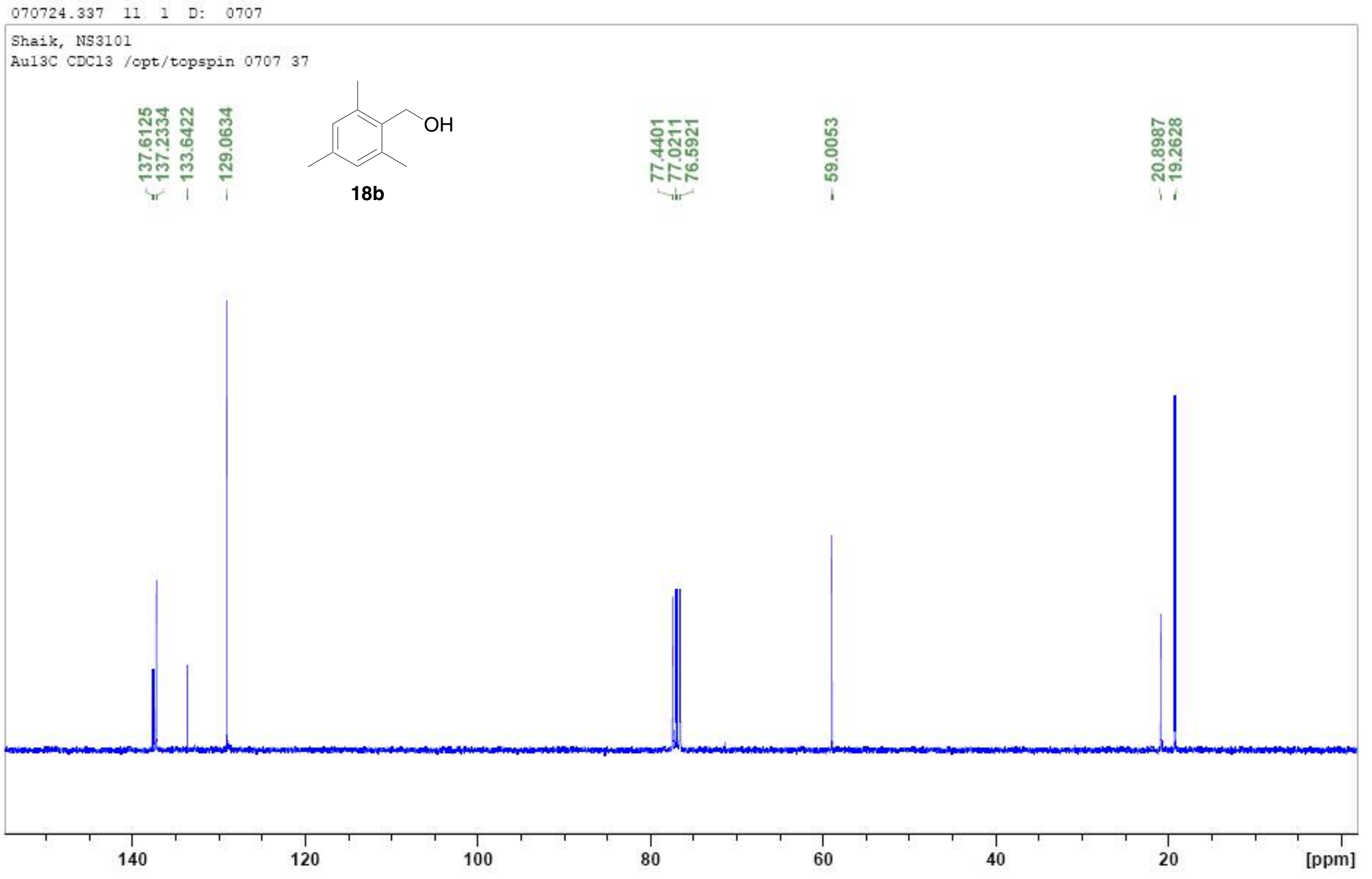




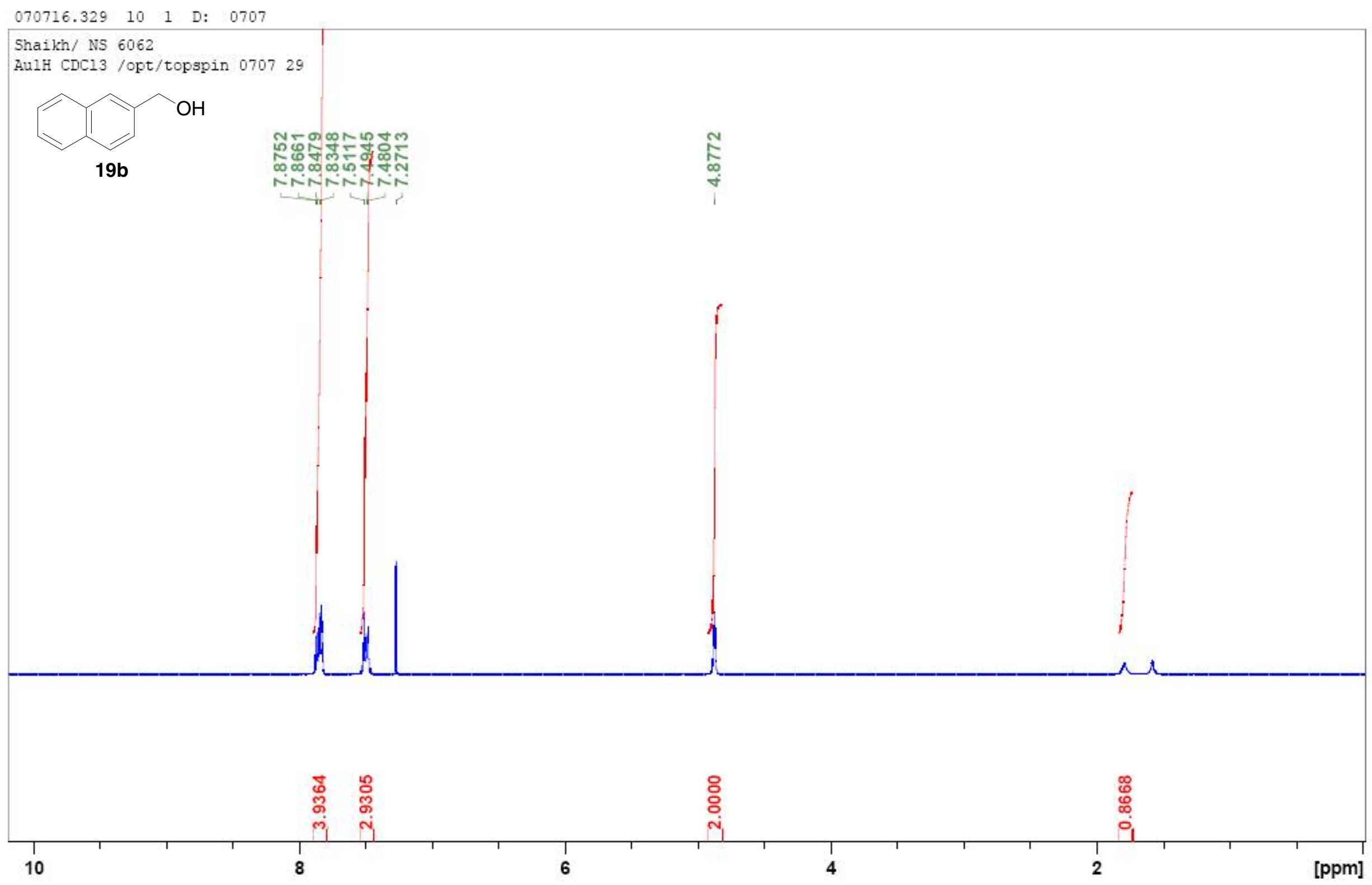




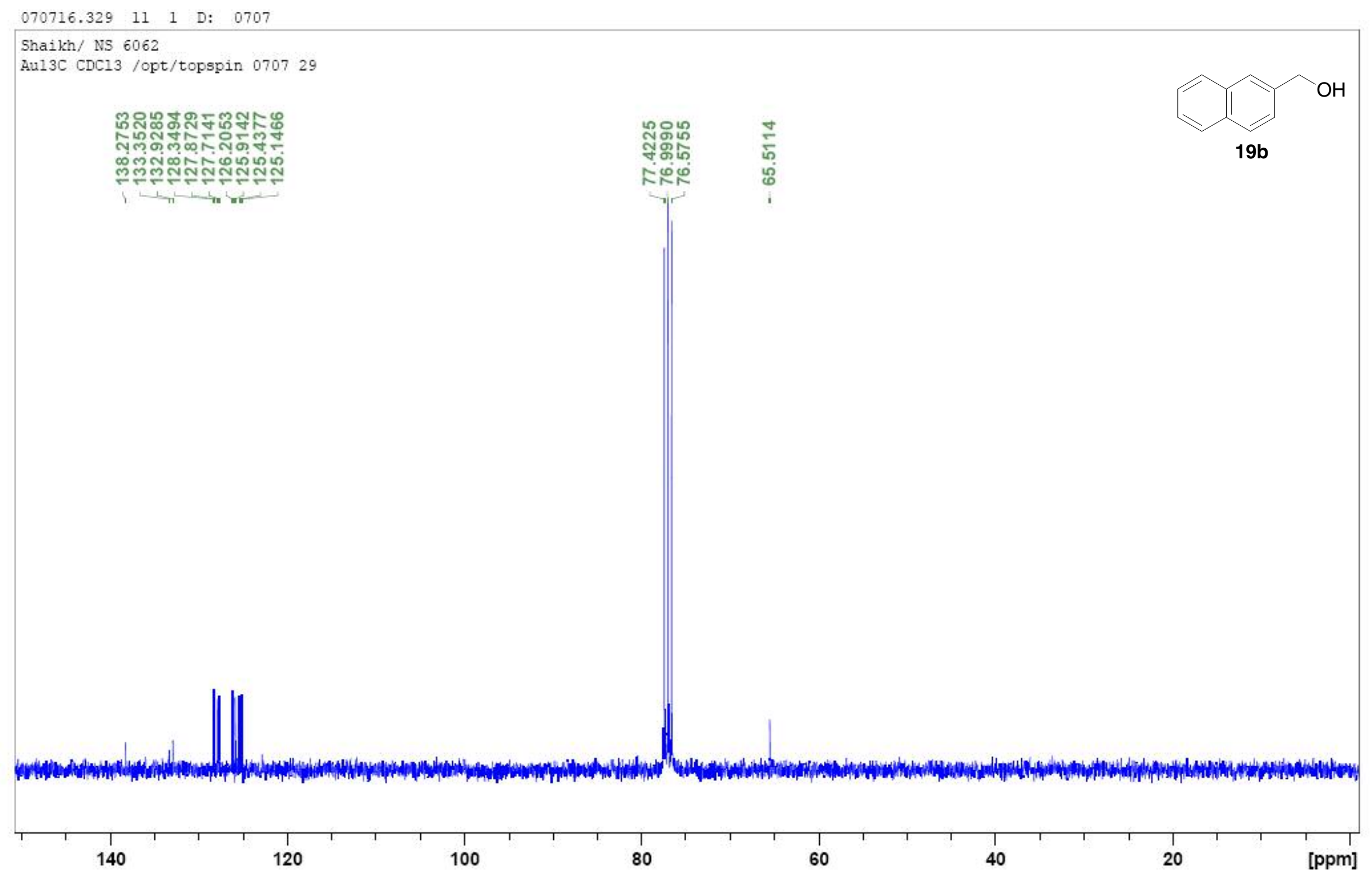


$070716.328 \quad 10 \quad 1 \quad$ D: 0707

Shaikh/ NS 6063

AulH CDC13/opt/topspin 070728

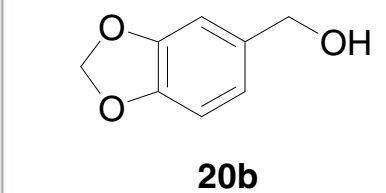

20b

mก

సิ心

-

Гूल

min

रTल

둔

寸

is
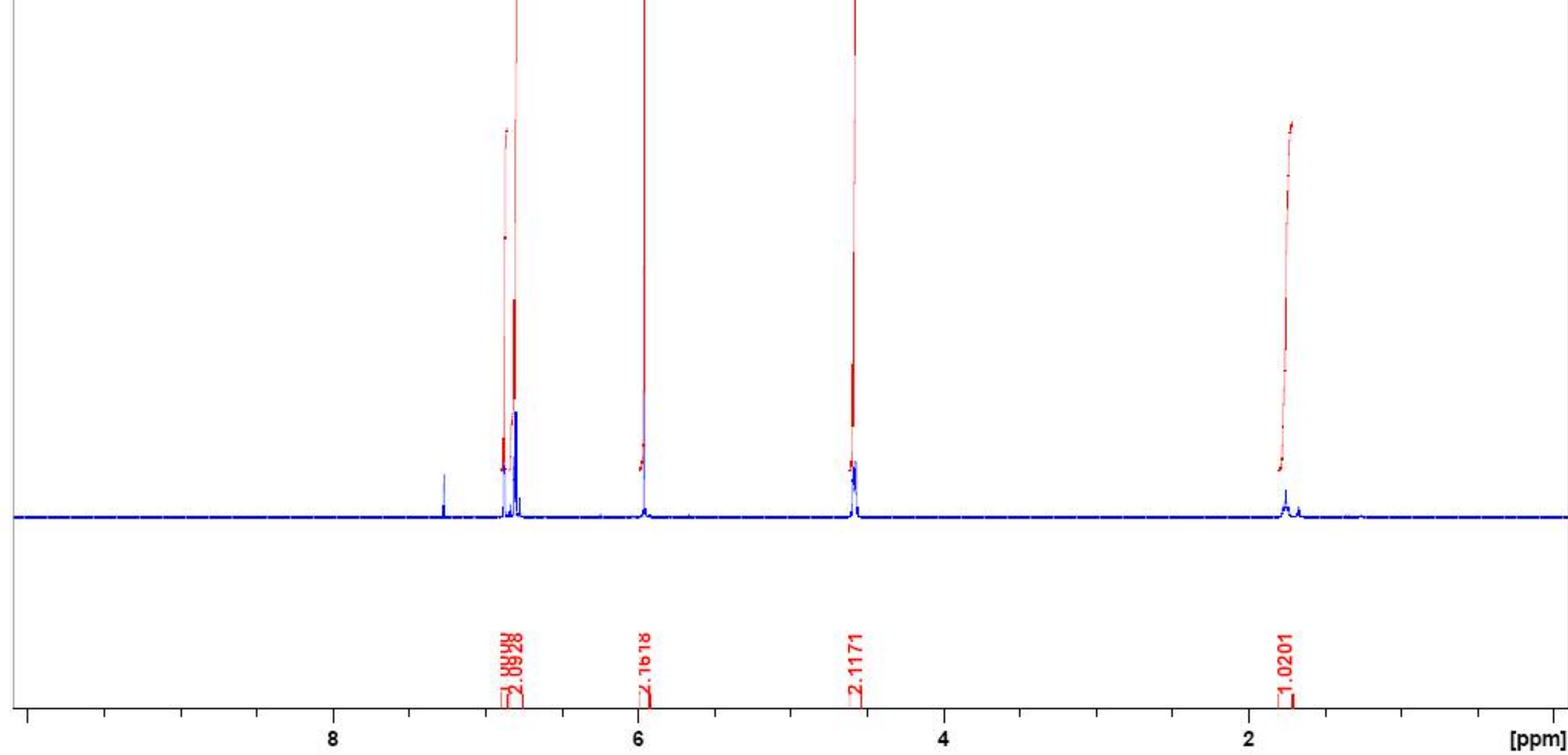


\section{$070716.328 \quad 11 \quad 1$ D: 0707}

Shaikh/ NS 6063

Au13C CDC13/opt/topspin 070728

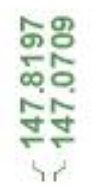

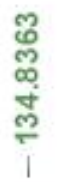

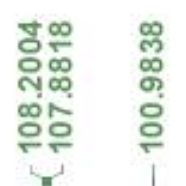

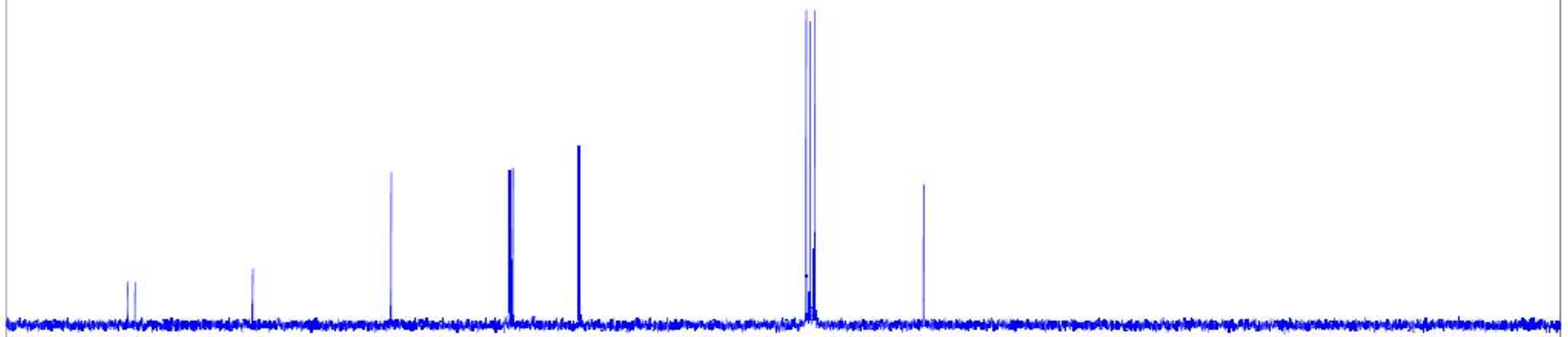

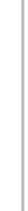

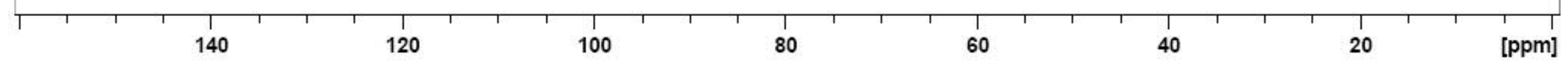




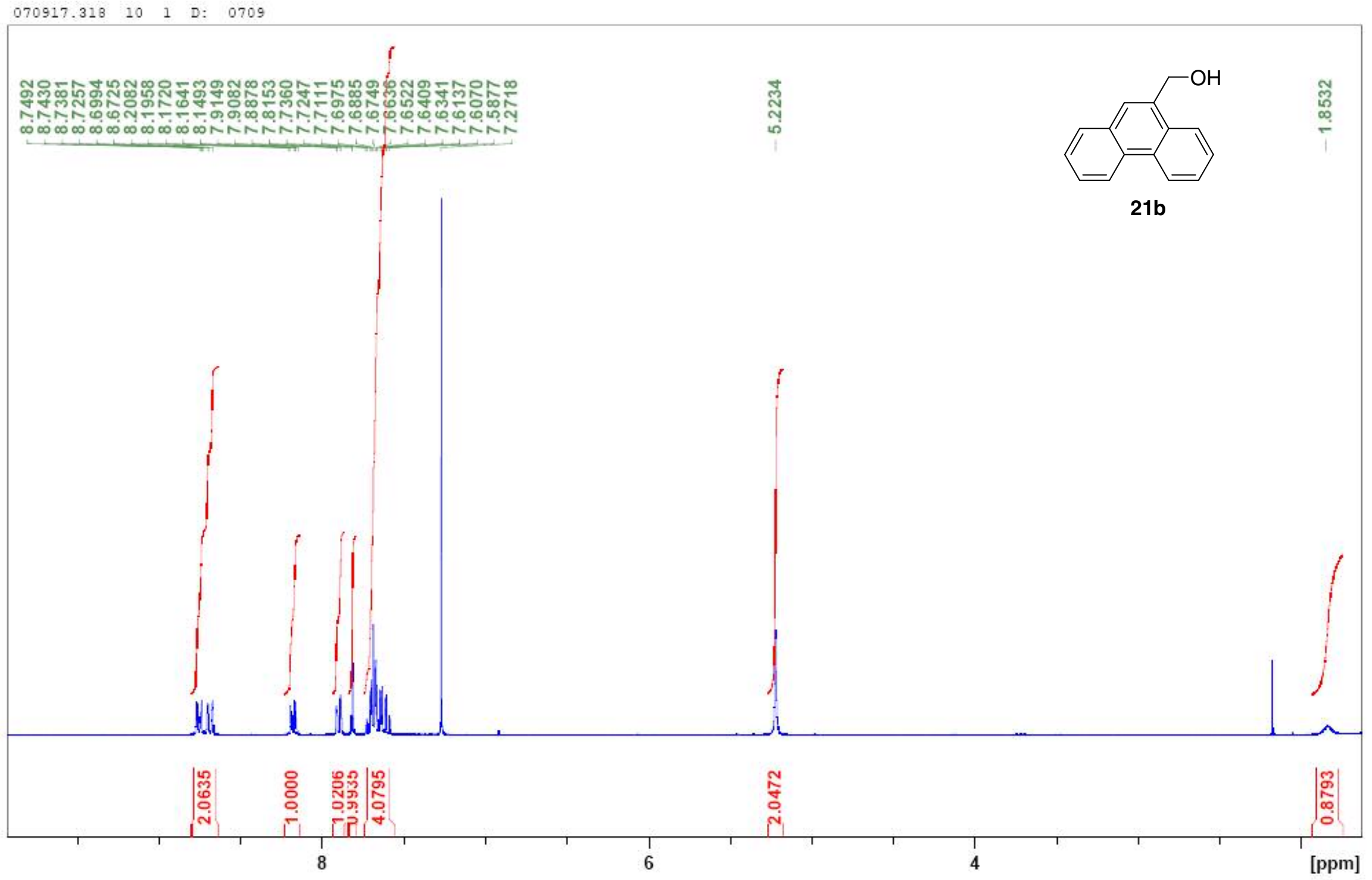




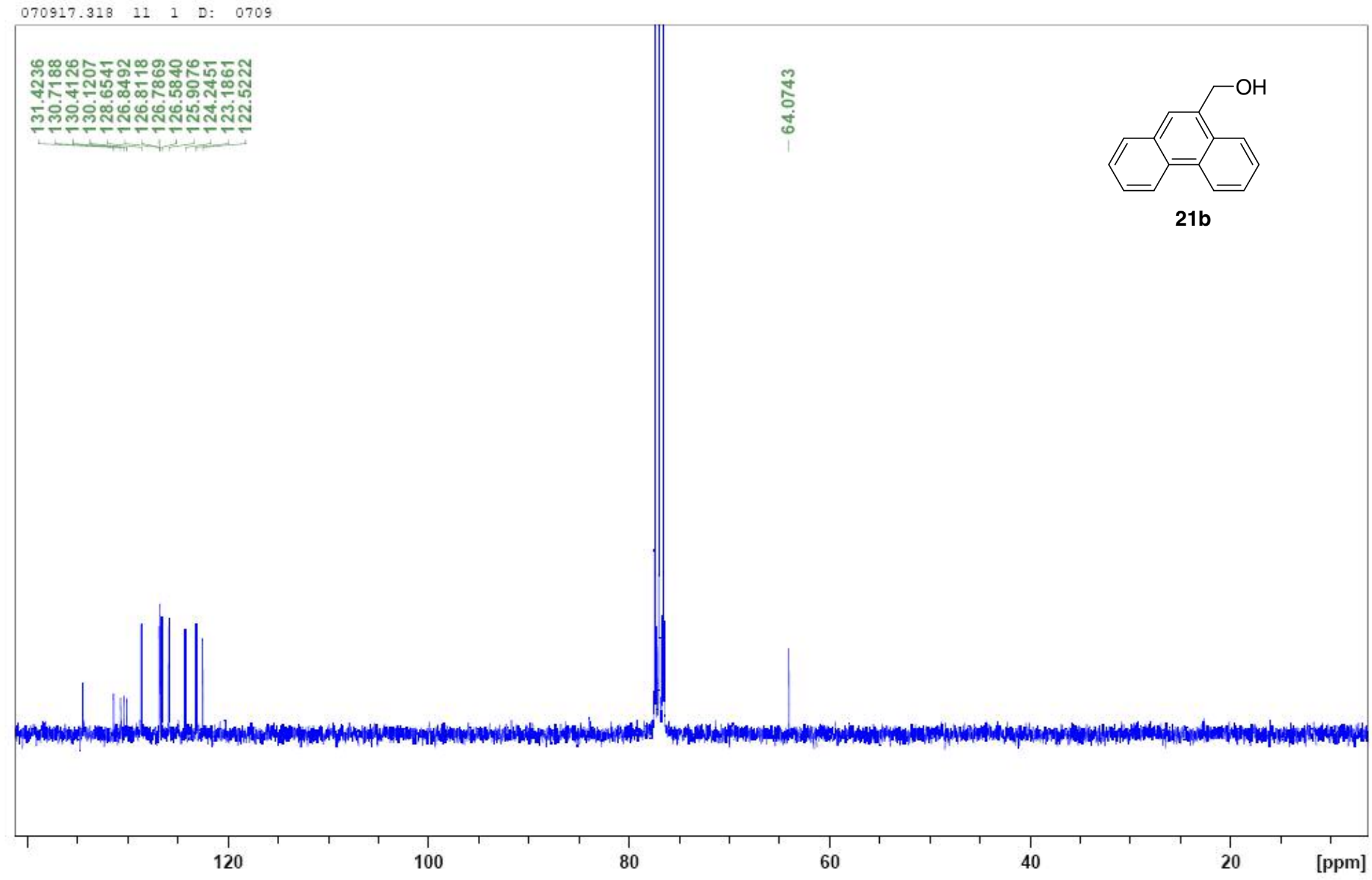




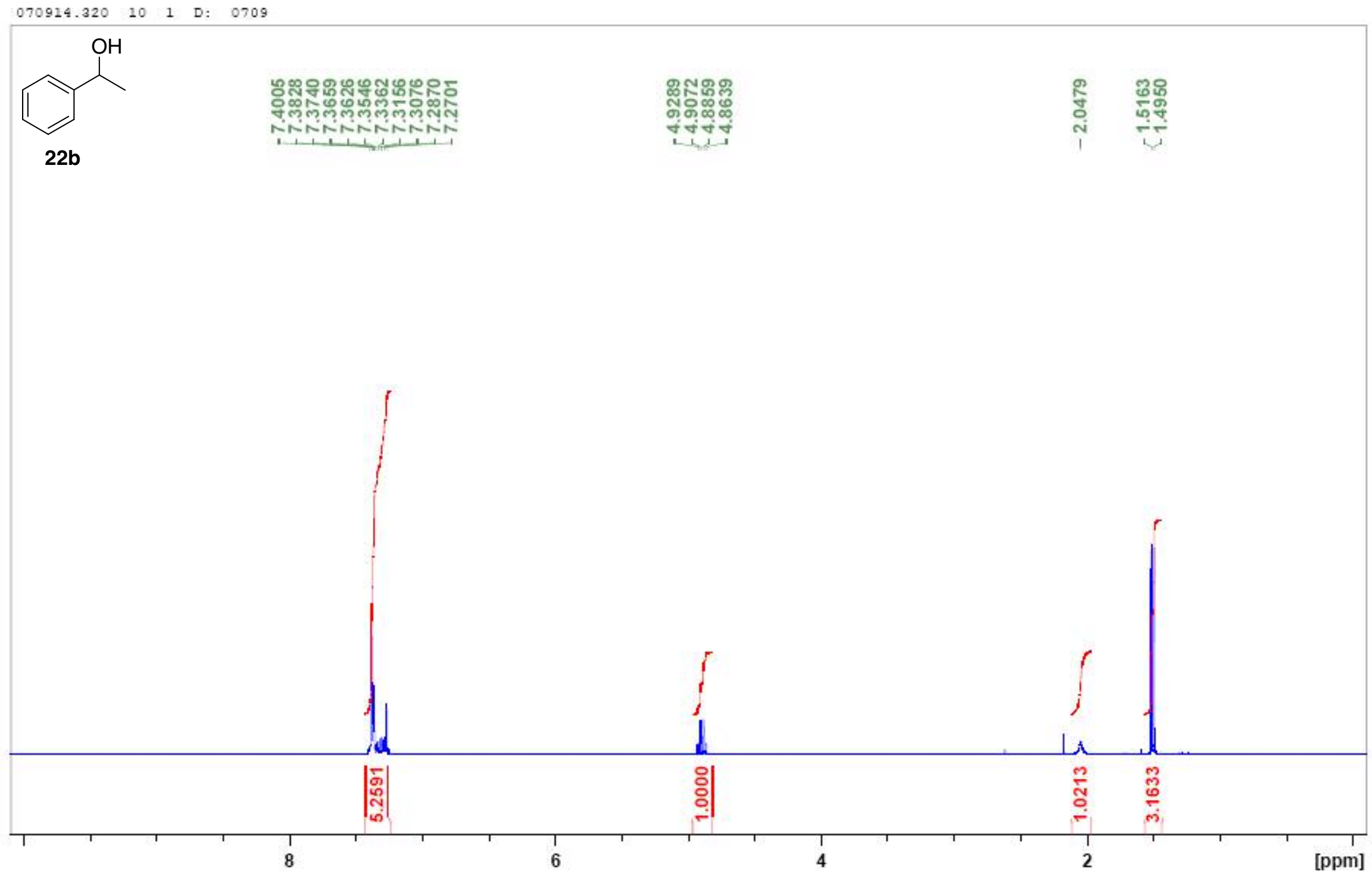


Supporting information-2

070914.32011 1 D: 0709

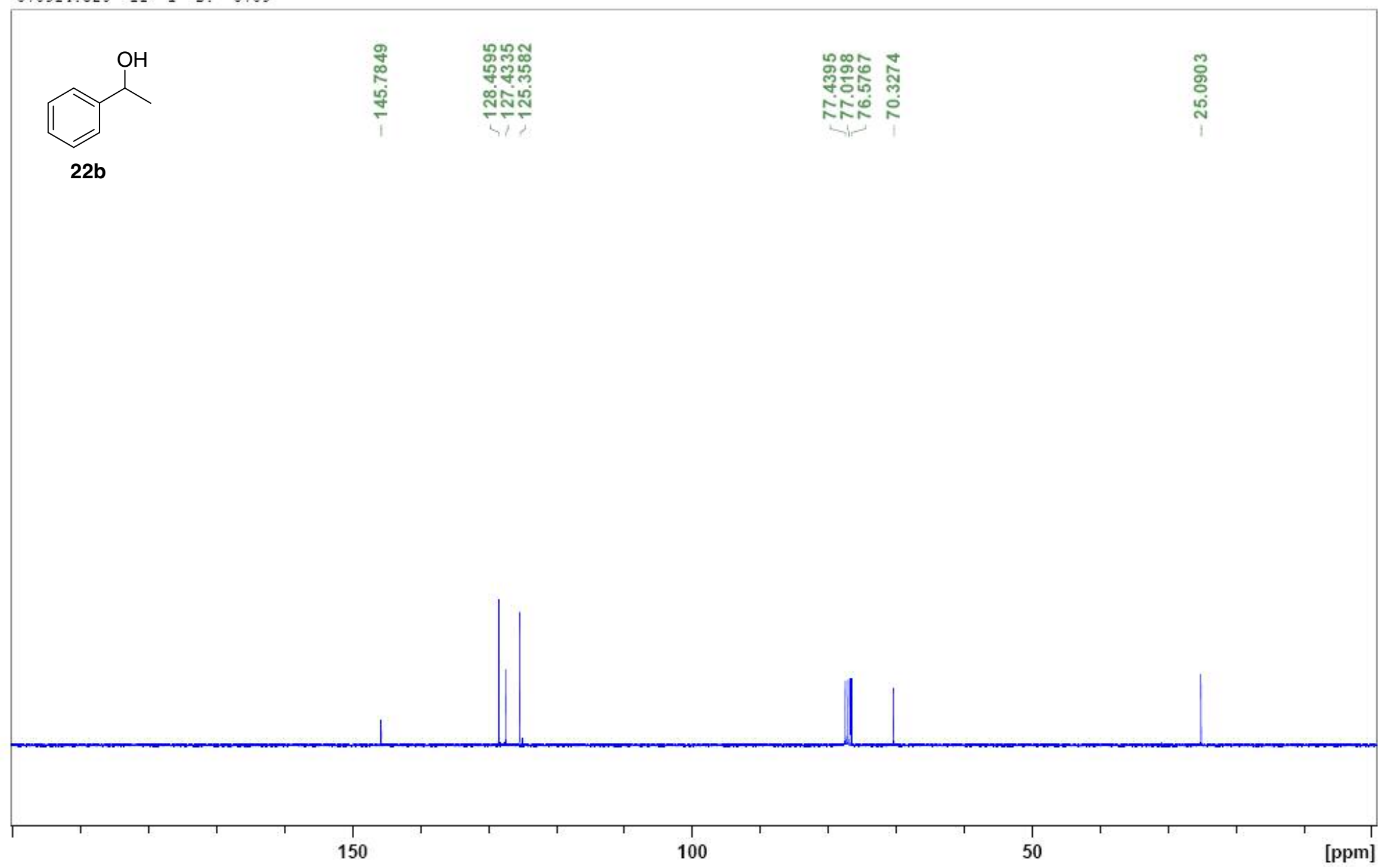


Supporting information-2

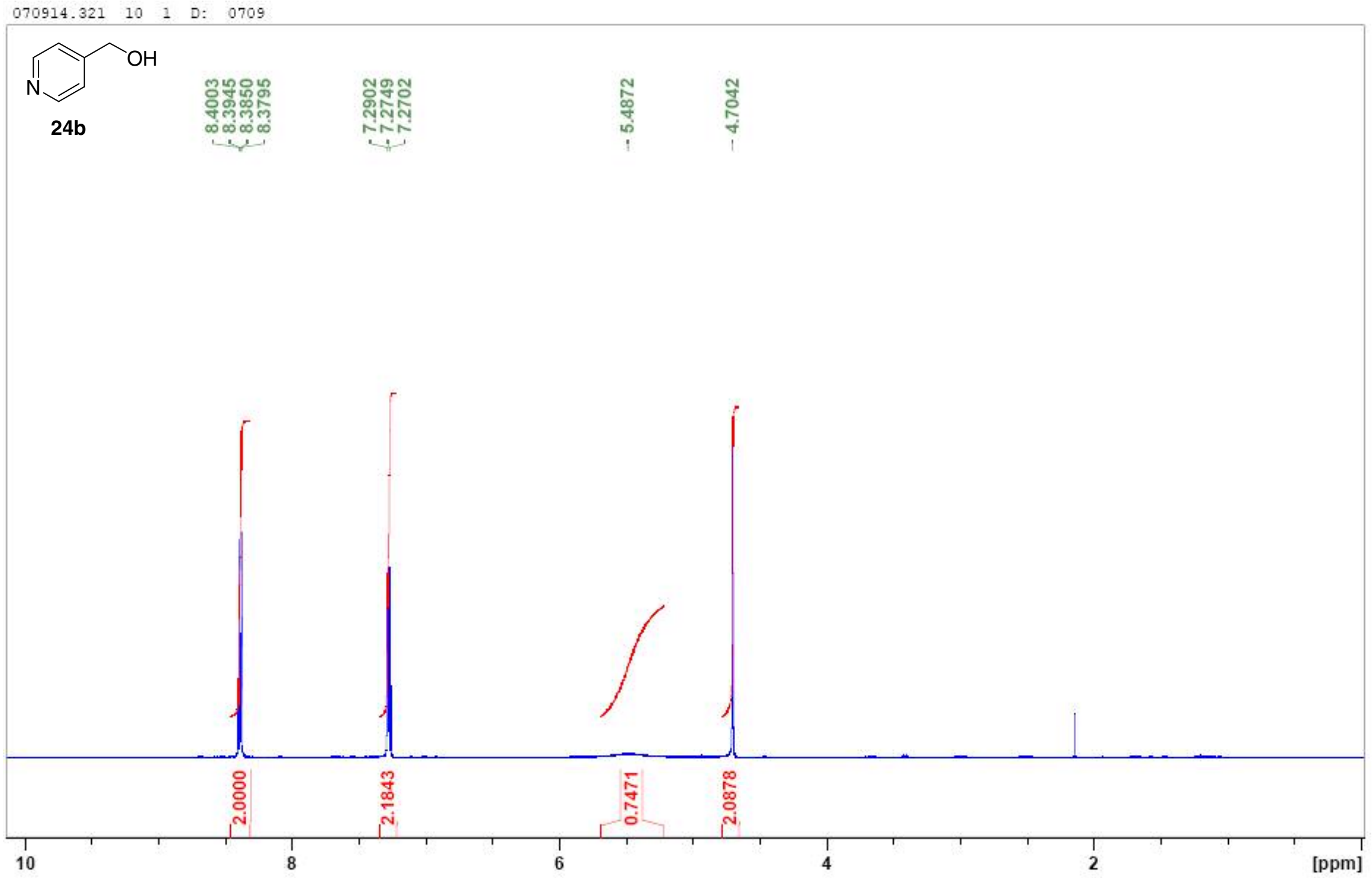


Supporting information-2

$070914.321 \quad 11 \quad 1 \quad$ D: 0709

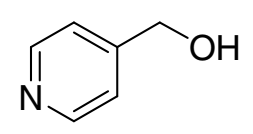

$24 b$
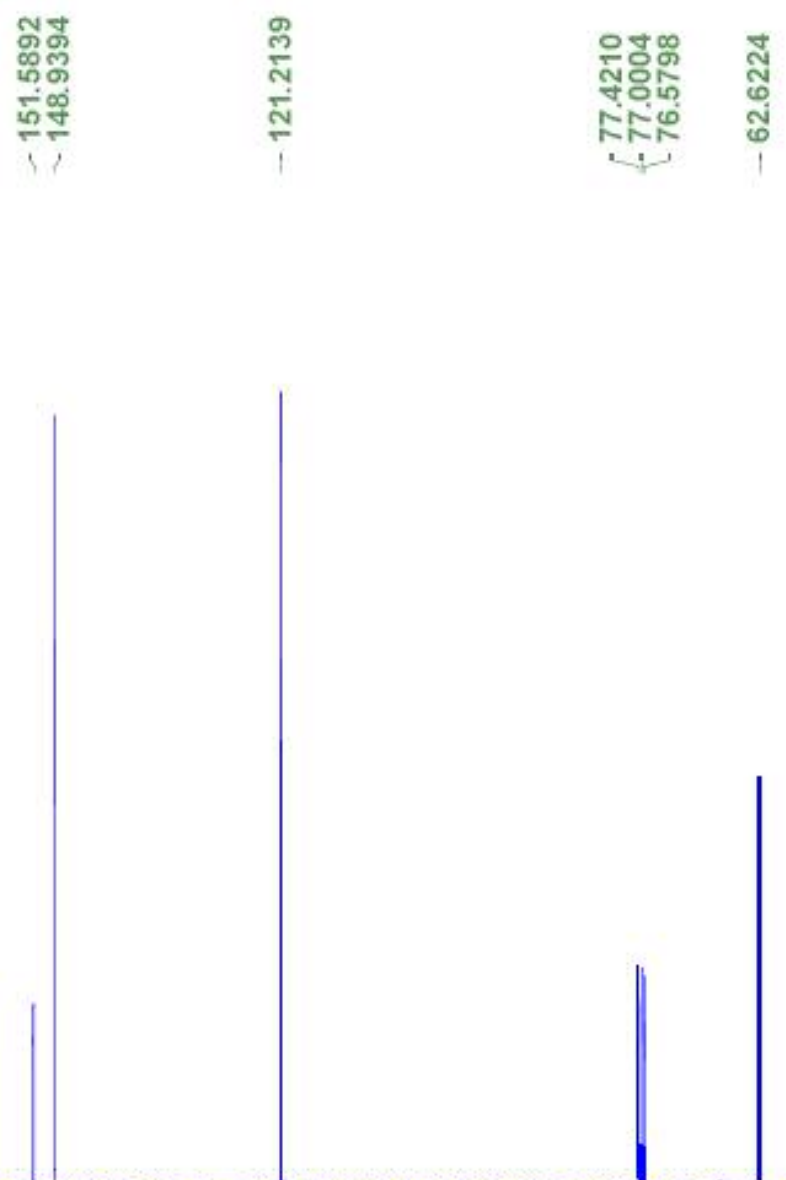


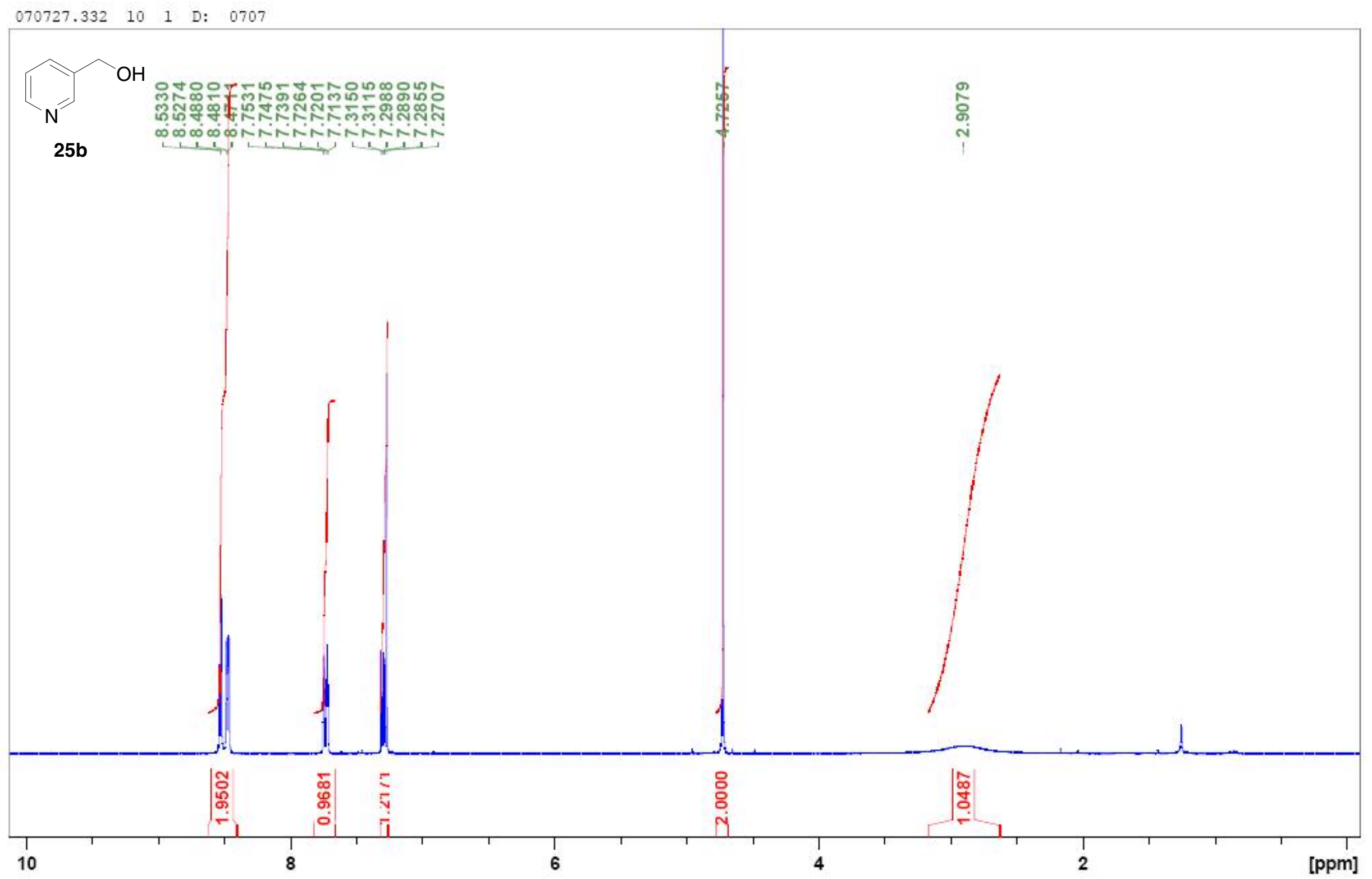


$070727.332 \quad 11 \quad 1 \quad$ D: 0707

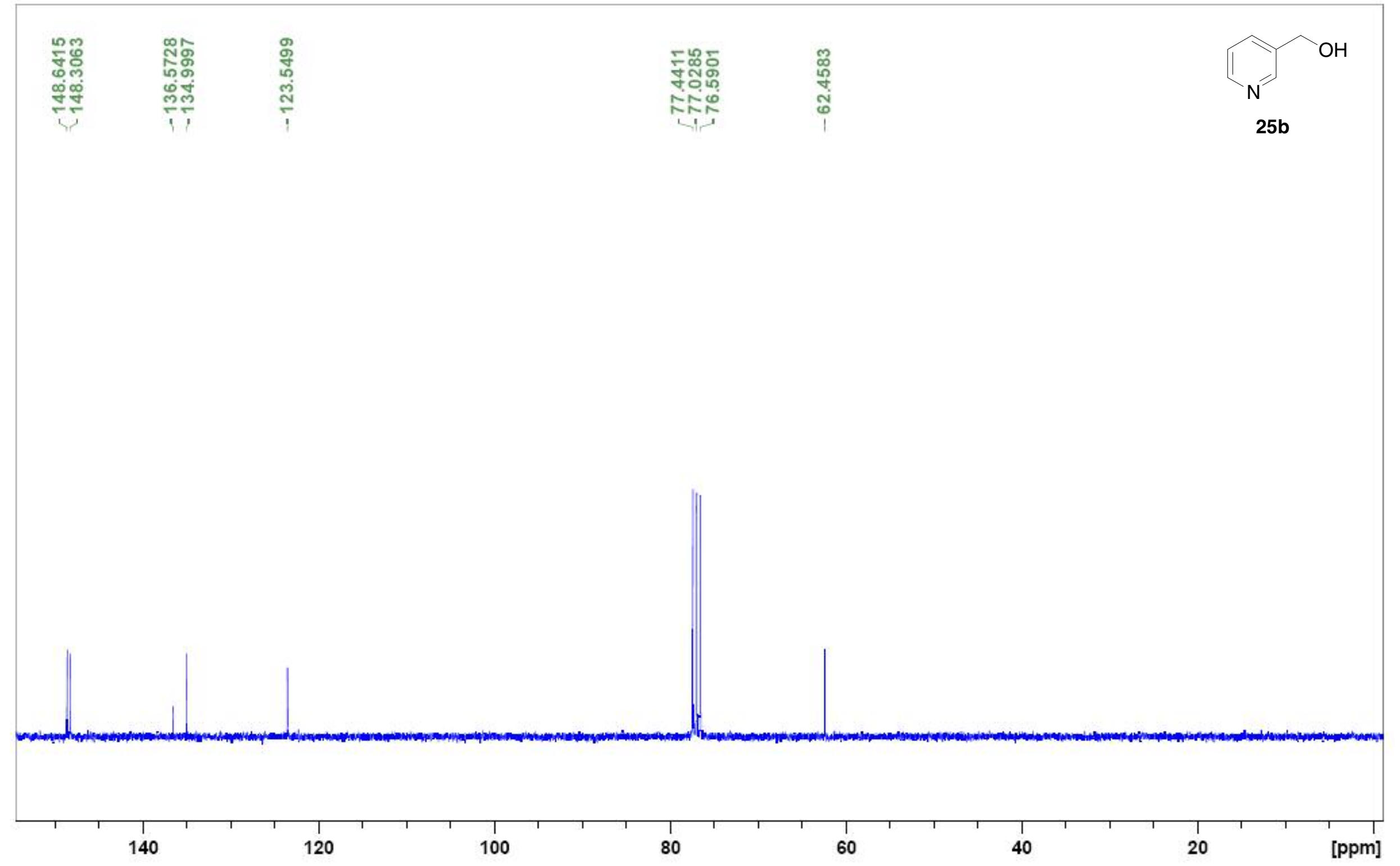




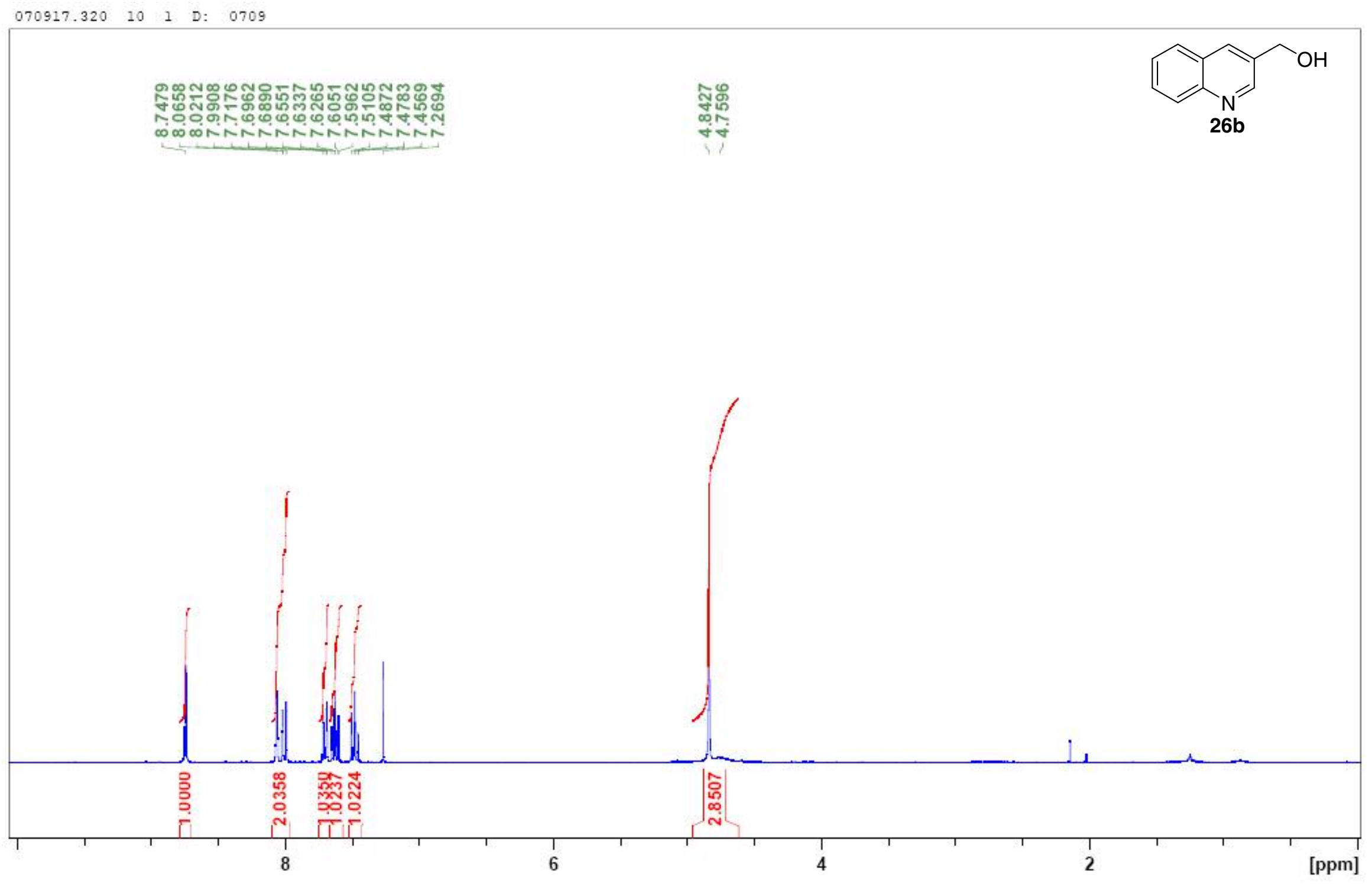


$070917.320 \quad 11 \quad 1 \quad$ D: 0709

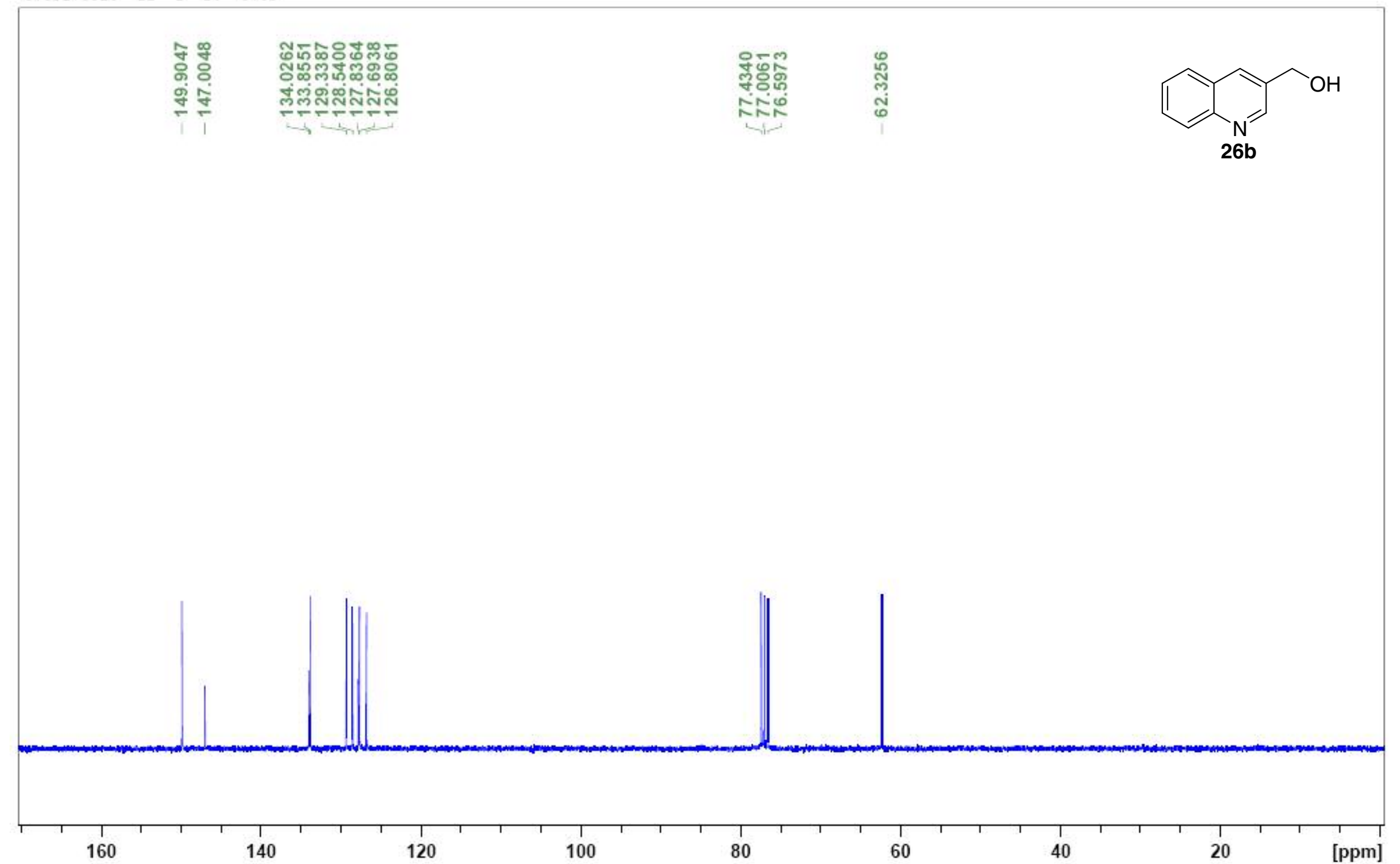




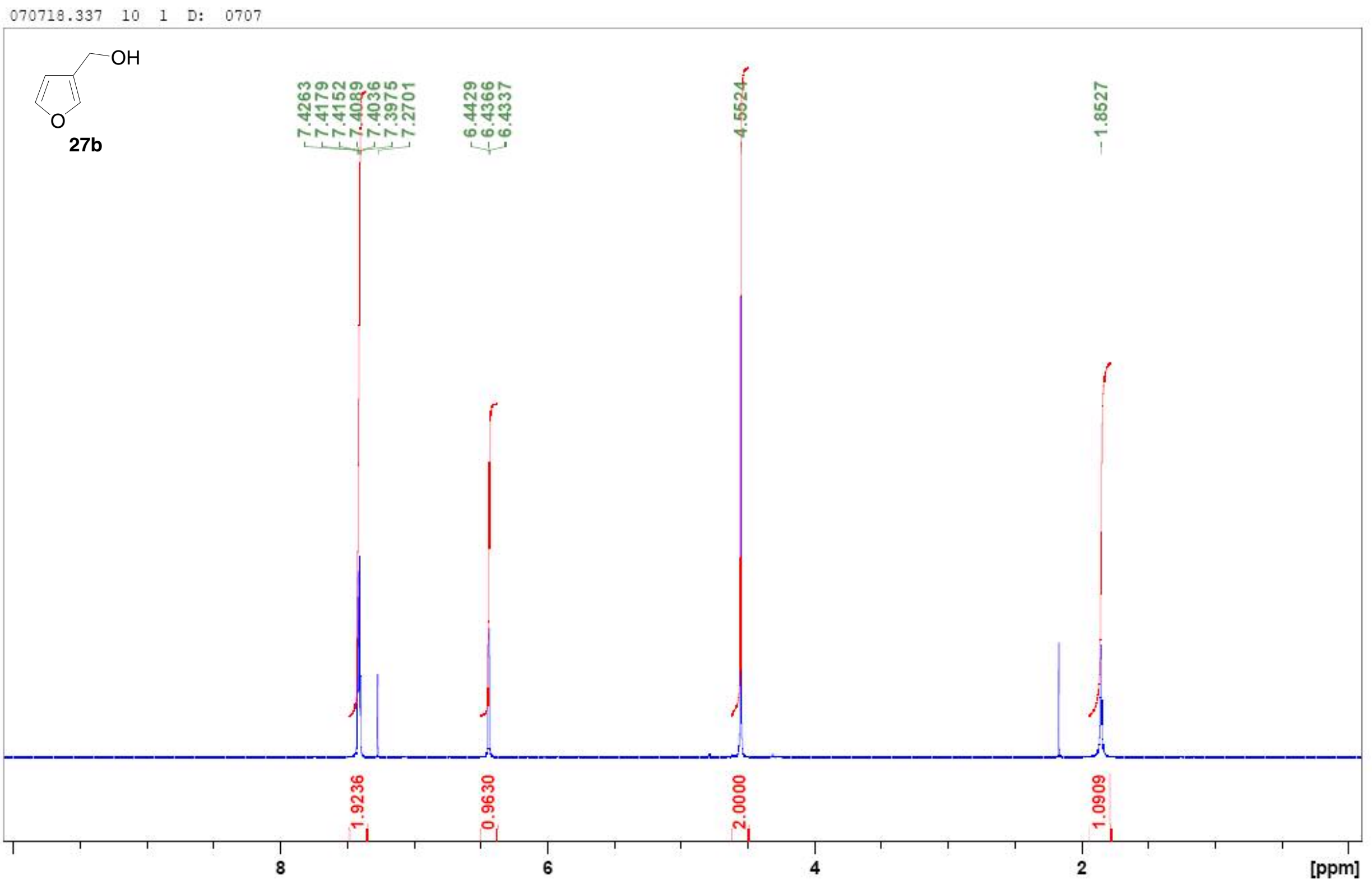


Supporting information-2

$070718.337 \quad 11 \quad 1 \quad$ D: 0707

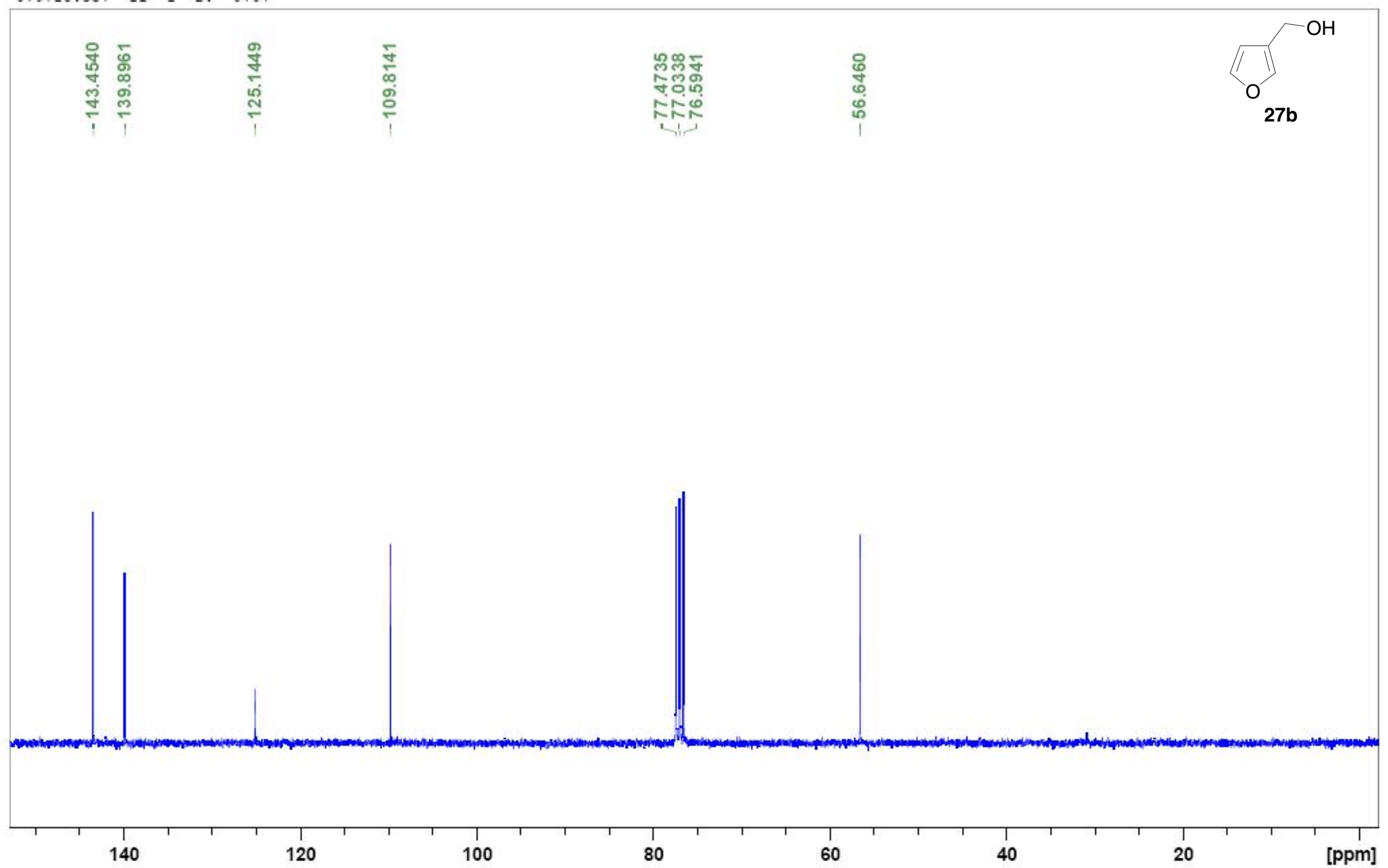


$070718.335 \quad 10 \quad 1 \quad$ D: 0707

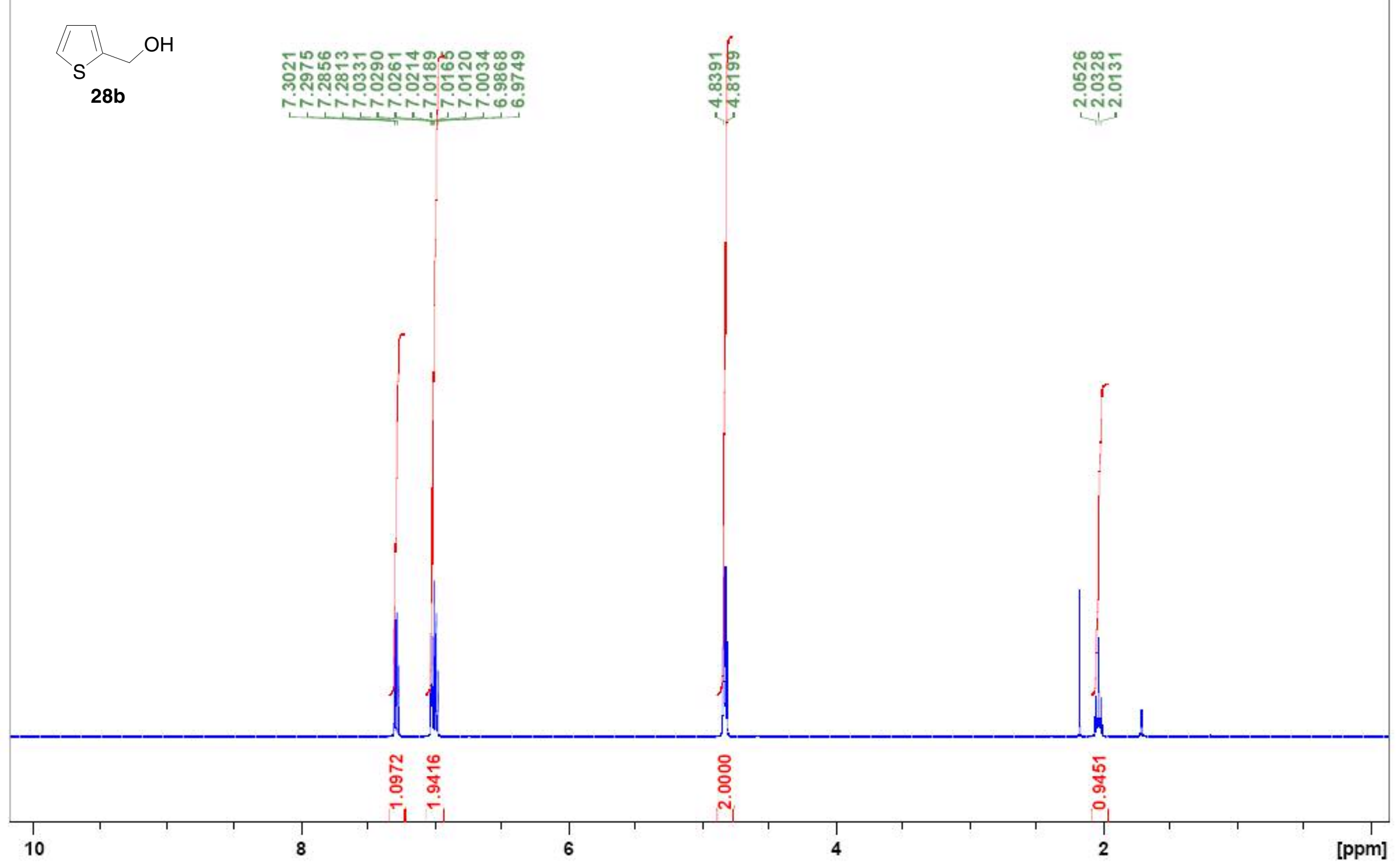


Supporting information-2

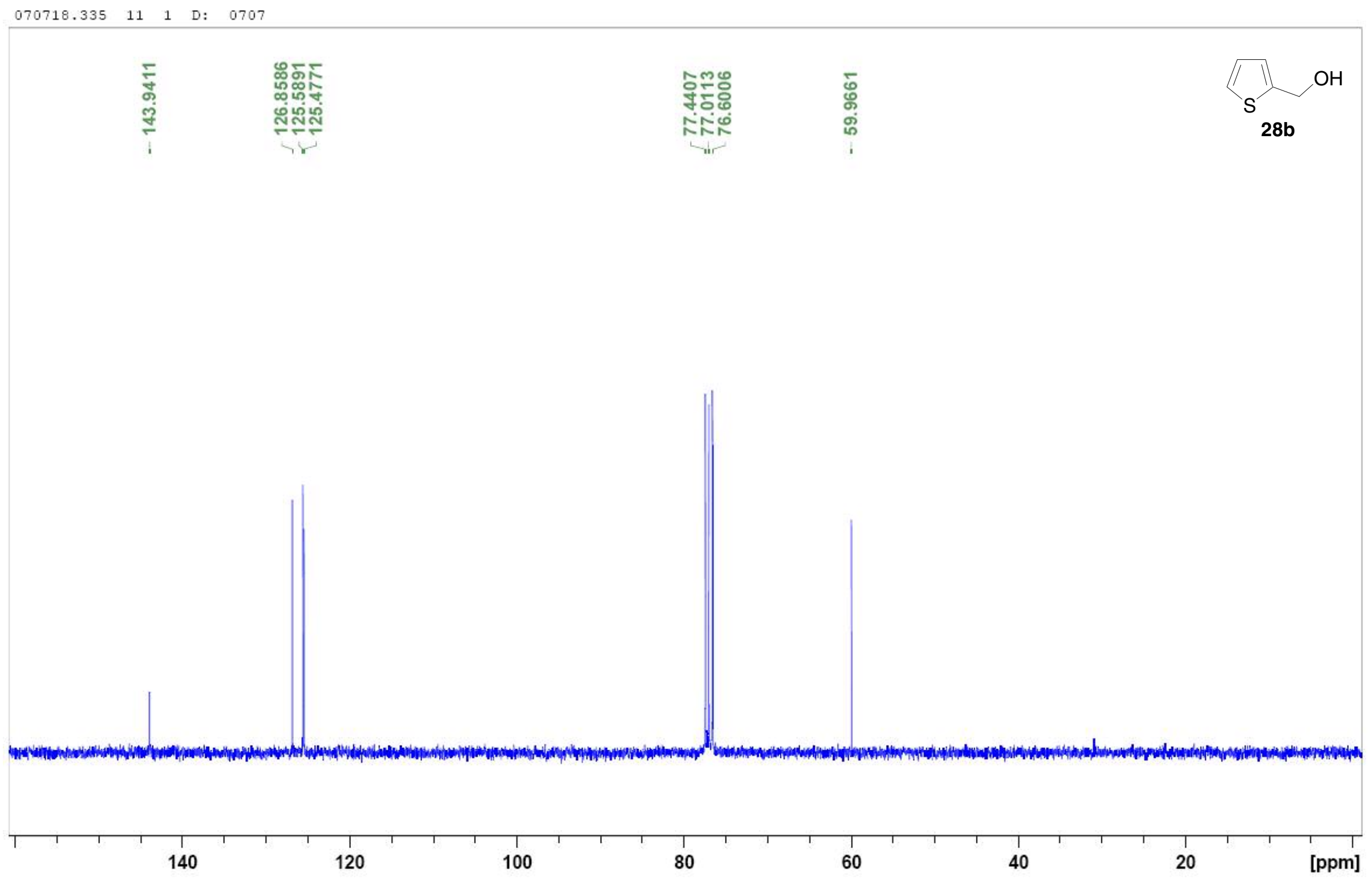


$070727.337 \quad 10 \quad 1 \quad$ D: 0707

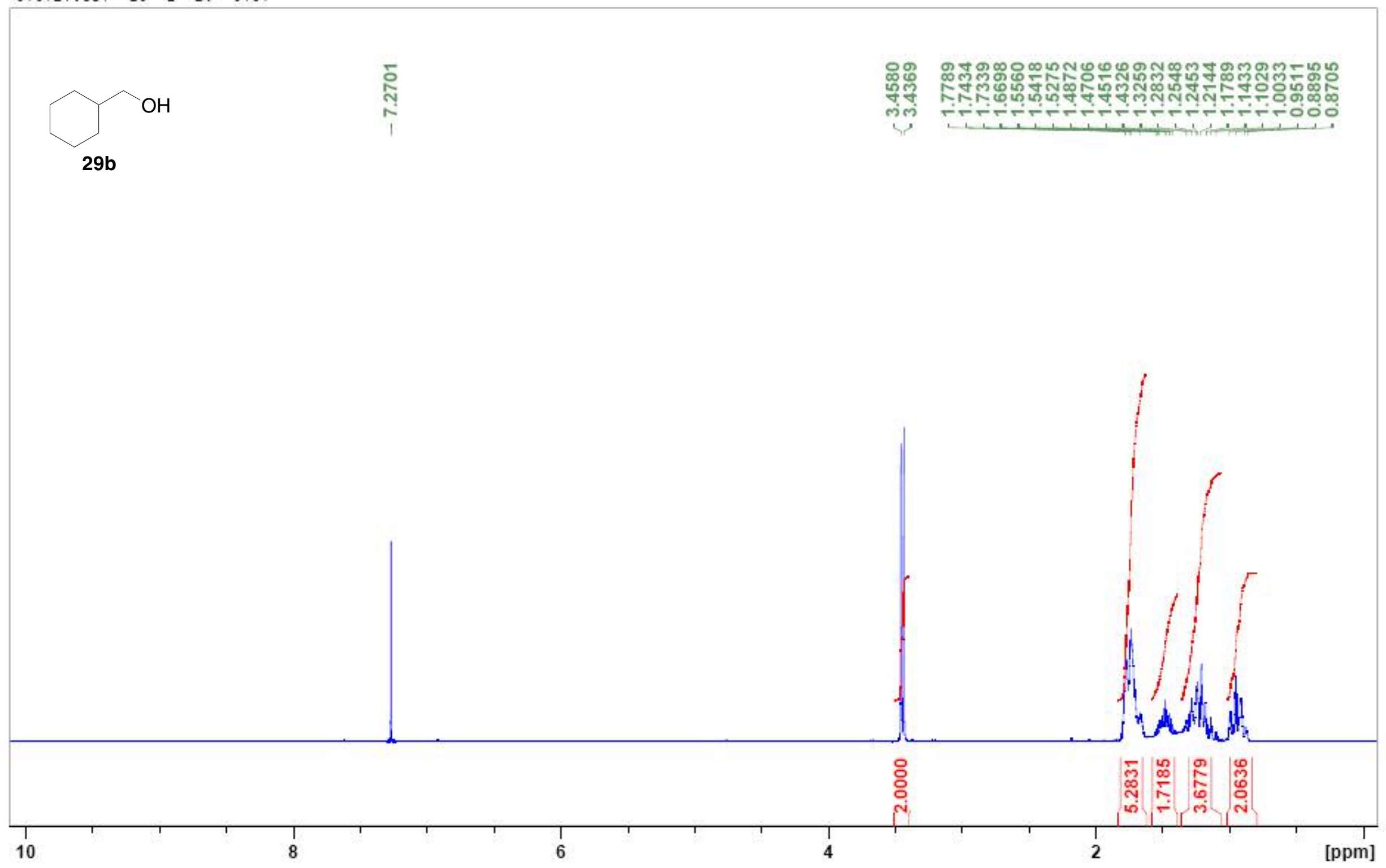


$070727.337 \quad 11 \quad 1 \quad$ D: 0707

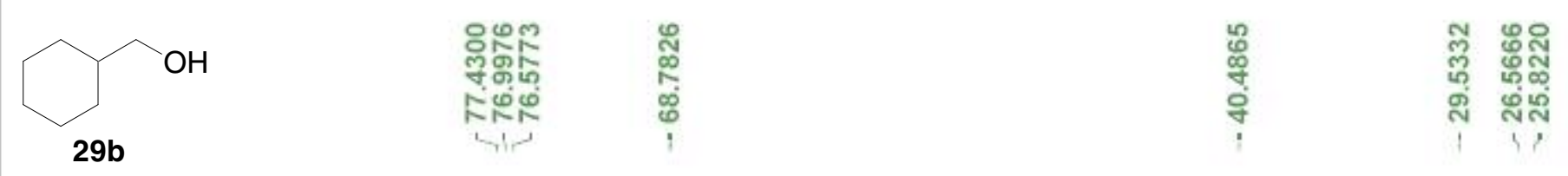

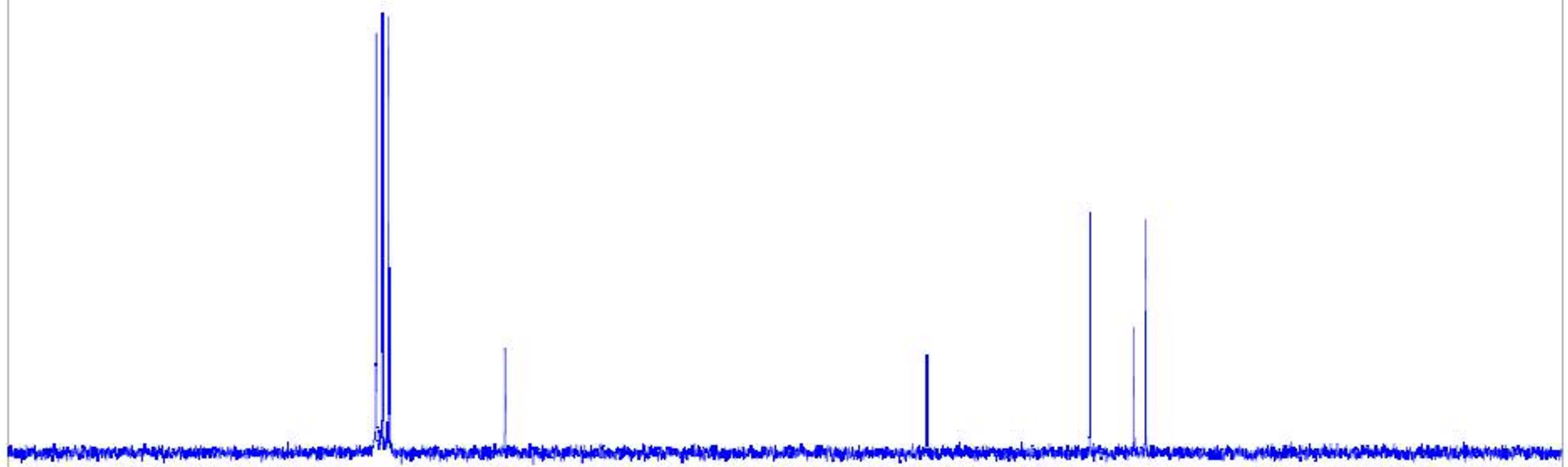


$070914.326 \quad 10 \quad 1 \quad$ D: 0709

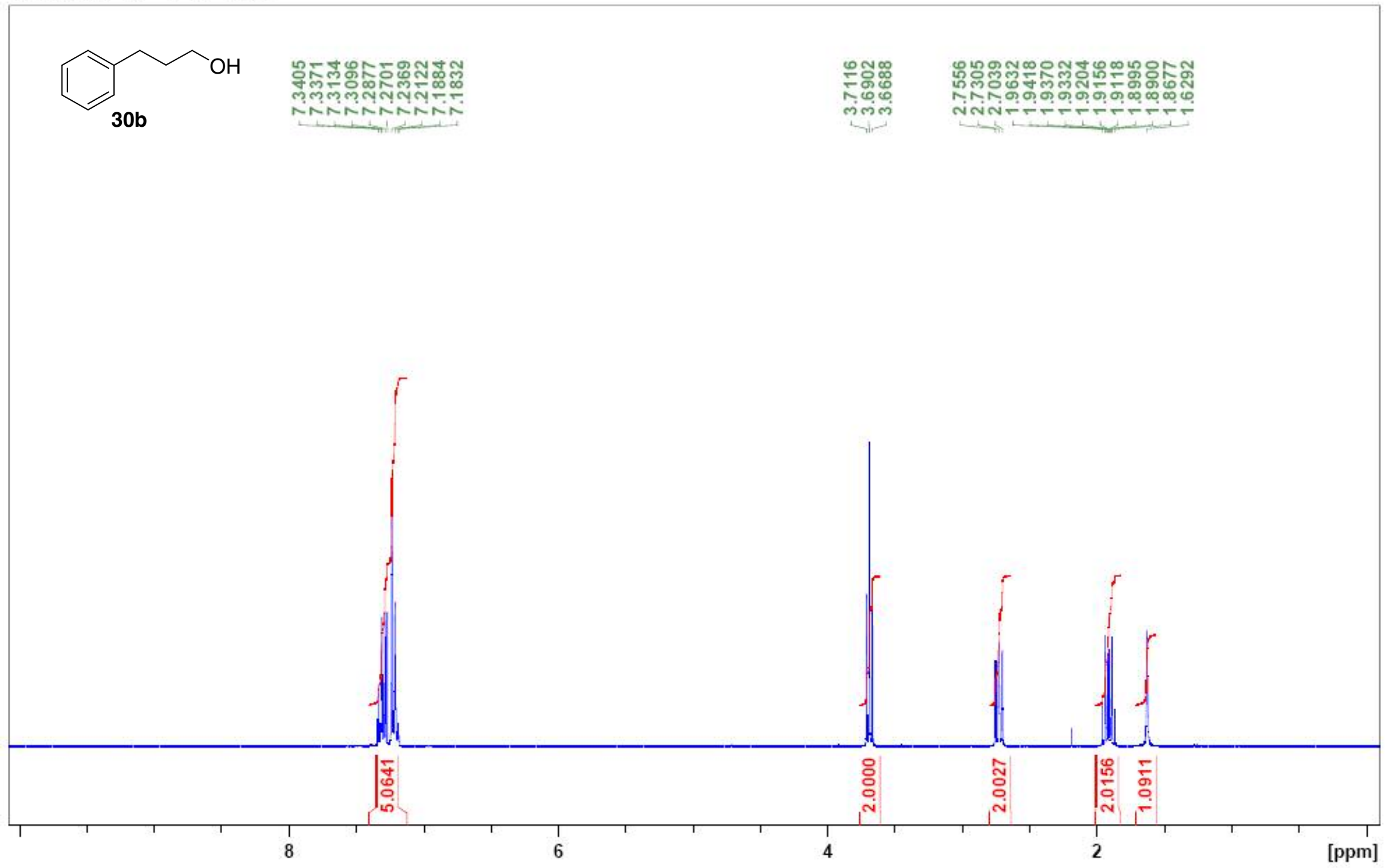


$070914.32611 \quad 1 \quad$ D: 0709

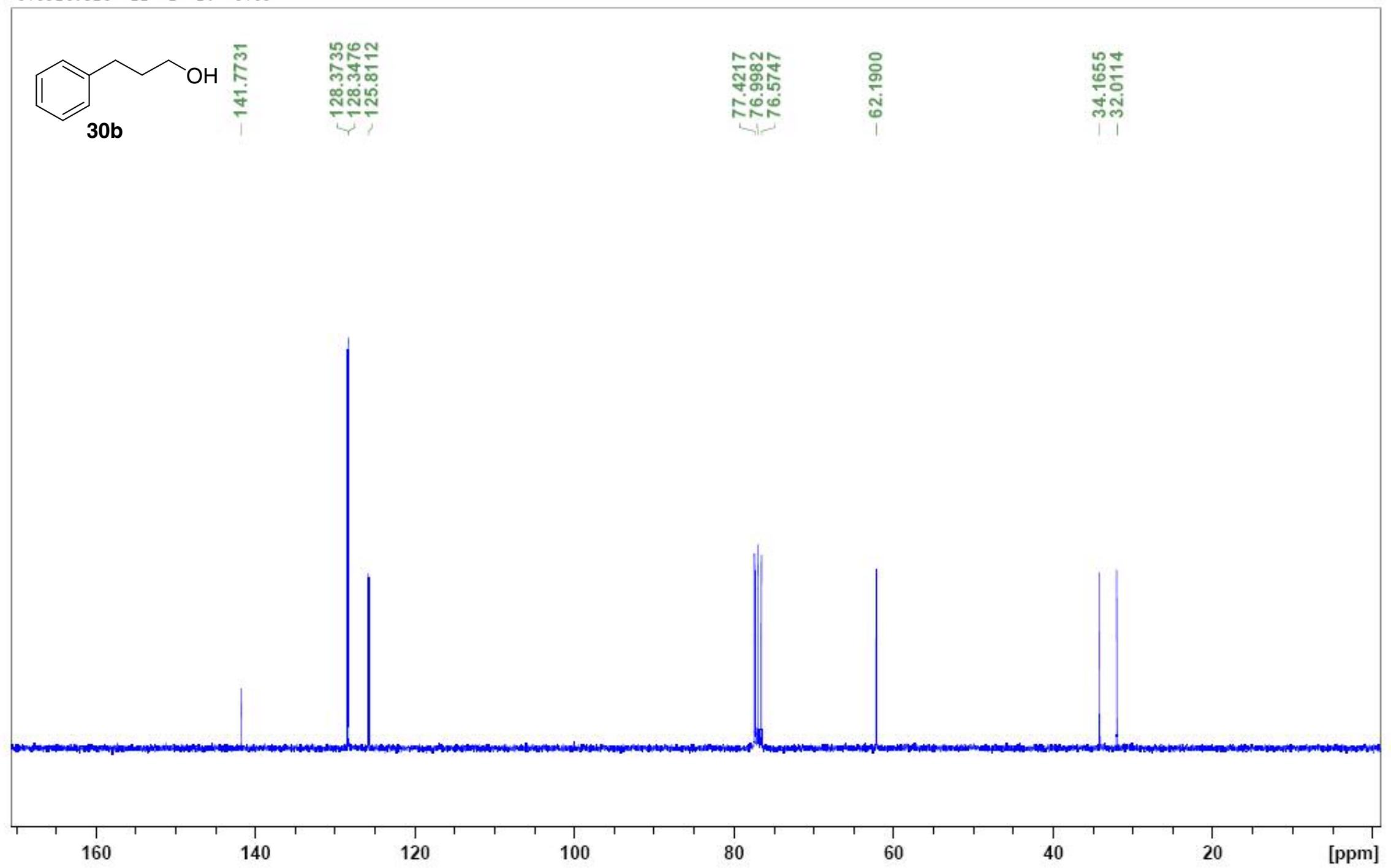




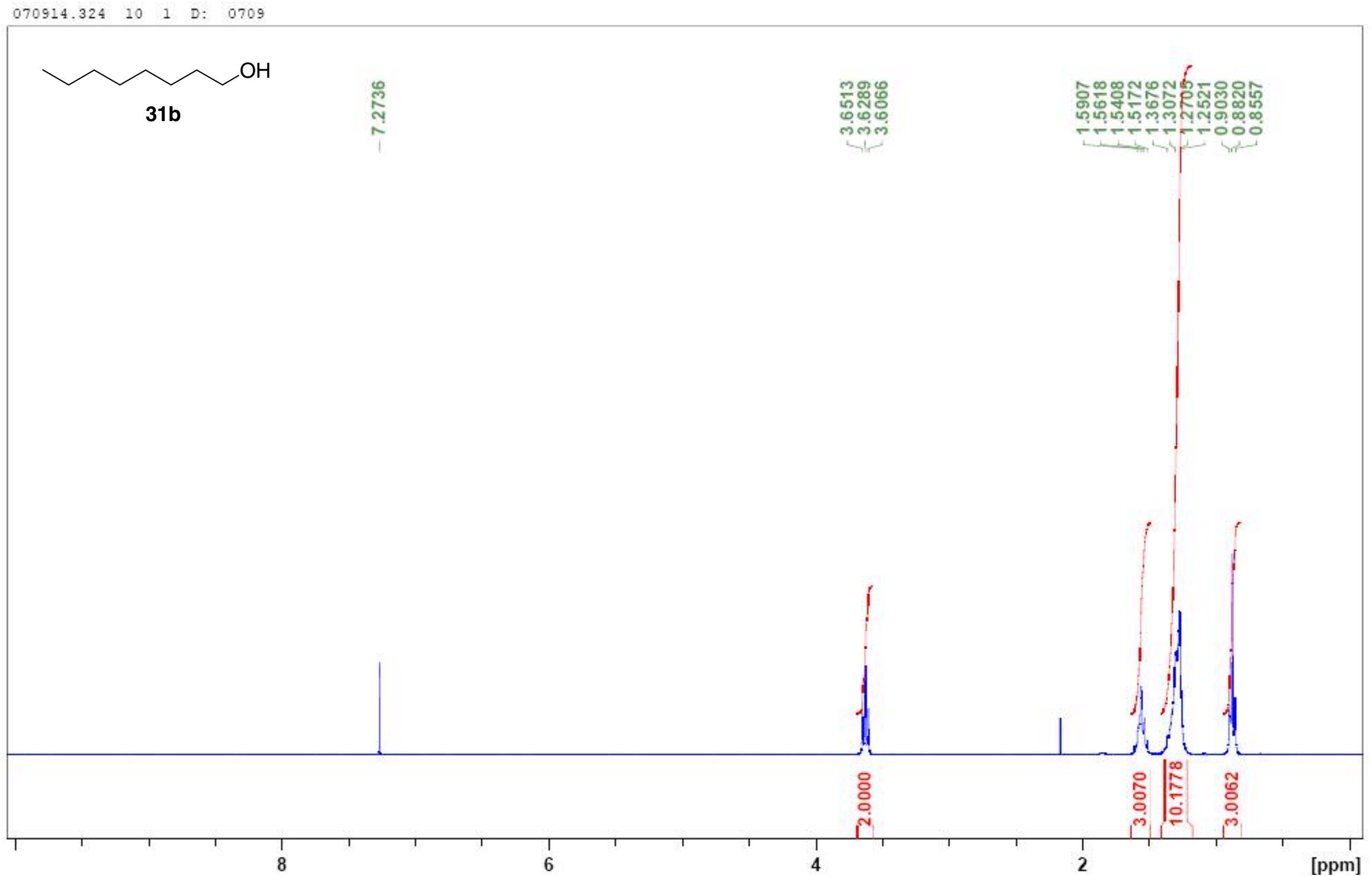


Supporting information-2

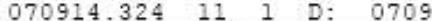

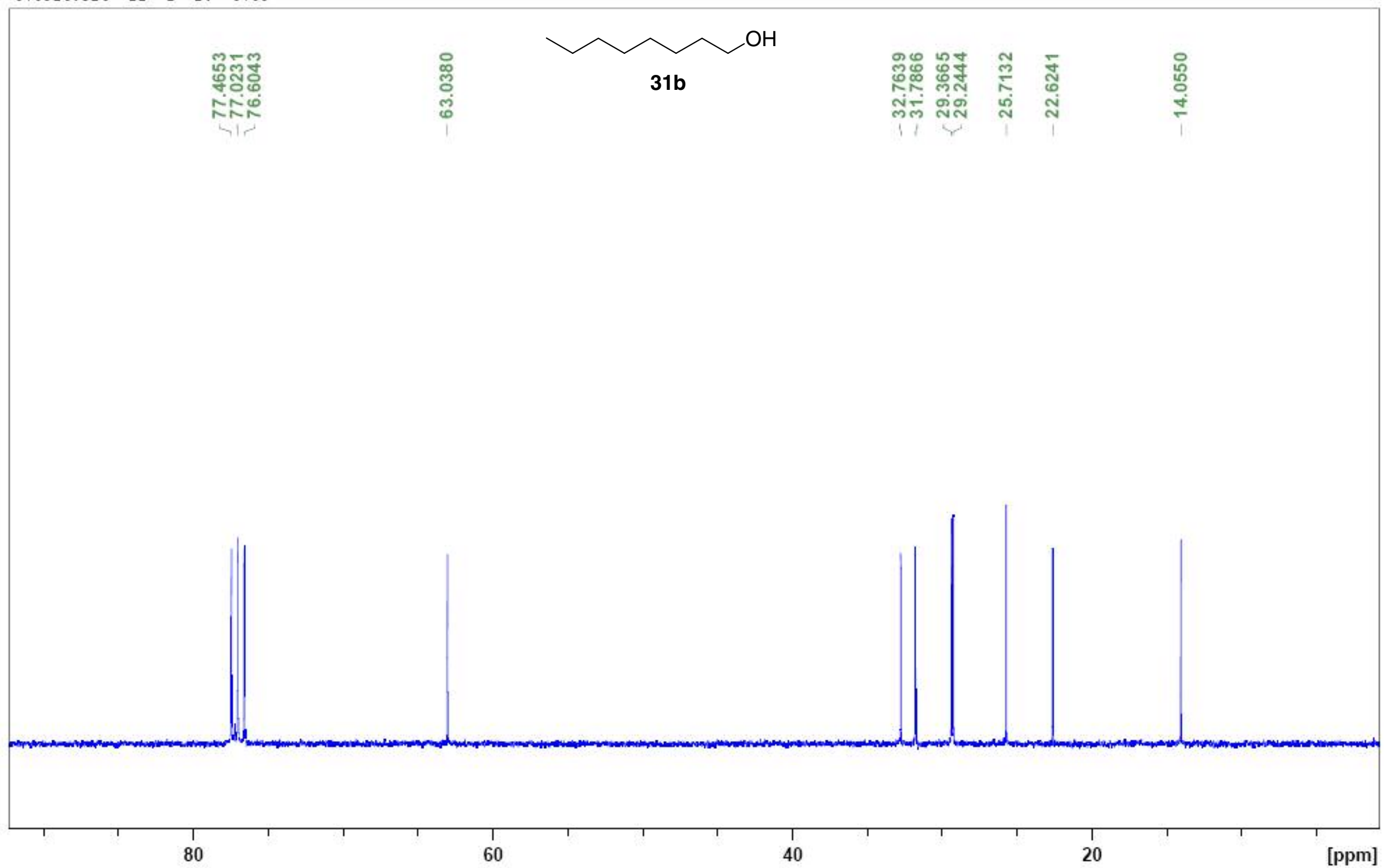


Supporting information-2

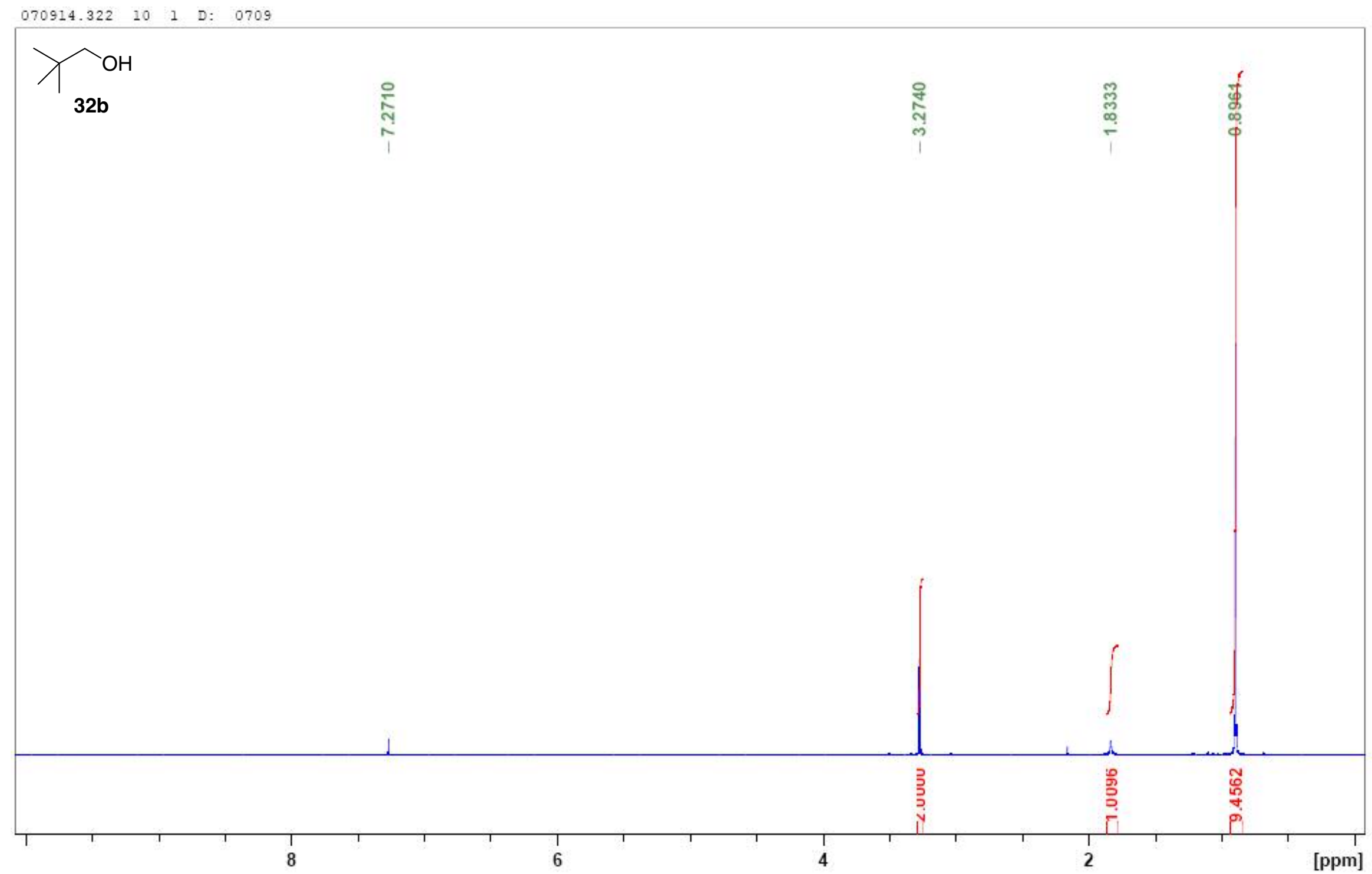


Supporting information-2

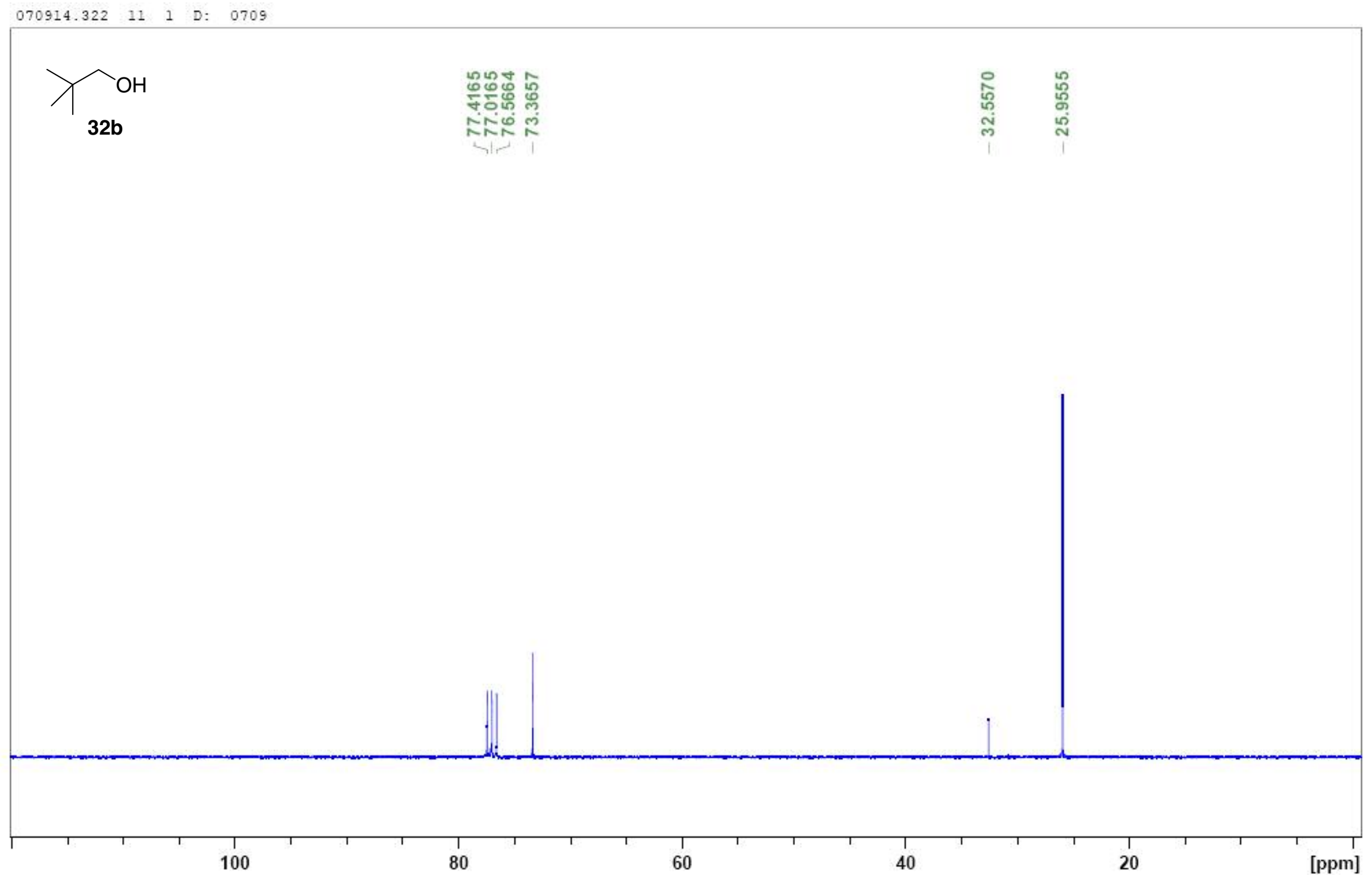




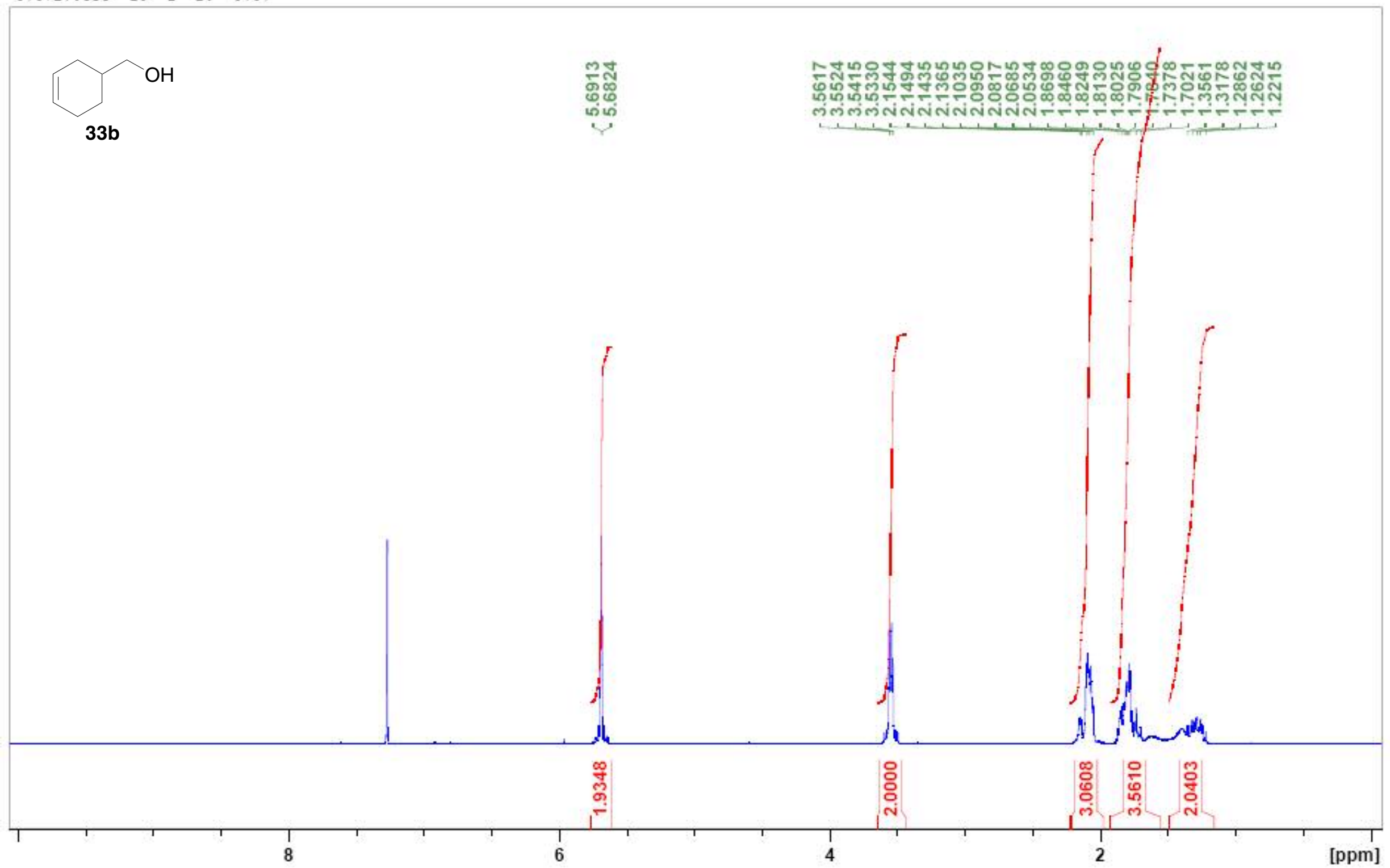




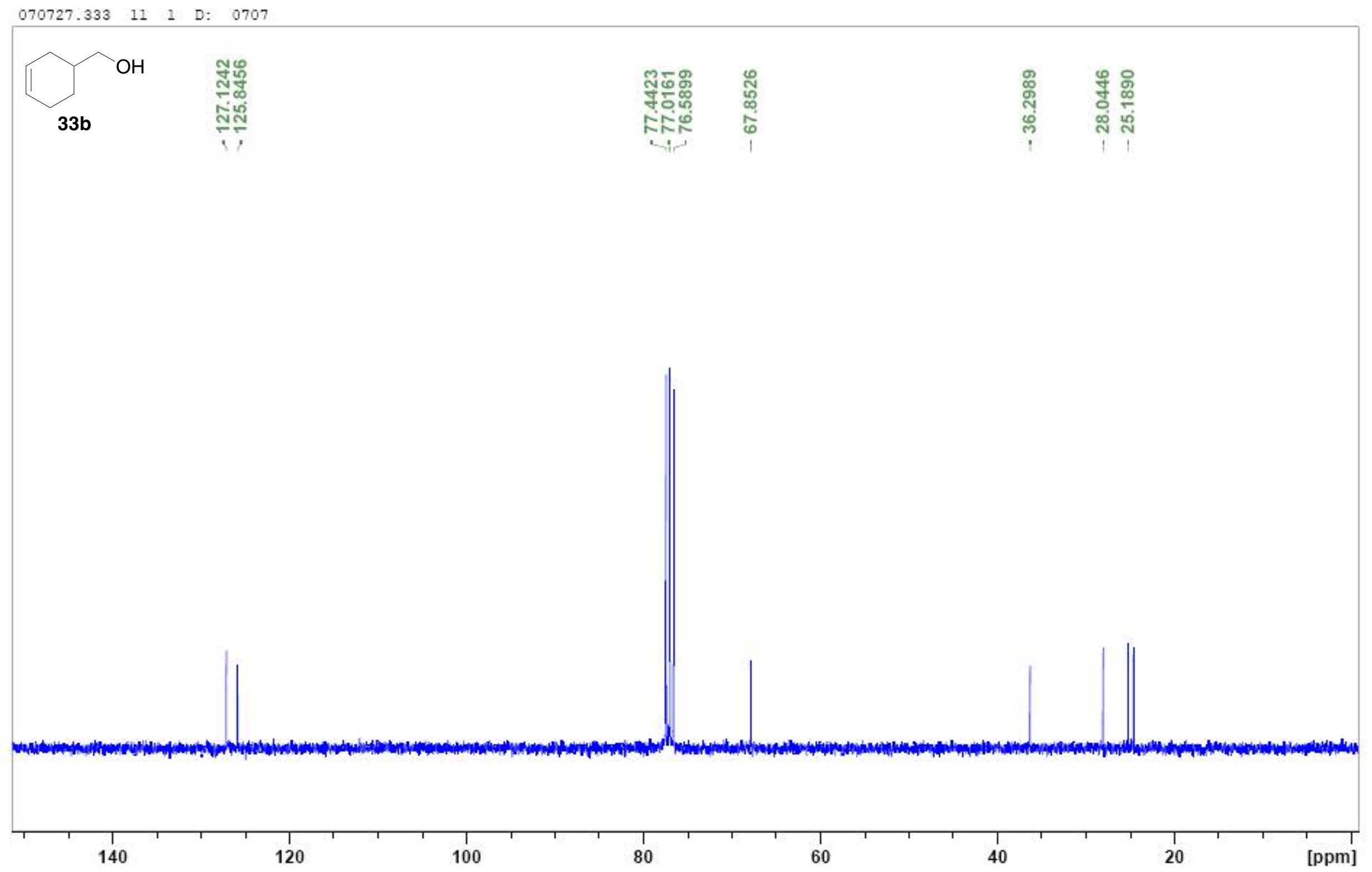




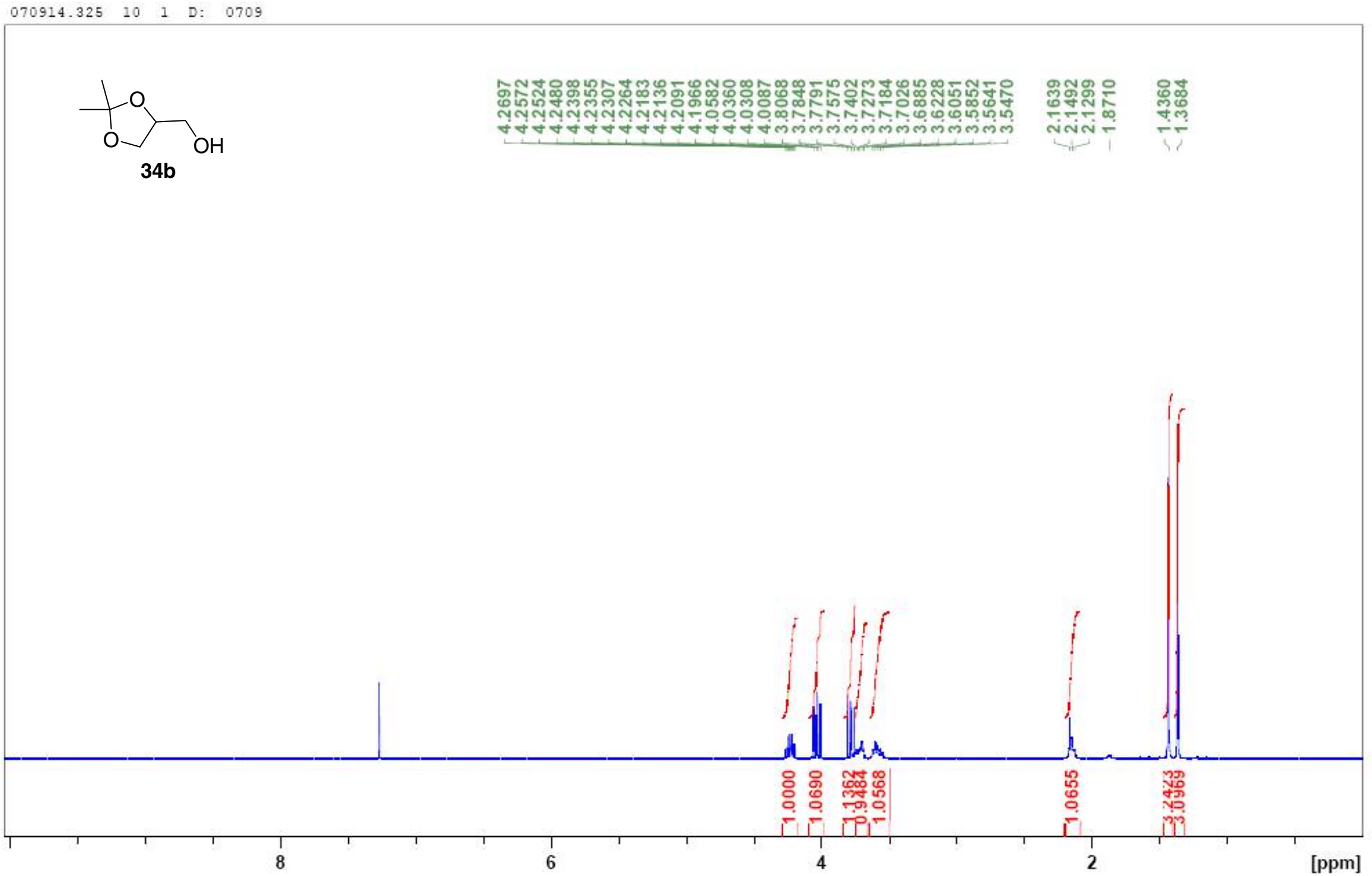




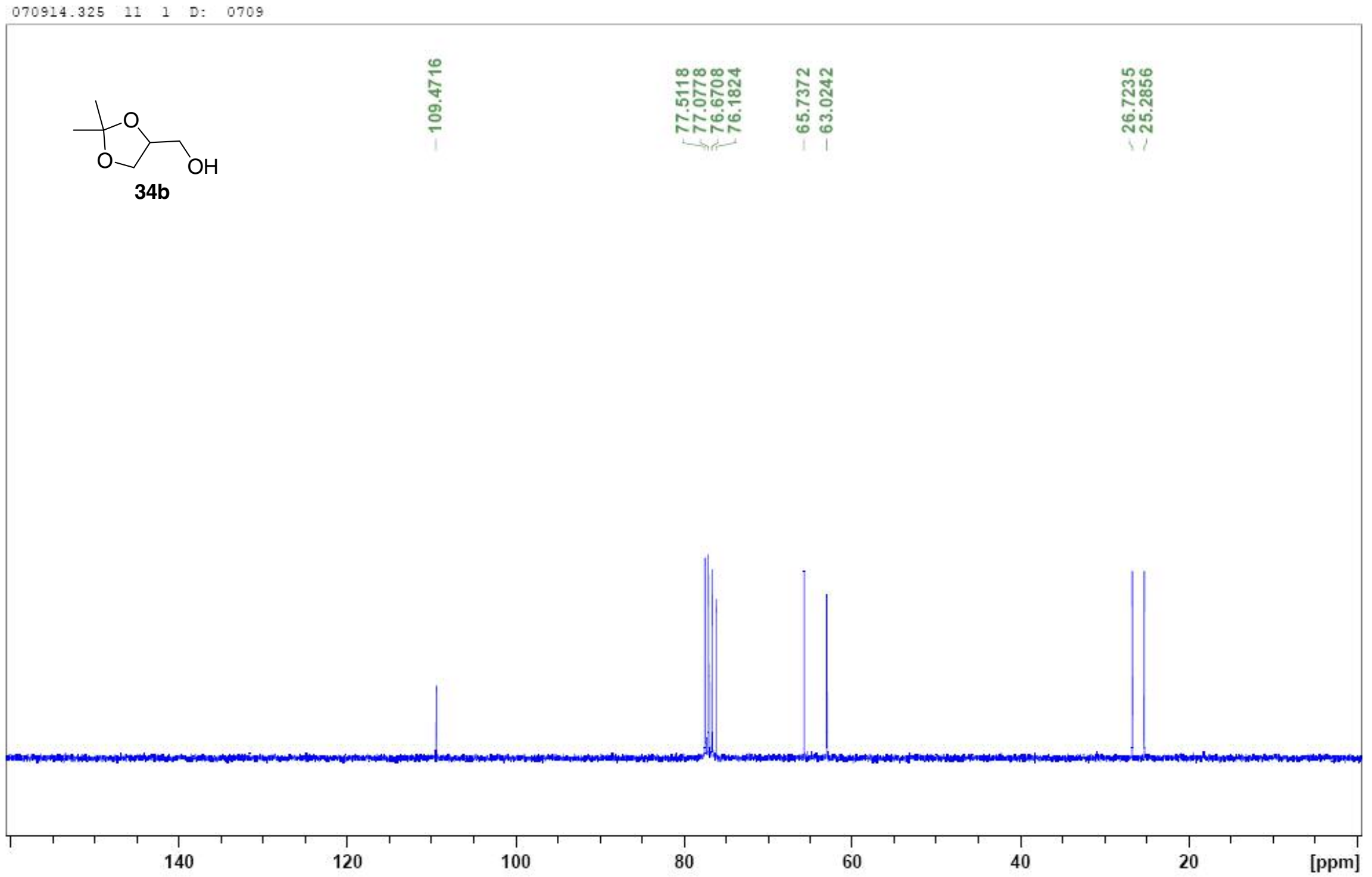


$070716.327 \quad 10 \quad 1 \quad$ D: $\quad 0707$

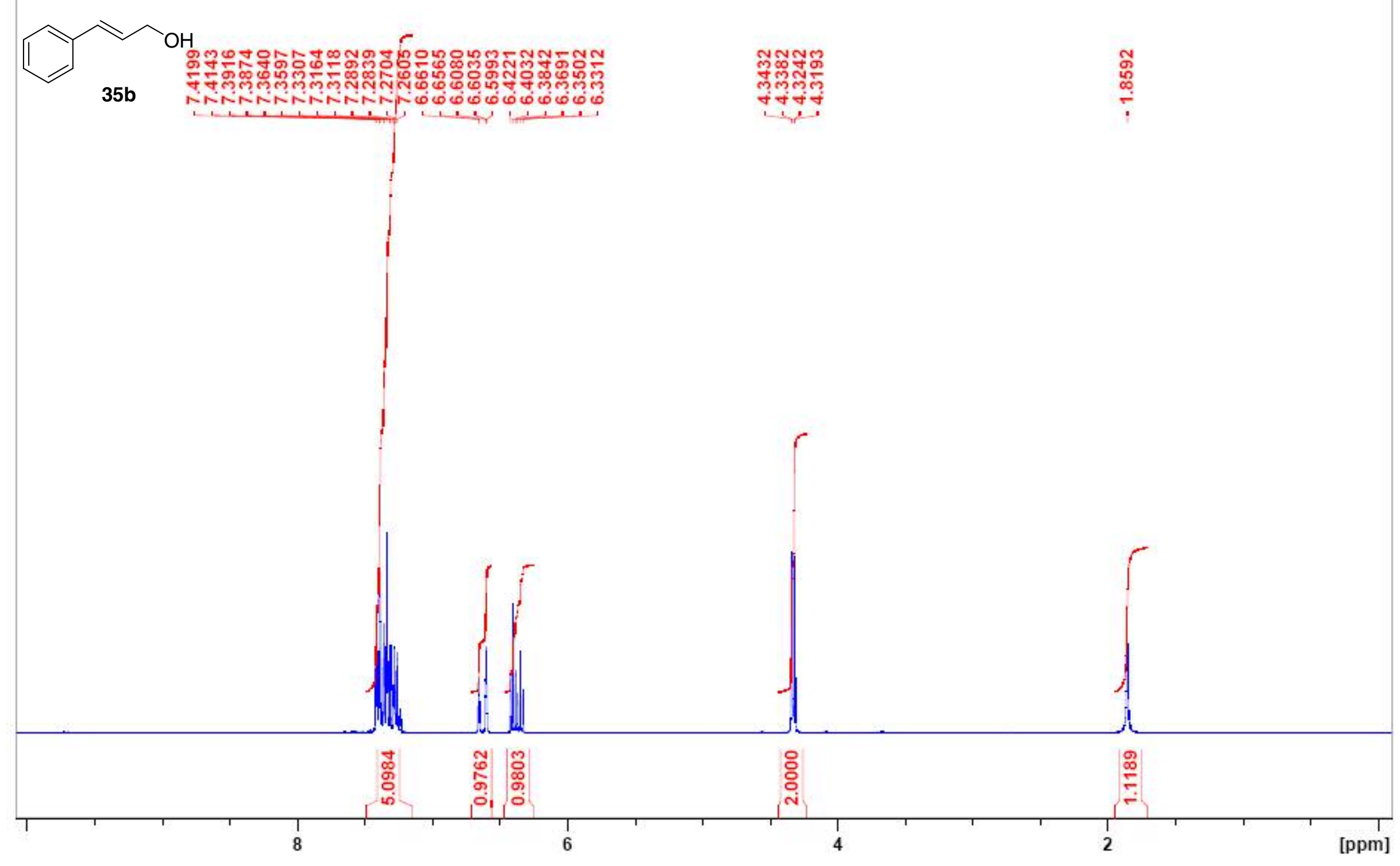




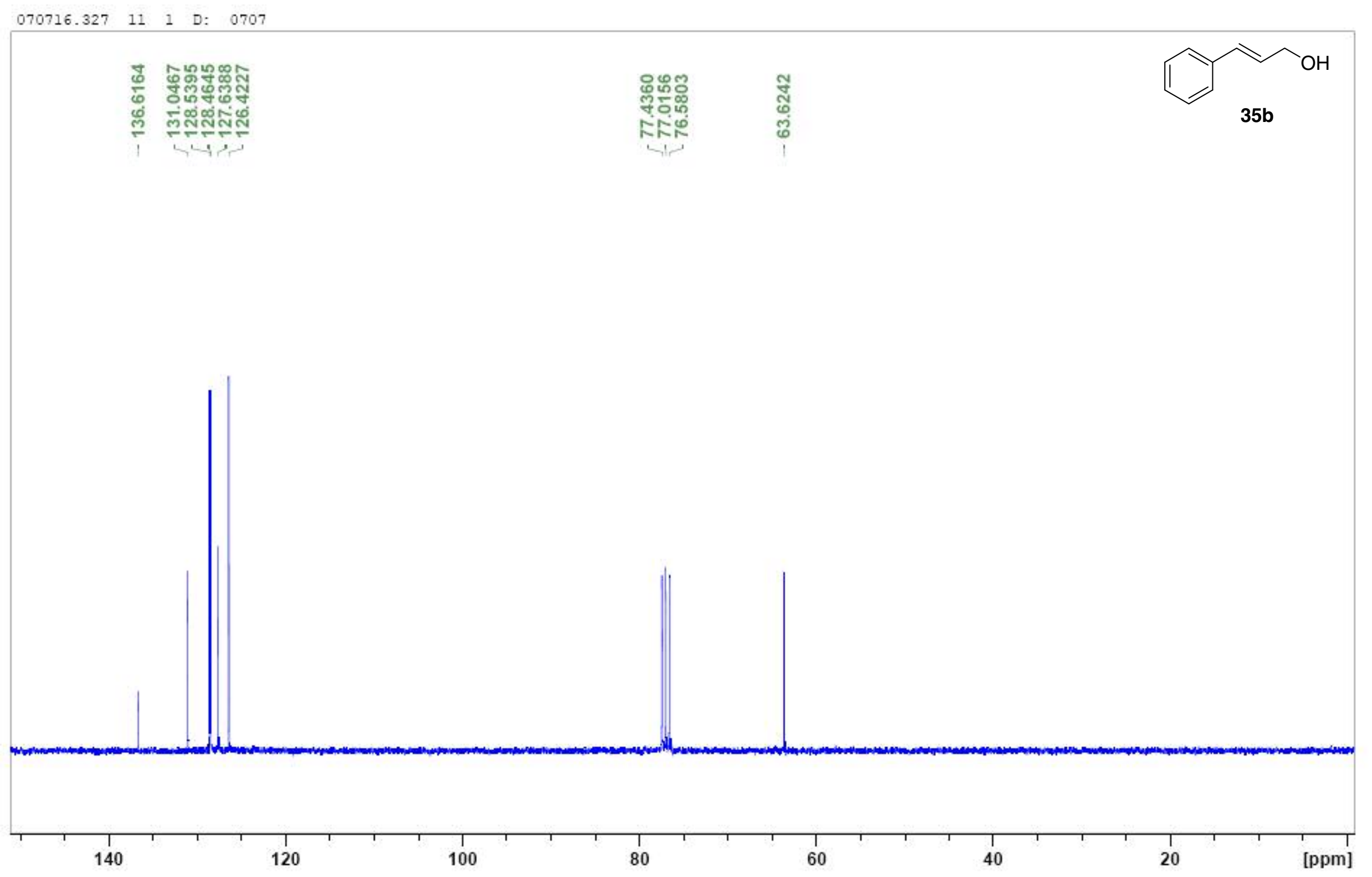

